\title{
Representation of the rewarding value of food in the humann brain - Effects of dietary restraint, body weight, stress, and shifts in macronutrient intake
}

Citation for published version (APA):

Born, J. M. (2011). Representation of the rewarding value of food in the humann brain - Effects of dietary restraint, body weight, stress, and shifts in macronutrient intake. [Doctoral Thesis, Maastricht University]. https://doi.org/10.26481/dis.20111208jb

Document status and date:

Published: 01/01/2011

DOI:

10.26481/dis.20111208jb

Document Version:

Publisher's PDF, also known as Version of record

Please check the document version of this publication:

- A submitted manuscript is the version of the article upon submission and before peer-review. There can be important differences between the submitted version and the official published version of record.

People interested in the research are advised to contact the author for the final version of the publication, or visit the DOI to the publisher's website.

- The final author version and the galley proof are versions of the publication after peer review.

- The final published version features the final layout of the paper including the volume, issue and page numbers.

Link to publication

\footnotetext{
General rights rights.

- You may freely distribute the URL identifying the publication in the public portal. please follow below link for the End User Agreement:

www.umlib.nl/taverne-license

Take down policy

If you believe that this document breaches copyright please contact us at:

repository@maastrichtuniversity.nl

providing details and we will investigate your claim.
}

Copyright and moral rights for the publications made accessible in the public portal are retained by the authors and/or other copyright owners and it is a condition of accessing publications that users recognise and abide by the legal requirements associated with these

- Users may download and print one copy of any publication from the public portal for the purpose of private study or research.

- You may not further distribute the material or use it for any profit-making activity or commercial gain

If the publication is distributed under the terms of Article 25fa of the Dutch Copyright Act, indicated by the "Taverne" license above, 


\section{Representation of the rewarding value of food in the human brain}

Effects of dietary restraint, body weight, stress, and shifts in macronutrient intake 
Financial support for the publication of this thesis was gratefully received from Valesta B.V.

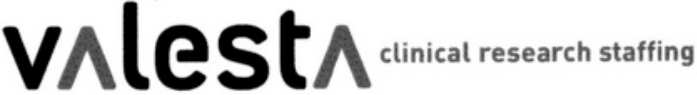 \\ www.valesta.com}
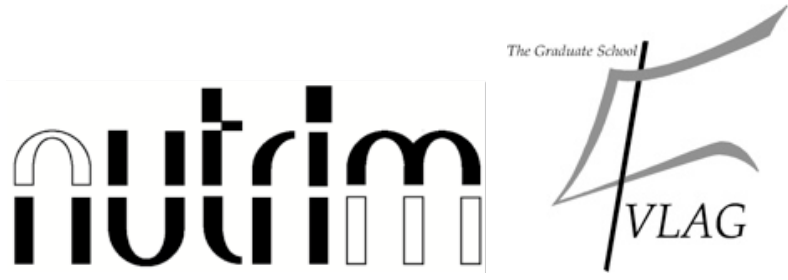

The study presented in this thesis was performed within NUTRIM School for Nutrition, Toxicology and Metabolism, which participater in the Graduate School VLAG (Food Techonology, Agrobiotechnology, Nutrition and Health Sciences), accredited by the Royal Netherlands Academy of Arts and Sciences

Cover design \& Layout: Jurriaan Born

Printed by Boxpress B.V.

(C) Copyright Jurriaan M. Born, Maastricht 2011 ISBN 978-90-8891-349-5 


\title{
Representation of the rewarding value of food in the human brain
}

\author{
Effects of dietary restraint, body weight, stress, \\ and shifts in macronutrient intake
}

\section{Proefschrift}

ter verkrijging van de graad van doctor aan de Universiteit Maastricht

op gezag van de Rector Magnificus

Prof. mr. G.P.M.F. Mols

volgens het besluit van het College van Decanen

in het openbaar te verdedigen

op donderdag 8 december 2011 om 14:00 uur

door

Jurriaan Martijn Born

Geboren te Herdecke op 19 mei 1978 


\section{Promotores:}

Prof. dr. M.S. Westerterp-Plantenga

Prof. dr. R.W. Goebel

\section{Beoordelingscommissie:}

Prof. dr. ir. R.P. Mensink (voorzitter)

Prof. dr. A.A.M. Masclee

Prof. dr. Timothy Moran (Johns Hopkins Univ. School of Medicine, USA)

Prof. dr. ir. A. Schols

Dr. A. Takashima (Donders Institute, Nijmegen) 


\section{Table of Contents}

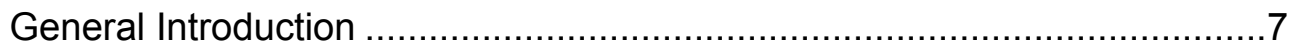

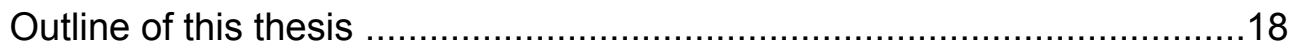

Differences between liking and wanting signals in the human brain and relations with cognitive dietary restraint and body mass index

A Nonlinear relationship between food choice related brain signaling in the absence of hunger and BMI

Acute stress and food-related reward activation in the brain during food choice during eating in the absence of hunger

High HPA-axis activation disrupts the link between liking and wanting with liking and wanting related brain signaling

Shifts in protein versus carbohydrate intake differentially affects liking and wanting related brain signaling

General Discussion

Summary

Samenvatting 105

Acknowledgements 109

List of Publications

About the Author 

Chapter 1

General Introduction 
In the developed world, the prevalence of obesity has been growing over the past decades, reaching epidemic proportions ${ }^{1,2,3}$. This has been receiving increasing attention, especially since visceral (or central) obesity plays a key role in the development of the metabolic syndrome, diabetes and ultimately cardiovascular disease ${ }^{2,4,5,6}$. The etiology of overweight and obesity has been attributed to numerous causes, including genetic, physiological, environmental and behavioral factors ${ }^{7,8,9}$. In this thesis, methods are explored to model brain activity using functional magnetic resonance imaging (fMRI) and the brain representation of the rewarding value of food. The effects of some cognitive and anthropometric factors, related to liking and wanting related signaling in the brain were studied. These factors included dietary restraint and disinhibition, and body mass, activation of the hypothalamus pituitary axis (HPA-axis) and shifts in macronutrient intake.

\section{Energy balance}

To maintain a constant supply of energy while maintaining stable body weight, energy intake and energy expenditure need to be in balance. A recent study observed that total energy expenditure did not decrease between 1988 and 2006. Thereby, it is suggested that the recent rise in obesity may result from maintaining positive energy balance through increased energy intake over long periods of time, rather than from a lack of physical exercise and the resulting lower activity induced energy expenditure ${ }^{10}$. This idea is supported by studies that have indicated that the increased incidence of obesity was paralleled with increasing portion sizes of commercially available foods and sweetened drinks and also possibly increased sugar and fat intake ${ }^{11,12}$.

In controlling energy intake with respect to energy balance, two modes have been described: Firstly, homeostatic eating, in which energy intake matches energy expenditure. Here, energy intake is related to hunger and satiety and this mode of eating leads to energy balance and a steady body weight. Secondly, nonhomeostatic eating, in which energy intake does not match energy expenditure, e.g. eating in the absence of hunger ${ }^{13}$. This non-homeostatic eating over extended periods of time, by definition leads to body-weight change, if the energy imbalance is not restored e.g. through adjusting energy expenditure by altering the physical activity level ${ }^{13}$. It has been shown that homeostatic eating does not necessarily take place on a daily basis: Subjects that were in energy balance had a highly variable energy intake from day to day, but over a week, energy intake was closely related to energy expenditure ${ }^{14}$, indicating that homeostatic regulation may take place over a number of days. In non-homeostatic eating, the degree to which overeating takes place has been attributed to the rewarding value of food ${ }^{7,15,16}$.

In the brain, homeostatic and non-homeostatic processes are attributed to two divergent pathways ${ }^{13}$. Homeostatic signaling is derived from two origins (Figure 1): Firstly, orosensory signals and sensory input from the gastrointestinal tract that arrive in the central nervous center through the nucleus of the solitary tract (NTS), from which they are projected through the parabrachial nucleus to the thalamus and the primary taste cortex ${ }^{15,17}$. Additional sensory input, namely olfactory signals, originate in the olfactory bulb, and are projected to the olfactory cortex, converging with the taste signals in the hypothalamus and orbitofrontal cortex ${ }^{18}$. Secondly, the 
energy state is sensed through several pathways: Food intake leads to uptake of nutrients into the blood stream (glucose, amino acids, free fatty acids) and to gastrointestinal release of numerous regulatory peptide hormones, such as insulin, leptin, cholecystokinin (CCK), peptide YY (PYY), glucagon-like peptide 1 (GLP1) and to the inhibition of the ghrelin ${ }^{18,19}$. Signaling of the energy state follows two distinct routes. The first route, is signaling through the vagus nerve, which connects the peripheral organs to the brain. Signals that arrive in the NTS from the vagus nerve include gastric distention signals and signals derived from receptors for CCK, leptin, GLP1 and PYY ${ }^{20,21}$ and these hormonal receptors on the vagal afferents are

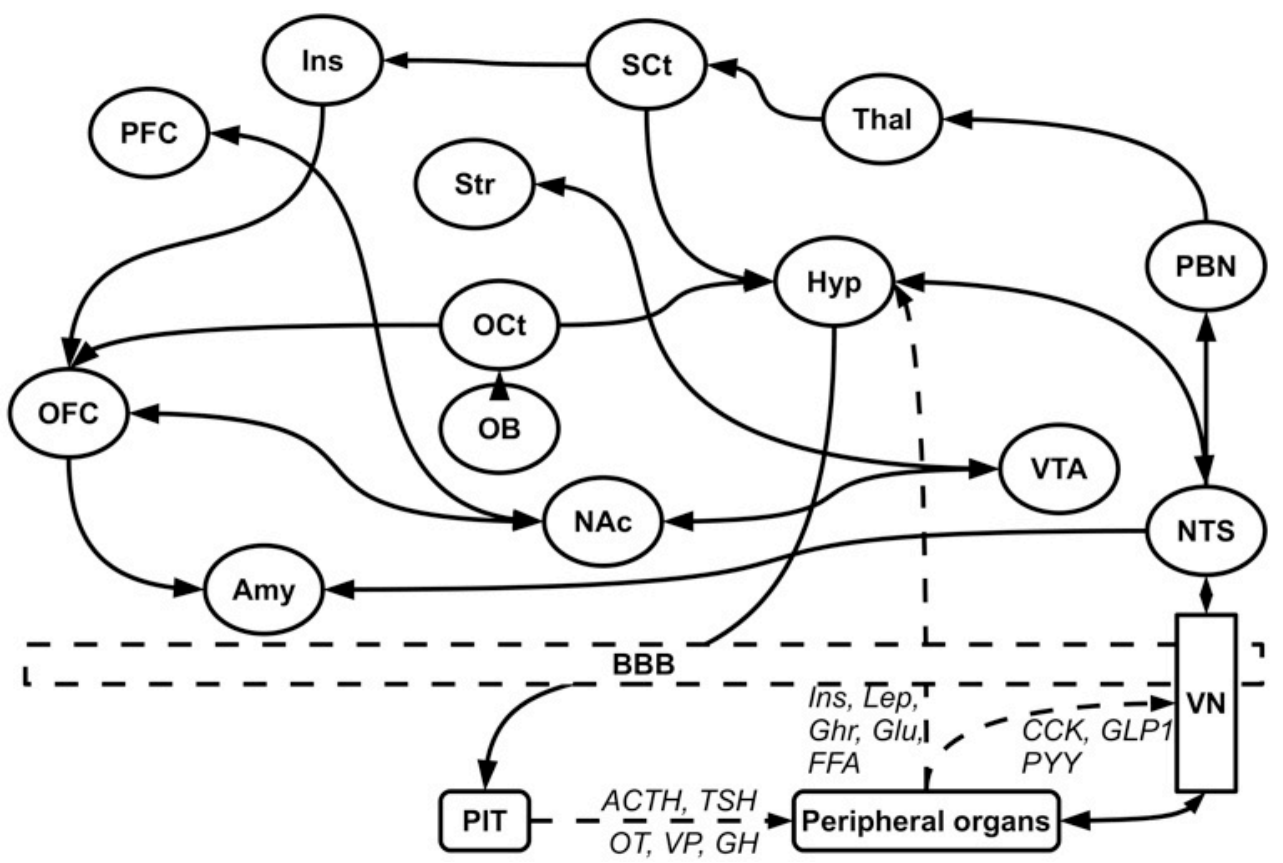

Figure 1: The homeostatic system.

Nerve inputs from peripheral organs, including the gastrointestinal tract, are received in the nucleus of the solitary tract (NTS) through the vagus nerve (nervus vagus, NV). These Gastrointestinal and taste signals received in the NTS are signaled to the hypothalamus (Hyp) and to the parabrachial nucleus (PBN), thalamus (Thal), sensory cortex (SCt) and further through the insula (Ins) to the orbitofrontal cortex (OFC). Olfactory input from the olfactory bulb (OB) is signaled to the hypothalamus and OFC. Another connection leads from the NTS to the Amygdala (Amy), which also receives input from the OFC. Loops between the OFC, nucleus accumbens (NAc), ventral tegmental area (VTA), striatum (Str) and prefrontal cortex (PFC), connect the homeostatic processes to the motivational system, which is required to execute homeostatic eating. In the hypothalamus, additionally receptors for nutrients and peptide hormones are expressed. These substances enter the brain through the blood brain barrier (BBB). Regulatory output from the hypothalamus targets the NTS and the periphery through the NV, as well as neuronal projections to the pituitary gland (PIT), thereby regulating release of multiple hormones into the blood stream. Hormones are indicated in italics and hormone signaling is indicated with an intermittent line. PFC: prefrontal cortex, CCK: Cholecystekinin, GLP1: glucagon-like peptide 1, PYY: peptide YY, Ins: insulin, Lep: leptin, Ghr: ghrelin, Glu: Glucose, FFA: free fatty acids, ACTH: adrenocorticotrope hormone, TSH: thyroid stimulating hormone, OT: oxytocin, VP: vasopressin, GH: growth horomone. 
additionally modulated by peripheral glucose levels ${ }^{20}$. The NTS is connected to the ARC and PVN through neuroepinephrine and neuropeptide $Y$ (NPY) neurons ${ }^{22}$. In addition, nutrients and peptide hormones arrive in the brain through the blood brain barrier (BBB) and the transport of peptide hormones, such as insulin, leptin and ghrelin, involves active processes. These processes show diurnal variation, and modulation by other factors, e.g. the presence of insulin enhances leptin transport and triglycerides promote insulin transport, while inhibiting leptin and ghrelin transport ${ }^{19,23}$. In the brain, nutrient and peptide hormone sensing is predominantly located in the arcuate (ARC) and paraventricular nucleus (PVN), as well as the ventromedial $(\mathrm{VMH})$ part of the hypothalamus ${ }^{15,18,22,24,25}$. In the $A R C$, receptors for insulin, leptin and ghrelin have been found 15,18,25, there are indications for GLP1 signaling in the PVN and neurons sensitive to glucose and fatty acids and possibly amino acids have been discovered in the lateral hypothalamus ${ }^{18,24}$. Projections from the ARC to the LHA and finally NTS involve signaling with NPY and agouti-related protein (AgRP) neurons that stimulate food intake, as well as signaling by pro-opiomelanocortin (POMC)/a-melanocyte stimulating hormone ( $\mathrm{aMSH}$ )/cocaine and amphetamine regulated transcript (CART) neurons, which inhibit food intake ${ }^{18,22}$.

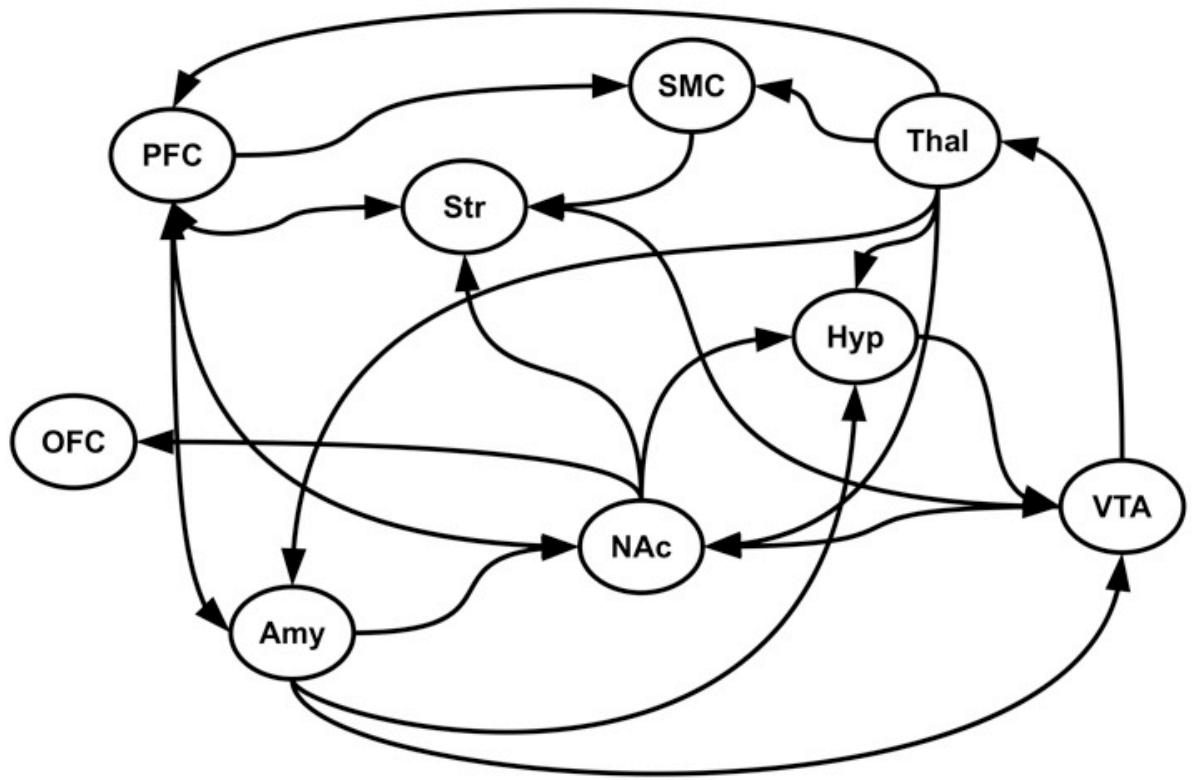

Figure 2: The motivational system.

This system is involved in non-homeostatic aspects of food intake. Key regions involved in motivational signaling are the regions of the dopamine mediated reward signaling, which are the ventral tegmental area (VTA), the striatum (Str) which includes the pallidum, putamen and caudate, and the nucleus accumbens (NAC). In return, the VTA receives returning input from the Str and NAc, and additionally from the hypothalamus (Hyp) and the amygdala (Amy). Some projections from the NAc target the orbitofrontal cortex (OFC), possibly modulating the hedonic properties associated with the current reward. The NAc, Amy and Str, reciprocally link to the prefrontal cortex (PFC), the location of executive functions, which receives further input from the thalamus. The PFC modulates the sensory signals that arrive from the thalamus (Thal) to the Str, in the sensory-motor cortex (SMC), where motor signals may also be induced resulting in behavior. 
The leptin induced signaling via the $\mathrm{POMC/aMSH}$ and its receptor, the melanocortin 4 receptor (MC4R), has become known as the leptin melanocortin pathway, and specifically this signaling cascade has been implicated strongly in feeding behavior, such that increased leptin leads to increased $\mathrm{aMSH}$ and MC4R activation, leading to decreased energy intake ${ }^{26}$. Signaling from the LHA, using melanocortin concentrating hormone $(\mathrm{MCH})$ and orexin, has been implicated in increasing food intake, while signaling from the PVN to the pituitary and NTS, involving corticotropin releasing hormone and oxytocin reportedly inhibits feeding behavior 18 . Experimentally, the importance of the hypothalamus in feeding behavior has been underscored in studies describing increased food intake after stimulating the lateral hypothalamus, while food intake seized when this region was damaged by lesions ${ }^{27,28}$. Additionally, in overweight and obese subjects, melanocortin signaling was specifically targeted with MC4R agonists and this has been shown to reduce food intake ${ }^{26,29}$. Overall, in this homeostatic system, the key areas are those associated with taste and hedonics, i.e. the orbitofrontal cortex and insula, and the hypothalamus as key recipient of peripheral input and source for peripheral signal output. Furthermore, parallel projections from the NTS and projections from the orbitofrontal cortex converge in the amygdala and nucleus accumbens (NAc), the latter of which has especially been implicated in the motivational aspects of eating behavior ${ }^{18,30}$.

Non-homeostatic signaling is largely independent of the nutritional status and is closely related to the rewarding value of food ${ }^{18,30,31}$. In this pathway the dopamine mediated reward circuitry is essential (Figure 2). Dopamine projections originates in the ventral tegmental area (VTA) and project to the NAc, striatum (caudate, putamen, globus pallidus) and the thalamus ${ }^{32,33,34}$. These areas connect to several other areas: From the NAc, projections loop back to the VTA and forward from the NAc to the prefrontal cortex, orbitofrontal cortex ${ }^{13,18}$, striatum and the amygdala; additional projections from the latter two return to the VTA ${ }^{33}$. Specifically the dopaminergic projections from the VTA to the NAc have been implicated in food intake: Direct stimulation in the NAc using dopamine increased food intake in rodents and cats ${ }^{35,36,37}$, and certain antipsychotics with an antagonistic effect on dopamine D2 receptors have been shown to significantly increase body weight in psychiatric patients ${ }^{38}$, indicating a key role for specifically dopamine in food intake regulation. Additional connections from the VTA to the thalamus, hypothalamus and returning to the VTA, modulate the VTA ${ }^{32}$ and loop between the prefrontal cortex and respectively the striatum, NAc and amygdala. These connections link the reward and taste systems to the executive functions of the prefrontal cortex 32,33,39,40. Overall, key areas in this non-homeostatic system are the striatum with input from the VTA, as well as the NAc and the amygdala. Connections from the prefrontal cortex to the sensory-motor cortex, may represent modulation of sensory signals and motor signals resulting in directed behavior, such as eating.

Behaviorally, non-homeostatic eating may be countered using cognitive dietary restraint. This process, together with disinhibition, i.e. loss of control and perceived feelings of hunger, are assessed using the 'Three Factor Eating Questionnaire' by Stunkard and Messinck ${ }^{41}$, and they each are represented by one of the factors (F1, F2 and F3, respectively ${ }^{41}$ ). Together, the three factors indicate the individuals' success in restricting energy-intake: successful dietary restraint is associated with relatively high scores for dietary restraint (TFEQ F1) and low disinhibition and 
feelings of hunger scores (TFEQ F2 and F3, respectively ${ }^{42,43}$ ). Conversely, unsuccessful dietary restraint is a consequence of high disinhibition and high feelings of hunger that may lead to the break down of restraint and increasing energy-intake $e^{43,44}$. While cognitive dietary restraint, disinhibition and feelings of hunger have been studied in behavioral studies, the question remained how the underlying processes may be represented in the brain.

\section{The rewarding value of food as liking and wanting}

Berridge proposed the 'incentive salience' theory 30 that divides the rewarding value of food into two distinct processes: 'Liking' which is the hedonic preference for, or palatability of each respective food-item. This liking is directly related to the taste and the pleasantness of taste that is associated with the respective food item. 'Wanting' is the motivation to eat a particular food item, which is related to the reinforcing value and reward that is associated with the respective item ${ }^{30}$. Liking and wanting may occur in synchrony, however, although there are clear indications that liking and wanting are related, they are not the same process: individuals may like something without acutely wanting it, such as liking a food while satiated, or wanting something without necessarily liking it, which is especially true for drugs of abuse $^{30,45}$. Additionally, this may apply to meal-specific food items. The combination of liking and wanting defines to a large extent the rewarding value of a given food item and thereby its specific perceived food reward 30 . Ultimately, it is argued that energy-intake is mainly determined by wanting ${ }^{46,47}$, implying that the motivational aspect of the rewarding value of food influences food intake to a higher degree than the hedonic value.

\section{Food reward, liking and wanting in the brain}

Most of the work on the actual representation of the concepts of liking and wanting in the brain has been conducted in rodents. Behaviorally, liking and wanting have been described as related processes, containing convergent and divergent parts ${ }^{30}$. The overlap as well as distinctiveness of liking and wanting have been described: processes related to wanting were found in the ventral tegmental area (VTA), nucleus of the solitary tract, ventral pallidum, ventral striatum (including the nucleus accumbens) and possibly dorsal striatum (caudate, putamen) 30,48,49,50,51,52,53. Other studies described similar regions, namely the VTA, ventral pallidum, striatum and nucleus accumbens as overlapping regions for liking and wanting, while the orbitofrontal cortex, insular cortex and amygdala were reported as more specifically liking related areas $33,46,54,55,56$. Thereby, distinctiveness and partial overlap of liking versus wanting in the central nervous system seems to involve regions similar to the homeostatic versus non-homeostatic pathways described earlier: Wanting, although influenced by some homeostatic signals, is predominantly related to the nonhomeostatic motivational pathways, and may therefore be involved specifically in non-homeostatic eating ${ }^{30,57}$. Liking, which represents the hedonic value of food, is signaled through taste and pleasure pathways, which have been described as mostly the homeostatic pathways ${ }^{30,57}$. The integration of liking and wanting into the rewarding value may take place in higher centers, such as the orbitofrontal and 
prefrontal cortices, in which cognitive control is exerted, and which may then modulate the basal nuclei, i.e. NTS, VTA, striatal regions and NAc in a top down manner ${ }^{31}$. For successful reward related learning, it is necessary to evaluate reward outcomes, i.e. compare reward prediction with reward outcome. This comparison has been attributed to the anterior cingulate cortex, but also the nucleus accumbens, caudate and putamen $48,58,59,60,61$. Here, higher activation was found in cases were reward prediction did not match reward outcome, indicating that the error is signaled rather than the success of prediction.

To investigate food reward specifically, several types of cues were used, including food images, positive and negative affective images, smells and tastes. Using any of these stimuli, consistently the regions associated with homeostatic and nonhomeostatic signaling were activated, indicating that assessment of food choice, and liking and wanting using any of these stimuli should be feasible ${ }^{54,62,63,64,65,66}$. So far, the location of liking and wanting in the human brain has not been assessed using directly comparable paradigms. Thus while the behavioral aspects of liking and wanting have been studied in humans, the representations of these processes in the human brain have been inferred from animal research $30,67,68,69$. To assess and compare behavioral and centrally signaled liking and wanting, a set of stimuli that was comparable for liking and wanting was used. This paradigm was discriminatory between the two concepts, and the task related signaling in the brain was recorded simultaneously using functional neuroimaging.

\section{Reward models}

The relation between reward and food intake has been described with two distinct, but seemingly contradictory models. The reward sensitivity theory states that high reward, which is perceived as pleasurable, may lead to increased reward seeking. Arguably the strength of this theory is that pleasure is a necessary motivator for behavior: the more pleasurable it is to perform a given action (such as eating), the higher is the motivation to start and subsequent positive feedback supports continuation. Support for this theory has been found in human eating behavior ${ }^{70,71,72,73}$. Contrarily, the model of the reward deficiency syndrome explains reward seeking as the maintenance of a reward balance. Rather than acting on short term pleasure alone, it is thought that each individual needs to obtain a given total level of reward. Thereby subjects with lower reward sensitivity need to sustain their reward balance by increasing the frequency or intensity of the rewarding activity, such as eating ${ }^{74,75}$. With respect to obesity, lower dopamine receptor density has been observed in obese individuals as a function of $\mathrm{BMI}^{76}$. Furthermore, previous studies have shown that dopamine release in these brain areas is dependent on satiety and body mass index ${ }^{56,76}$, and recent studies showed that small genetic variations in the gene for dopamine receptors is predictive of the level of brain signaling in the reward system and body weight in the present and future ${ }^{66,77}$. Additionally, it has been shown that repeated consumption of rewarding substances (drugs, food) leads to decreased dopaminergic reward signaling ${ }^{75,78}$ and finally, several dopamine antagonists that are used to treat psychotic disorders reportedly lead to increased body weight in many individuals, by increasing energyintake $^{79}$. Taken together, low dopaminergic signaling may result in more frequent 
meals and more diverse, or more palatable food, suggesting that the dopamine reward system is directly involved in the etiology of obesity. Recently, an attempt has been made to combine both reward models, describing reward sensitivity as a function of BMI as an inverted u-shaped relation. In this model, lower body-weight is associated with reward sensitivity, while higher body weight, is associated with reward deficiency ${ }^{80}$. It is argued that individuals with high reward sensitivity initially tend to have slightly higher body weight and are at high risk for getting heavier. At a certain weight, reward deficiency develops, leading to further increased body weight due to compensatory reward seeking. This new unifying theory of reward sensitivity may offer an interesting explanation for the discrepancy for the theories on reward sensitivity, while both theories seem to be supported by some studies. Given that reward deficiency has been described in overweight and obese individuals, it is likely that achievement of reward balance in those individuals can disturb energy balance, while successful energy balance may lead to loss of reward balance. Thus these subjects cannot achieve both balances at once, leaving them prone to future weight gain.

The effects of body mass, hunger and satiety on activation of the reward system has been studied. However, it is of great interest how the processes of liking and wanting related brain signaling may relate to the behavioral liking and wanting measures, and how they are modulated by hunger, satiety and body mass.

\section{The effect of the hypothalamus pituitary (HPA) axis on the rewarding value of food}

Psychological stress has been implicated in the development of overweight and obesity, since stress indices haver risen in developed societies coinciding with increasing numbers of overweight and obese individuals ${ }^{81,82}$. This stress is indicated by increased activity of the hypothalamus pituitary adrenal axis (HPA-axis), which ultimately leads to elevated cortisol levels ${ }^{83,84}$ and it has been shown that viscerally obese subjects often have increased cortisol levels compared with normal weight subjects ${ }^{85}$. Moreover, stress appeared to result in food choice for items with a higher content of fat and sweet, which are perceived as highly rewarding 86,87,88,89,90. It was shown that stress leads to eating in the absence of hunger in subjects with high disinhibition scores ${ }^{91}$. This suggests that stress interferes with non-homeostatic pathways, such as food reward signaling, explaining a shift of food choice towards foods with high fat and carbohydrate content ${ }^{91}$. In the brain, interactions between dopamine reward systems and cortisol, and corticotropin releasing factor have been shown $92,93,94,95,96$, confirming that a state of endocrinological stress may lead to dramatic changes in food reward signaling. Overall, these findings suggest that stress related increase of cortisol may support the development of overweight and obesity.

Related to the effects of stress and HPA-axis activation, other hormones were implicated in regulating food intake, such as insulin and leptin. The obesogenic effects of leptin deficiency in rats can be reversed by subsequent adrenalectomy ${ }^{97}$, indicating that both leptin and cortisol signaling simultaneously, or co-dependently contribute to food intake regulation. Furthermore, insulin levels are positively related to cortisol levels and it has been shown that insulin and cortisol antagonistically 
affect each other in relation to feeding behavior and body weight ${ }^{98,99,100}$. More specifically, it was shown that insulin and cortisol co-determined the macronutrient intake in rats, such that higher insulin levels were associated with higher lard intake in rats ${ }^{101}$ and other studies suggest that also high cortisol levels alone lead to altered food choice $89,102,103$. While the involvement of cortisol, insulin and leptin in food intake and reward signaling has been described, it remained unclear how these hormones may affect liking and wanting behaviorally, in the brain, or the link between brain and behavior.

\section{The effects of shifts in macronutrient composition}

In studies of energy intake related to macronutrient intake, important differences between the satiating efficacies of each respective macronutrient was found. Consistently, protein was shown to have the highest satiating capacity, followed by carbohydrates and fat ${ }^{104}$, and satiety could be induced acutely with a single high protein meal, and sustained for longer periods of time when high protein meals were consumed ${ }^{104,105,106}$. Unfortunately, compliance to protein diets tends to be relatively low ${ }^{105}$. Additionally, it has been shown that extreme single macronutrient intakes during one meal, lead to macronutrient compensation, such that the overall macronutrient composition averaged across the day was close to normal over the whole day $(39-46 \%$ of Energy carbohydrates, $37-44 \%$ of Energy fat and $17 \%$ of Energy protein ${ }^{107}$ ).

Macronutrient composition of a consumed meal translates to changes in hormone levels, such as cortisol, cholecystokinine (CCK), glucagon like peptide 1 (GLP), ghrelin, insulin, leptin and peptide YY (PYY) 101,104,106,108,109,110,111,112. In the brain, each hormone has been shown to modulate food choice and energy intake. The relationship between hormone levels and hunger ratings, or energy intake are less than perfect: the predictive value of hormones for hunger, satiety, or energy intake is generally no higher than approximately $30 \% 113,114,115,116,117,118$. However, a more recent study indicated that taking timing and time shifting into account may increase the predictive power to $40-70 \%{ }^{119}$. Nonetheless, the usefulness of hormones as satiation and satiety markers is currently somewhat limited. Here, the focus was directed at the effects of different macronutrient intakes and macronutrient shifts on liking and wanting related brain activation.

\section{Functional neuroimaging}

Previously, most of our knowledge on the rewarding value of food has been obtained through animal research, often using highly invasive methods, involving brain surgery ${ }^{30}$. Until now, studies on the reward system have not specifically targeted the concepts of liking and wanting in humans using non-invasive neuroscience techniques $120,121,122,123,124$. Several methods of brain imaging and neuronal recording are available, each with its own merits and drawbacks, starting with the availability of each technique. One commonly used technique is functional magnetic resonance imaging (fMRI). This technique allows a relatively low risk assessment of neuronal activity, with a spatial resolution on the millimeter scale using the current technology (3 Tesla imaging systems ${ }^{125}$ ). Potentially, other methods offer interesting and 
exciting insights as well. For example, one alternative technique, positron emission tomography (PET) using a radioactive tracer, allows to visualize neurotransmitter receptor availability on a millimeter scale and thereby provide insight in the mechanisms of neuronal signaling ${ }^{125}$. Furthermore, electro-encephalography (EEG) or magneto-encephalography (MEG) record the electric activity of neurons (respectively directly, or by measuring the resulting magnetic field), allowing to distinguish processes that are spaced in millisecond resolution, although spatial resolution is limited and the ability to discriminate the depth of the origin of the signal is extremely limited ${ }^{125}$. The extremely high temporal resolution is in stark contrast with fMRI, which is limited in its temporal resolution to seconds using current systems and whole brain coverage ${ }^{125}$.

Using fMRI implied that a high temporal resolution and the ability to measure the underlying neuronal mechanism were sacrificed ${ }^{125}$. However, given our specific research questions on the locations of brain signaling related to liking and wanting, fMRI was chosen for its ability to visualize the subjects brain in high resolution on the single millimeter scale, thus helping to pinpoint the exact locations that are involved in specific processes, such as liking and wanting.

\section{Functional magnetic resonance imaging}

MRI imaging uses a strong magnetic field and radio-frequencies to induce a signal that is dependent on the local presence of hydrogen nuclei ${ }^{125}$. The current functional MRI method of choice, is blood oxygen level dependent (BOLD) imaging. This type of functional imaging benefits from the close relation of neuronal activity and the regulation of its regional blood supply, and the magnetic properties of de-oxygenated hemoglobin versus the non-magnetic properties of oxygenated hemoglobin ${ }^{126}$. As oxygen demand in an active brain region increases with neuronal activity, this demand is met with a higher than proportional increase in blood flow. Thereby, the capillary bed of a localized region, temporarily contains a greater proportion of oxygenized blood and thus more oxygenized hemoglobin. Consequently, the local homogeneity of the magnetic field increases slightly. By choosing a scanner setting that is specifically sensitive to magnetic field inhomogeneity (i.e. $2^{*}$ weighted imaging), a signal containing data on localized variations in blood supply is obtained. Together with the relation between neuronal activity and blood supply, neuronal activity can be inferred from the BOLD signal'126,127,128,129,130.

While in the scanner, subjects are instructed to participate in a task, such as rating food for liking and wanting, respectively. The subjects behavioral responses, as well as detailed BOLD responses are recorded continuously during the scan, and from these data the magnitude of the task related signal is assessed: The behavioral data is convolved with the known average shape of the hemodynamic response to neuronal activity, and used as a predictor in subsequent analyses ${ }^{131}$. To prepare BOLD imaging data for further analysis, two procedures are executed: Firstly, on a practical level, even when fixated, subjects cannot lie completely still, thus corrections for movement have to be applied to align all data to a fixed volumeimage (3D motion correction ${ }^{131}$ ). Secondly, the brains of different individuals need to be spatially normalized, such that the corresponding regions of all subjects are aligned with each other. This is achieved by transforming all data to a standard size- 
template, i.e. scaling each brain to match the Talairach brain ${ }^{131,132}$. Finally, the BOLD signal contains a vast amount of noise, including the effects of breathing, heart beat, movement and background brain activity that have to be accounted for as much as possible, especially since the signal associated with the intentional stimulus is a small percentage of the total measured signal. Additionally, a long stay in the fMRI scanner results in habituation effects and fatigue, resulting in progressively lower signal returns throughout the scan. Each of these multiple confounding effects, as well as the intended brain activation signal are modeled as a predictor (also often called 'regressor') in a 'general linear model' (GLM) approach ${ }^{131}$. To assist in the preparation of dummy coding, filtering of motion parameter estimates and habituation effects, a publicly available software tool was privately developed (BVAPredictor tool ${ }^{133}$ ). Finally, from the GLM, parameter estimates for each predictor or confound (i.e. beta values) are extracted for further analysis. In the final step, these estimates are then used, i.e. compared or contrasted with each other, or related to other measures, such as questionnaire results, hormone data, or subject characteristics.

\section{Functional MRI stimuli}

During the functional MRI run, subjects participate in one or more tasks. Dependent on the questions that are addressed, there are two general types of stimuli that can be used: In a blocked design, stimuli are grouped together and presented in relatively long stretches one after another. While the continuous nature of this design results in strong brain activation in relevant regions, the discriminatory capacity of this technique is limited: the resulting BOLD signal is effectively averaged over multiple stimuli ${ }^{125}$. In contrast, in an event related design, the stimuli are spaced apart by a long inter trial interval, or by shorter intervals of variable length. Thereby, the activation resulting from individual stimuli can be assessed. While an event related design can achieve much more detail regarding each individual stimulus, the overall sensitivity for small effects is lower: each stimulus presentation represents a small individual effect, that may become indiscernible due to the limited signal to noise ratio. To counter these limitations, individual stimuli must be presented a number of times, providing an average signal over multiple trials ${ }^{125}$. In the research presented in this thesis, it was chosen to use a fast event related design. This enabled subjects to change their valuation of any food item at any time during the scan. In previous studies the generally accepted approach has been to analyze and describe the brain activation that is associated with a given task; however, in most cases, the behavioral observations were not made, or not combined with the imaging data. In the present studies brain signaling was directly related to behavioral outcomes, including the liking and wanting ratings given during the task, as well as mood scores, hunger and satiety ratings, and other behavioral scores on the TFEQ. 


\section{Outline of this thesis}

Against the background of the global obesity epidemic, it is important to understand how overeating and the likely increase in body weight may develop. Therefore the focus was on the processes that may lead to loss of homeostatic regulation of food intake. Energy balance is achieved by tuning energy intake to meet energy expenditure. Energy intake, thus food intake, is affected by reward processes such as liking and wanting, as well as cognitive processes such as dietary restraint. Therefore, it was studied which brain regions may be involved in homeostatic and non-homeostatic food intake, related to liking and wanting task related signaling, to BMI, and dietary restraint (Chapter 2). In the brain, a distinct representation of liking and wanting was expected, in which wanting was to be represented in regions related to reward and energy homeostasis (e.g. in the striatum including nucleus accumbens and hypothalamus), while liking was expected in regions related to taste, reward, and affectivity (e.g. in the insular cortex, nucleus accumbens, and amygdala). Furthermore, cognitive dietary restraint may play a key role in behavioral dietary restriction, therefore it was hypothesized that dietary restraint may modulate the rewarding value of food depending on the energy state. Previous reports seemed contradictory regarding the way in which the rewarding value of food may be related to body weight as a cause or effect. Therefore, the aim was to determine a model that would consistently link the rewarding value of food (i.e. liking and wanting) to a range of body weights. This was studied in normal weight subjects (Chapter 2) as well as in subjects with a wider range of body weight (BMI of 19-33), while linear and nonlinear approaches were used to determine the best model fit (Chapter 3).

Stress and the associated increase in HPA-axis activity has been implicated as a cause for eating in the absence of hunger, which is non-homeostatic eating. Additional evidence pointed toward changes in reward related brain signaling as an effect of higher levels of cortisol and $\mathrm{CRH}$. Therefore the effects of acute psychosocial stress on food choice related brain activity was studied. It was hypothesized that acute stress reduces signaling in food reward circuits, which would then lead to non-homeostatic eating and possibly altered food choice (Chapter 4). In addition, it was studied how high versus low HPA-axis activation affects the link between behaviorally liking and wanting (i.e. explicitly expressed), and liking and wanting task related signaling. The hypothesis was that behavioral liking and wanting is represented in relevant brain areas during low HPA-axis activation, while during high HPA-activation this link may be disturbed. This would help to explain altered food preference and food intake that has been described during stress (Chapter 5).

Finally, it has been shown, that extreme macronutrient intakes may lead to compensatory food choice. Therefore, the effects of acute high protein and high carbohydrate intakes, with subsequent shifts in macronutrient compositions were studied and related to liking and wanting related brain signaling. Given the satiating properties of protein, thus involvement of homeostatic regulation in addition to possible reward related influences, it was hypothesized that differential shifts in protein versus carbohydrate intake, may acutely modulate hypothalamic and striatal signaling, which may represent differential signaling of motivation for energy intake in the absence of hunger (Chapter 6). 


\section{References}

1. Catenacci, V.A., Hill, J.O. \& Wyatt, H.R. The Obesity Epidemic. Clinics in Chest Medicine 30, 415-444 (2009).

2. James, P.T. Obesity: The Worldwide Epidemic. Clin Dermatol 22, 276-280 (2004).

3. James, P.T., Leach, R., Kalamara, E. \& Shayeghi, M. The Worldwide Obesity Epidemic. Obes Res 9 Suppl 4, 228S-233S (2001).

4. Despres, J.P. \& Lemieux, I. Abdominal Obesity and Metabolic Syndrome. Nature 444, 881-887 (2006).

5. Hutley, L. \& Prins, J.B. Fat as an Endocrine Organ: Relationship to the Metabolic Syndrome. Am J Med Sci 330, 280-289 (2005).

6. James, W.P.T. Who Recognition of the Global Obesity Epidemic. Int J Obes 32, S120-S126 (2008).

7. Berthoud, H.R. Neural Control of Appetite: Cross-Talk between Homeostatic and Non-Homeostatic Systems. Appetite 43, 315-317 (2004).

8. Selassie, M. \& Sinha, A.C. The Epidemiology and Aetiology of Obesity: A Global Challenge. Best Practice \& Research Clinical Anaesthesiology 25, 1-9 (2011).

9. Giskes, K., van Lenthe, F., Avendano-Pabon, M. \& Brug, J. A Systematic Review of Environmental Factors and Obesogenic Dietary Intakes among Adults: Are We Getting Closer to Understanding Obesogenic Environments? Obesity Reviews 12, e95-e106 (2011).

10. Westerterp, K.R. \& Speakman, J.R. Physical Activity Energy Expenditure Has Not Declined since the 1980s and Matches Energy Expenditures of Wild Mammals. Int J Obes 32, 1256-1263 (2008).

11. Steenhuis, I.H., Leeuwis, F.H. \& Vermeer, W.M. Small, Medium, Large or Supersize: Trends in Food Portion Sizes in the Netherlands. Public Health Nutrition 13, 852-857 (2010).

12. Piernas, C. \& Popkin, B.M. Food Portion Patterns and Trends among U.S. Children and the Relationship to Total Eating Occasion Size, 1977-2006. J Nutr 141, 1159-1164 (2011).

13. Berthoud, H.R. Mind Versus Metabolism in the Control of Food Intake and Energy Balance. Physiol Behav 81, 781-793 (2004).

14. Edholm, O.G. Energy Balance in Man Studies Carried out by the Division of Human Physiology, National Institute for Medical Research. J Hum Nutr 31, 413-431 (1977).

15. Berthoud, H.-R. Multiple Neural Systems Controlling Food Intake and Body Weight. Neuroscience \& Biobehavioral Reviews 26, 393-428 (2002).

16. Erlanson-Albertsson, C. How Palatable Food Disrupts Appetite Regulation. Basic Clin Pharmacol Toxicol 97, 61-73 (2005).

17. Berthoud, H.R. Neural Systems Controlling Food Intake and Energy Balance in the Modern World. Curr Opin Clin Nutr Metab Care 6, 615-620 (2003).

18. Langhans, W. \& Geary, N. Overview of the Physiological Control of Eating. Forum Nutr 63, 9-53 (2010).

19. Moran, T.H. \& Dailey, M.J. Intestinal Feedback Signaling and Satiety. Physiology \& Behavior In Press (2011).

20. Schwartz, G.J. Brainstem Integrative Function in the Central Nervous System Control of Food Intake. Forum Nutr 63, 141-151 (2010)

21. Zheng, H., Lenard, N.R., Shin, A.C. \& Berthoud, H.R. Appetite Control and Energy Balance Regulation in the Modern World: Reward-Driven Brain Overrides Repletion Signals. Int J Obes (Lond) 33 Suppl 2, S8-13 (2009).

22. Blevins, J.E. \& Baskin, D.G. Hypothalamic-Brainstem Circuits Controlling Eating. Forum Nutr 63, 133-140 (2010).

23. Banks, W.A. Blood-Brain Barrier as a Regulatory Interface. Forum Nutr 63, 102-110 (2010).

24. Moran, T.H. Hypothalamic Nutrient Sensing and Energy Balance. Forum Nutr 63, 94-101 (2010).

25. Munzberg, H. Leptin-Signaling Pathways and Leptin Resistance. Forum Nutr 63, 123-132 (2010).

26. Williams, G., Harrold, J.A. \& Cutler, D.J. The Hypothalamus and the Regulation of Energy Homeostasis: Lifting the Lid on a Black Box. Proceedings of the Nutrition Society 59, 385-396 (2000).

27. Hoebel, B.G. Feeding: Neural Control of Intake. Annual Review of Physiology 33, 533-568 (1971).

28. Wise, R.A. Lateral Hypothalamic Electrical Stimulation: Does It Make Animals 'Hungry'? Brain Research 67, 187-209 (1974).

29. Nargund, R.P., Strack, A.M. \& Fong, T.M. Melanocortin-4 Receptor (Mc4r) Agonists for the Treatment of Obesity. Journal of Medicinal Chemistry 49, 4035-4043 (2006).

30. Berridge, K.C. Food Reward: Brain Substrates of Wanting and Liking. Neurosci Biobehav Rev 20, 1-25 (1996).

31. Berthoud, H.R. Homeostatic and Non-Homeostatic Pathways Involved in the Control of Food Intake and Energy Balance. Obesity (Silver Spring) 14 Suppl 5, 197S-200S (2006).

32. Balleine, B.W., Delgado, M.R. \& Hikosaka, O. The Role of the Dorsal Striatum in Reward and Decision-Making. J. Neurosci. 27, 8161-8165 (2007).

33. Kelley, A.E., Baldo, B.A., Pratt, W.E. \& Will, M.J. Corticostriatal-Hypothalamic Circuitry and Food Motivation: Integration of Energy, Action and Reward. Physiology \& Behavior 86, 773-795 (2005).

34. Sánchez-Lasheras, C., Christine Könner, A. \& Brüning, J.C. Integrative Neurobiology of Energy HomeostasisNeurocircuits, Signals and Mediators. Frontiers in Neuroendocrinology 31, 4-15 (2010).

35. Ahlskog, J.E. \& Hoebel, B.G. Overeating and Obesity from Damage to a Noradrenergic System in the Brain. Science 182, 166-169 (1973).

36. Brook, C. \& Iversen, S.D. Changed Eating and Locomotor Behaviour in the Rat after 6-Hydroxydopamine Lesions to the Substantia Nigra. Neuropharmacology 14, 95-105 (1975). 
37. Wise, R., Spindler, J., deWit, H. \& Gerberg, G. Neuroleptic-Induced "Anhedonia" In Rats: Pimozide Blocks Reward Quality of Food. Science 201, 262-264 (1978).

38. Strassnig, M., Miewald, J., Keshavan, M. \& Ganguli, R. Weight Gain in Newly Diagnosed First-Episode Psychosis Patients and Healthy Comparisons: One-Year Analysis. Schizophrenia Research 93, 90-98 (2007).

39. Bechara, A. \& Van Der Linden, M. Decision-Making and Impulse Control after Frontal Lobe Injuries. Current opinion in neurology $18,734-739$ (2005).

40. Nieuwenhuis, I.L.C. \& Takashima, A. The Role of the Ventromedial Prefrontal Cortex in Memory Consolidation. Behavioural Brain Research 218, 325-334 (2011).

41. Stunkard, A.J. \& Messick, S. The Three-Factor Eating Questionnaire to Measure Dietary Restraint, Disinhibition and Hunger. J Psychosom Res 29, 71-83 (1985).

42. Pasman, W.J., Saris, W.H. \& Westerterp-Plantenga, M.S. Predictors of Weight Maintenance. Obes Res 7, 43-50 (1999).

43. Westerterp-Plantenga, M.S., Kempen, K.P. \& Saris, W.H. Determinants of Weight Maintenance in Women after Diet-Induced Weight Reduction. Int J Obes Relat Metab Disord 22, 1-6 (1998).

44. Rutters, F., Nieuwenhuizen, A.G., Lemmens, S.G.T., Born, J.M. \& Westerterp-Plantenga, M.S. Acute StressRelated Changes in Eating in the Absence of Hunger. Obesity 17, 72-72 (2009).

45. Robinson, T.E. \& Berridge, K.C. Addiction. Annu Rev Psychol 54, 25-53 (2003).

46. Epstein, L.H., Leddy, J.J., Temple, J.L. \& Faith, M.S. Food Reinforcement and Eating: A Multilevel Analysis. Psychological bulletin 133, 884-906 (2007).

47. Born, J.M., Lemmens, S.G.T., Formisano, E., Goebel, R. \& Westerterp-Plantenga, M.S. Differences between Liking and Wanting Signals in the Human Brain and Relations with Bmi and Cognitive Dietary Restraint. Am J Clin Nutr (2011)

48. Abler, B., Walter, H., Erk, S., Kammerer, H. \& Spitzer, M. Prediction Error as a Linear Function of Reward Probability Is Coded in Human Nucleus Accumbens. Neuroimage 31, 790 - 795 (2006).

49. Born, J.M., et al. Acute Stress and Food-Related Reward Activation in the Brain During Food Choice During Eating in the Absence of Hunger. Int J Obes 34, 172-181 (2010).

50. Bratcher, N.A., Farmer-Dougan, V., Dougan, J.D., Heidenreich, B.A. \& Garris, P.A. The Role of Dopamine in Reinforcement: Changes in Reinforcement Sensitivity Induced by D1-Type, D2-Type, and Nonselective Dopamine Receptor Agonists. J Exp Anal Behav 84, 371-399 (2005).

51. McCabe, C., Cowen, P. \& Harmer, C. Neural Representation of Reward in Recovered Depressed Patients. Psychopharmacology 205, 667-677 (2009).

52. Murray, E.A. The Amygdala, Reward and Emotion. Trends in cognitive sciences 11, $489-497$ (2007).

53. Evans, A.H., et al. Compulsive Drug Use Linked to Sensitized Ventral Striatal Dopamine Transmission. Ann Neurol 59, 852-858 (2006).

54. Gottfried, J.A., O'Doherty, J. \& Dolan, R.J. Encoding Predictive Reward Value in Human Amygdala and Orbitofrontal Cortex. Science 301, 1104-1107 (2003).

55. Kelley, A.E., et al. Opioid Modulation of Taste Hedonics within the Ventral Striatum. Physiology \& Behavior 76, 365 (2002).

56. Small, D.M., Jones-Gotman, M. \& Dagher, A. Feeding-Induced Dopamine Release in Dorsal Striatum Correlates with Meal Pleasantness Ratings in Healthy Human Volunteers. Neuroimage 19, 1709-1715 (2003).

57. Berridge, K.C. 'Liking' and 'Wanting' Food Rewards: Brain Substrates and Roles in Eating Disorders. Physiology \& Behavior 97, 537-550 (2009).

58. Haruno, M. \& Kawato, M. Different Neural Correlates of Reward Expectation and Reward Expectation Error in the Putamen and Caudate Nucleus During Stimulus-Action-Reward Association Learning. J Neurophysiol 95 948-959 (2006).

59. Holroyd, C.B. \& Coles, M.G.H. Dorsal Anterior Cingulate Cortex Integrates Reinforcement History to Guide Voluntary Behavior. Cortex; a journal devoted to the study of the nervous system and behavior 44, 548-559 (2008).

60. Knutson, B. \& Cooper, J.C. Functional Magnetic Resonance Imaging of Reward Prediction. Current opinion in neurology 18, 411-417 (2005).

61. Preuschoff, K., Quart, S. \& Bossaerts, P. Human Insula Activation Reflects Risk Prediction Errors as Well as Risk. J Neurosci 28, 2745 - 2752 (2008).

62. Del Parigi, A., et al. Neuroimaging and Obesity: Mapping the Brain Responses to Hunger and Satiation in Humans Using Positron Emission Tomography. Annals of the New York Academy of Sciences 967, 389-397 (2002).

63. Gautier, J.F., et al. Effect of Satiation on Brain Activity in Obese and Lean Women. Obes Res 9, $676-684$ (2001).

64. O'Doherty, J.P. Reward Representations and Reward-Related Learning in the Human Brain: Insights from Neuroimaging. Curr Opin Neurobiol 14, 769-776 (2004).

65. Matsuda, M., et al. Altered Hypothalamic Function in Response to Glucose Ingestion in Obese Humans. Diabetes 48, 1801-1806 (1999).

66. Stice, E., Spoor, S., Bohon, C. \& Small, D.M. Relation between Obesity and Blunted Striatal Response to Food Is Moderated by Taqia A1 Allele. Science 322, 449-452 (2008).

67. Lemmens, S.G.T., et al. Eating What You Like Induces a Stronger Decrease of 'Wanting' to Eat. Physiology \& Behavior 98, 318-325 (2009). 
68. Lemmens, S.G., Rutters, F., Born, J.M. \& Westerterp-Plantenga, M.S. Stress Augments Food 'Wanting' and Energy Intake in Visceral Overweight Subjects in the Absence of Hunger. Physiology \& Behavior 103, $157-163$ (2010).

69. Lemmens, S.G., et al. Dietary Restraint and Control over 'Wanting'following Consumption of 'Forbidden' Food. Obesity 18, 1926-1931 (2010).

70. Schienle, A., Schäfer, A., Hermann, A. \& Vaitl, D. Binge-Eating Disorder: Reward Sensitivity and Brain Activation to Images of Food. Biological Psychiatry 65, 654-661 (2008).

71. Martin, L.E. \& Potts, G.F. Reward Sensitivity in Impulsivity. Neuroreport 15, 1519-1522 (2004).

72. Davis, C., et al. From Motivation to Behaviour: A Model of Reward Sensitivity, Overeating, and Food Preferences in the Risk Profile for Obesity. Appetite 48, 12-19 (2007).

73. Volkow, N.D., et al. "Nonhedonic" Food Motivation in Humans Involves Dopamine in the Dorsal Striatum and Methylphenidate Amplifies This Effect. Synapse 44, 175-180 (2002).

74. Blum, K., et al. Reward Deficiency Syndrome: A Biogenetic Model for the Diagnosis and Treatment of Impulsive, Addictive, and Compulsive Behaviors. J Psychoactive Drugs 32 Suppl, i-iv, 1-112 (2000).

75. Kenny, P.J. Brain Reward Systems and Compulsive Drug Use. Trends Pharmacol Sci 28, 135-141 (2007).

76. Wang, G.J., et al. Brain Dopamine and Obesity. Lancet 357, 354-357 (2001).

77. Stice, E., Yokum, S., Bohon, C., Marti, N. \& Smolen, A. Reward Circuitry Responsivity to Food Predicts Future Increases in Body Mass: Moderating Effects of Drd2 and Drd4. Neuroimage 50, 1618-1625 (2010).

78. Koob, G.F. \& Volkow, N.D. Neurocircuitry of Addiction. Neuropsychopharmacology 35, 217-238 (2009).

79. Allison, D.B., et al. Antipsychotic-Induced Weight Gain: A Comprehensive Research Synthesis. Am J Psychiatry 156, 1686-1696 (1999).

80. Davis, C. \& Fox, J. Sensitivity to Reward and Body Mass Index (Bmi): Evidence for a Non-Linear Relationship. Appetite 50, 43-49 (2008).

81. Epel, E.S., et al. Stress and Body Shape: Stress-Induced Cortisol Secretion Is Consistently Greater among Women with Central Fat. Psychosomatic medicine 62, 623-632 (2000).

82. Kyrou, I., Chrousos, G.P. \& Tsigos, C. Stress, Visceral Obesity, and Metabolic Complications. Annals of the New York Academy of Sciences 1083, 77-110 (2006).

83. Axelrod, J. \& Reisine, T.D. Stress Hormones: Their Interaction and Regulation. Science 224, 452-459 (1984).

84. Eechaute, W., Lacroix, E. \& Leusen, I. [Correlation between the Plasma Level of Free Corticosterone and in Vitro Adrenal Activity in the Rat.]. Archives internationales de physiologie et de biochimie 71, 528-533 (1963).

85. Bjorntorp, P. \& Rosmond, R. Obesity and Cortisol. Nutrition 16, 924-936 (2000).

86. Drewnowski, A., Krahn, D.D., Demitrack, M.A., Nairn, K. \& Gosnell, B.A. Taste Responses and Preferences for Sweet High-Fat Foods: Evidence for Opioid Involvement. Physiol Behav 51, 371-379 (1992).

87. Elfhag, K. \& Erlanson-Albertsson, C. Sweet and Fat Taste Preference in Obesity Have Different Associations with Personality and Eating Behavior. Physiol Behav 88, 61-66 (2006).

88. Epel, E., Lapidus, R., McEwen, B. \& Brownell, K. Stress May Add Bite to Appetite in Women: A Laboratory Study of Stress-Induced Cortisol and Eating Behavior. Psychoneuroendocrinology 26, 37-49 (2001).

89. Oliver, G., Wardle, J. \& Gibson, E.L. Stress and Food Choice: A Laboratory Study. Psychosomatic medicine 62, 853-865 (2000).

90. Salbe, A.D., DelParigi, A., Pratley, R.E., Drewnowski, A. \& Tataranni, P.A. Taste Preferences and Body Weight Changes in an Obesity-Prone Population. Am J Clin Nutr 79, 372-378 (2004).

91. Rutters, F., Nieuwenhuizen, A.G., Lemmens, S.G.T., Born, J.M. \& Westerterp-Plantenga, M.S. Acute StressRelated Changes in Eating in the Absence of Hunger. Obesity (2008).

92. Fulton, S., Richard, D., Woodside, B. \& Shizgal, P. Interaction of Crh and Energy Balance in the Modulation of Brain Stimulation Reward. Behav Neurosci 116, 651-659 (2002).

93. Pani, L., Porcella, A. \& Gessa, G.L. The Role of Stress in the Pathophysiology of the Dopaminergic System. Mol Psychiatry 5, 14-21 (2000).

94. Pruessner, J.C., Champagne, F., Meaney, M.J. \& Dagher, A. Dopamine Release in Response to a Psychological Stress in Humans and Its Relationship to Early Life Matemal Care: A Positron Emission Tomography Study Using [11c]Raclopride. J Neurosci 24, 2825-2831 (2004).

95. Salamone, J.D., Cousins, M.S. \& Snyder, B.J. Behavioral Functions of Nucleus Accumbens Dopamine: Empirical and Conceptual Problems with the Anhedonia Hypothesis. Neurosci Biobehav Rev 21, 341-359 (1997).

96. Czyrak, A., Mackowiak, M., Chocyk, A., Fijal, K. \& Wedzony, K. Role of Glucocorticoids in the Regulation of Dopaminergic Neurotransmission. Pol J Pharmacol 55, 667-674 (2003).

97. Makimura, H., et al. Adrenalectomy Reverses Obese Phenotype and Restores Hypothalamic Melanocortin Tone in Leptin-Deficient Ob/Ob Mice. Diabetes 49, 1917-1923 (2000).

98. Dallman, M.F., et al. Feast and Famine: Critical Role of Glucocorticoids with Insulin in Daily Energy Flow. Frontiers in Neuroendocrinology 14, 303 (1993).

99. Strack, A.M., Sebastian, R.J., Schwartz, M.W. \& Dallman, M.F. Glucocorticoids and Insulin: Reciprocal Signals for Energy Balance. Am J Physiol 268, R142-149 (1995).

100. la Fleur, S.E. The Effects of Glucocorticoids on Feeding Behavior in Rats. Physiol Behav 89, 110-114 (2006).

101. la Fleur, S.E., Akana, S.F., Manalo, S.L. \& Dallman, M.F. Interaction between Corticosterone and Insulin in Obesity: Regulation of Lard Intake and Fat Stores. Endocrinology 145, 2174-2185 (2004). 
102. Newman, E., O'Connor, D.B. \& Conner, M. Daily Hassles and Eating Behaviour: The Role of Cortisol Reactivity Status. Psychoneuroendocrinology 32, 125-132 (2007).

103. Wardle, J., Steptoe, A., Oliver, G. \& Lipsey, Z. Stress, Dietary Restraint and Food Intake. J Psychosom Res 48, 195-202 (2000).

104. Veldhorst, M., et al. Protein-Induced Satiety: Effects and Mechanisms of Different Proteins. Physiology \& Behavior 94, 300-307 (2008).

105. Soenen, S. \& Westerterp-Plantenga, M.S. Proteins and Satiety: Implications for Weight Management. Curr Opin Clin Nutr Metab Care 11, 747-751 (2008).

106. Lejeune, M.P., Westerterp, K.R., Adam, T.C., Luscombe-Marsh, N.D. \& Westerterp-Plantenga, M.S. Ghrelin and Glucagon-Like Peptide 1 Concentrations, 24-H Satiety, and Energy and Substrate Metabolism During a HighProtein Diet and Measured in a Respiration Chamber. Am J Clin Nutr 83, 89-94 (2006).

107. Westerterp-Plantenga, M.S., MJ, I.J. \& Wijckmans-Duijsens, N.E. The Role of Macronutrient Selection in Determining Patterns of Food Intake in Obese and Non-Obese Women. Eur J Clin Nutr 50, 580-591 (1996).

108. Wynne, K., Stanley, S., McGowan, B. \& Bloom, S. Appetite Control. J Endocrinol 184, 291-318 (2005).

109. Pecoraro, N., Reyes, F., Gomez, F., Bhargava, A. \& Dallman, M.F. Chronic Stress Promotes Palatable Feeding, Which Reduces Signs of Stress: Feedforward and Feedback Effects of Chronic Stress. Endocrinology 145, 3754-3762 (2004).

110. Figlewicz, D.P. \& Benoit, S.C. Insulin, Leptin, and Food Reward: Update 2008. Am J Physiol Regul Integr Comp Physiol 296, R9-19 (2009).

111. Martens, M.J.I., Rutters, F., Lemmens, S.G.T., Born, J.M. \& Westerterp-Plantenga, M.S. Effects of Single Macronutrients on Serum Cortisol Concentrations in Normal Weight Men. Physiology \& Behavior 101, 563-567 (2010)

112. Fulton, S., Woodside, B. \& Shizgal, P. Does Neuropeptide Y Contribute to the Modulation of Brain Stimulation Reward by Chronic Food Restriction? Behavioural Brain Research 134, 157-164 (2002).

113. Diepvens, K., Haberer, D. \& Westerterp-Plantenga, M. Different Proteins and Biopeptides Differently Affect Satiety and Anorexigenic/Orexigenic Hormones in Healthy Humans. Int J Obes 32, 510-518 (2007).

114. Erdmann, J., Hebeisen, Y., Lippl, F., Wagenpfeil, S. \& Schusdziarra, V. Food Intake and Plasma Ghrelin Response During Potato-, Rice- and Pasta-Rich Test Meals. European Journal of Nutrition 46, 196-203 (2007).

115. Smeets, A.J., Soenen, S., Luscombe-Marsh, N.D., Ueland, ñ. \& Westerterp-Plantenga, M.S. Energy Expenditure, Satiety, and Plasma Ghrelin, Glucagon-Like Peptide 1, and Peptide Tyrosine-Tyrosine Concentrations Following a Single High-Protein Lunch. The Journal of Nutrition 138, 698-702 (2008).

116. le Roux, C.W., et al. Attenuated Peptide Yy Release in Obese Subjects Is Associated with Reduced Satiety. Endocrinology 147, 3-8 (2006).

117. Guo, Y., et al. Physiological Evidence for the Involvement of Peptide Yy in the Regulation of Energy Homeostasis in Humans[Ast]. Obesity 14, 1562-1570 (2006).

118. Adam, T.C., Lejeune, M.P. \& Westerterp-Plantenga, M.S. Nutrient-Stimulated Glucagon-Like Peptide 1 Release after Body-Weight Loss and Weight Maintenance in Human Subjects. Br J Nutr 95, 160-167 (2006).

119. Lemmens, S.G.T., Martens, E.A.P., Kester, A.D.M. \& westerterp-Plantenga, M.S. Changes in Gut Hormone and Glucose Concentrations in Relation to Hunger and Fullness. Am J Clin Nutr In press (2011).

120. Adam, T.C. \& Epel, E.S. Stress, Eating and the Reward System. Physiology \& Behavior 91, 449-458 (2007).

121. Di Chiara, G. \& Bassareo, V. Reward System and Addiction: What Dopamine Does and Doesn't Do. Current Opinion in Pharmacology 7, 69 (2007).

122. Schultz, W. Predictive Reward Signal of Dopamine Neurons. J Neurophysiol 80, 1-27 (1998).

123. Schultz, W. Reward Signaling by Dopamine Neurons. Neuroscientist 7, 293-302 (2001).

124. Hoebel, B.G. Brain Neurotransmitters in Food and Drug Reward. Am J Clin Nutr 42, 1133-1150 (1985).

125. Huettel, S.A., Song, A.W. \& McCarthy, G. Functional Magnetic Resonance Imaging (Sinauer Associates, Inc, Sunderland, MA, 2004).

126. Ogawa, S., Lee, T.M., Kay, A.R. \& Tank, D.W. Brain Magnetic Resonance Imaging with Contrast Dependent on Blood Oxygenation. Proceedings of the National Academy of Sciences 87, 9868-9872 (1990).

127. Heeger, D., Huk, A., Geisler, W. \& Albrecht, D. Spikes Versus Bold: What Does Neuroimaging Tell Us About Neuronal Activity? Nat Neurosci 3, 631 - 633 (2000).

128. Yang, X., Hyder, F. \& Shulman, R.G. Functional MRI Bold Signal Coincides with Electrical Activity in the Rat Whisker Barrels. Magn Reson Med 38, 874-877 (1997).

129. Ogawa, S., et al. Functional Brain Mapping by Blood Oxygenation Level-Dependent Contrast Magnetic Resonance Imaging. A Comparison of Signal Characteristics with a Biophysical Model. Biophysical Journal 64, 803-812 (1993).

130. Logothetis, N.K. The Neural Basis of the Blood-Oxygen-Level-Dependent Functional Magnetic Resonance Imaging Signal. Philos Trans R Soc Lond B Biol Sci 357, 1003-1037 (2002).

131. Goebel, R., Esposito, F. \& Formisano, E. Analysis of Functional Image Analysis Contest (Fiac) Data with Brainvoyager Qx: From Single-Subject to Cortically Aligned Group General Linear Model Analysis and SelfOrganizing Group Independent Component Analysis. Human Brain Mapping 27, 392-401 (2006).

132. Talairach, J. \& Tournoux, P. Co-Planar Stereotaxic Atlas of the Human Brain (Thieme, Stuttgart, 1988).

133. Born, J.M. Brainvoyager Analysis Predictor Tool (Bva-Predictor Tool). http://support.brainvoyager.com/availabletools/53-user-developed-tools/238-brainvoyager-analysis-predictor-tool.html (2008-2011). 
Chapter 2

\section{Differences between liking and wanting signals in the human brain and relations with cognitive dietary restraint and body mass index}

Jurriaan M Born, Sofie GT Lemmens, Mieke JI Martens, Elia Formisano, Rainer Goebel, and Margriet S Westerterp-Plantenga, Am J Clin Nutr 2011; 94, 392-403 


\section{Abstract}

Background: Eating behavior is determined to a significant extent by the rewarding value of food i.e. liking and wanting.

Aim: To determine brain regions involved in liking and wanting and to relate brain signaling to BMI and dietary restraint.

Methods: Fifteen normal weight female subjects (Age $=21.5 \pm 0.4, \mathrm{BMl}=22.2 \pm 0.2$ ) completed a food choice paradigm using visually displayed food items during fMRI scans. Two scans were made: one in a fasted condition and one in a satiated condition. The paradigm discriminated between liking and wanting, and subjects were offered items rated highly for wanting immediately after each scan. Imaging contrasts for high/low liking and wanting were made and the data for regions of interest were extracted. Activation related to liking and wanting (respectively) was determined. Outcomes were correlated to cognitive dietary restraint and BMI.

Results: Dietary restraint predicted liking task related signaling (TRS) in the amygdala, striatum, thalamus and cingulate-cortex $(r=-0.5 \pm 0.03, p<0.00001)$. Surprisingly, in the nucleus accumbens, the pre-meal liking and wanting TRS and pre- to post-meal liking TRS change correlated positively with dietary restraint (bilateral average $r=0.6 \pm 0.02, p<0.04$ Bonferroni corrected). BMI and hunger predicted wanting TRS in the hypothalamus and the striatum $(p<0.05)$. Post-meal liking TRS in the striatum, anterior insula and cingulate cortex, and also wanting TRS in the striatum, predicted energy intake (liking $r=-0.3 \pm 0.05, p<0.0001$ and wanting $r=-0.3 \pm 0.03, p<0.00001$ ).

Conclusions: Successful dietary restraint was supported by liking TRS from pre- to post-meal in the nucleus accumbens. Reward-related signaling was inversely related to BMI and energy intake, indicating reward deficiency. 


\section{Introduction}

The prevalence of obesity and its co-morbidities is increasing in the developed world $^{1,2}$. This is caused by energy intake exceeding energy expenditure, i.e. positive energy balance ${ }^{3,4}$ and may be influenced by features of food intake, such as eating in the absence of hunger ${ }^{5}$. Conversely, overeating may be countered by cognitive dietary restraint (Three factor eating questionnaire, TFEQ ${ }^{6,7,8}$ ) and modulated by disinhibition (loss of restraint), and feelings of hunger. Together, these factors predict individual success in restricting energy intake: Relatively high dietary restraint (TFEQ F1), low disinhibition and low feelings of hunger TFEQ F2 and F3, respectively ${ }^{6,7}$, indicate success. Overeating is often driven by reward seeking ${ }^{9}$ and it remains unclear how successful and unsuccessful dietary restraint are balanced in relation to body weight regulation. Berridge's model divides the rewarding value of food into two distinct processes: 'liking', i.e. hedonic preference, or palatability, and 'wanting', the motivation to eat a particular food item ${ }^{10}$. The original animal work was translated to adult humans, using either implicit or explicit measures ${ }^{11,12,13,14}$ and our approach was based on the latter. Liking and wanting are related and may occur in synchrony, but they are not the same process: individuals may want a food item without liking it ${ }^{10,15}$. Energy intake is mainly determined by wanting ${ }^{9}$. In the brain, overlap and distinctiveness of liking and wanting are represented: wanting related processes were observed in the ventral pallidum and striatum (including caudate, globus pallidus, putamen, nucleus accumbens) in rodents and humans ${ }^{10,16,17,18,19,20}$. Overlapping areas for liking and wanting were observed in the ventral pallidum and parts of the striatum, while the orbitofrontal cortex, insular cortex and amygdala were reported as liking specific areas in rodents ${ }^{9,21,22,23,24}$. Two models describe the relationship between body weight and reward: The reward sensitivity theory, stating that high reward leads to seeking of more reward 25,26 and the reward deficiency syndrome ${ }^{17,27}$, explaining reward seeking as maintaining reward homeostasis: subjects with lower reward sensitivity need to increase the frequency or intensity of the rewarding activity 28,29 . Reward deficiency was shown as inverse relationship of dopamine receptor density and $\mathrm{BMI}$ in obese individuals ${ }^{27}$, or decreased dopaminergic reward signaling after repeated consumption of rewarding substances drugs, food ${ }^{29,30}$. Furthermore, dopamine antagonists lead to increased body weight by increasing energy intake in many individuals ${ }^{31}$.

We expected distinct representation of liking and wanting: wanting representation especially in reward and energy homeostasis related regions (striatum and hypothalamus), and liking representation in taste, reward and affectivity related regions (insular cortex, nucleus accumbens and amygdala). Cognitive dietary restraint was hypothesized to modulate the rewarding value of food depending on energy state to support dietary restriction. Finally, we expected an inverse relation of BMI and signaling in reward and energy homeostasis related areas (striatum and hypothalamus) in line with reward deficiency.

\section{Subjects and methods}

\section{Subjects}

The study was approved and registered under MEC07-3-019 by the Medical Ethical Committee of Maastricht University and at the Central Committee on Research 
Involving Human Subjects (CCMO) under NL17946.068.07. Starting on March 17th 2008, 20 test subjects were screened. Informed, written consent was obtained from all subjects. Inclusion criteria were: healthy female subjects, normal body weight (BMI 19-26) and right-handedness. Exclusion criteria were recent dieting, a personal or familial history of psychiatric disorder, or intra-uterine contraceptives. Fifteen subjects were included with the following characteristics: Age $=21.5 \pm 0.4, \mathrm{BMI}=$ 22.2 \pm 0.2 , range 19.5-25.5, Three factor eating questionnaire TFEQ, 8 scores were dietary restraint $(F 1)=9.7 \pm 1.4$, disinhibition $(F 2)=5.4 \pm 0.4$, emotional eating $(F 3)=$ $4.5 \pm 0.5$.

\section{Visual analog scale questionnaires hunger and satiety}

The Visual analog scale (VAS) questionnaires consisted of $100 \mathrm{~mm}$ lines, anchored with "not at all" at the far left "extremely" at the far right. Questions asked were "How

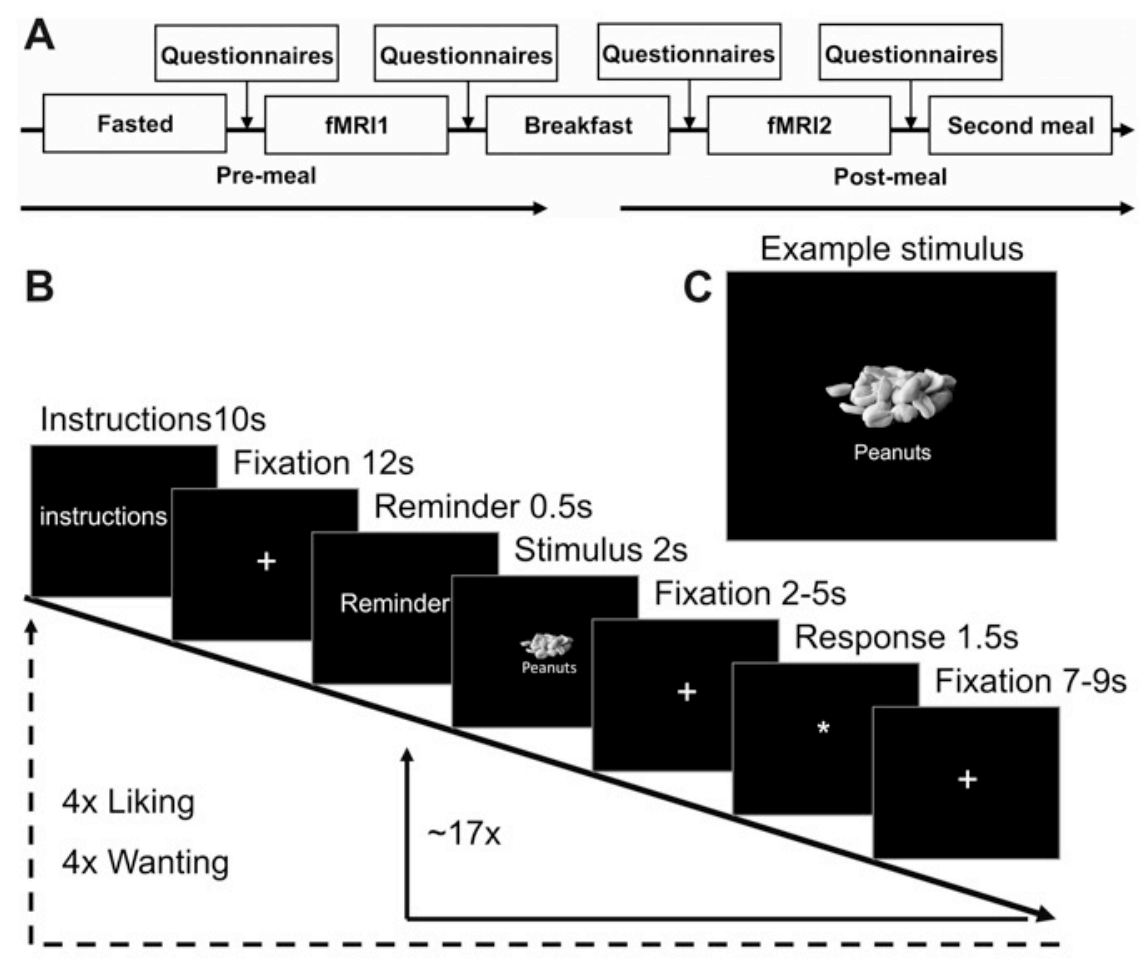

Figure 1: The test setup:

A: Schematic overview of the test day.

Subjects arrived fasted between 0800 and 0900. After $20 \mathrm{~min}$, the first questionnaires for hunger and satiety were given. Each magnetic resonance imaging (MRI) session lasted for '45 min, including a 35-min functional MRI (fMRI) run. In between scans, subjects were given 30 min to consume their meal. B: Timeline of the stimulus presentation during the fMRI run. Wanting and liking stimuli were shown in 4 segments (dotted line) that contained '17 stimulus trials in an event-related design. Solid lines indicate the course per segment. Segments were presented in a counterbalanced order. C: Example stimulus that shows the layout of the stimuli slides with a centered food image that was standardized for size and color with the food name below the image. 


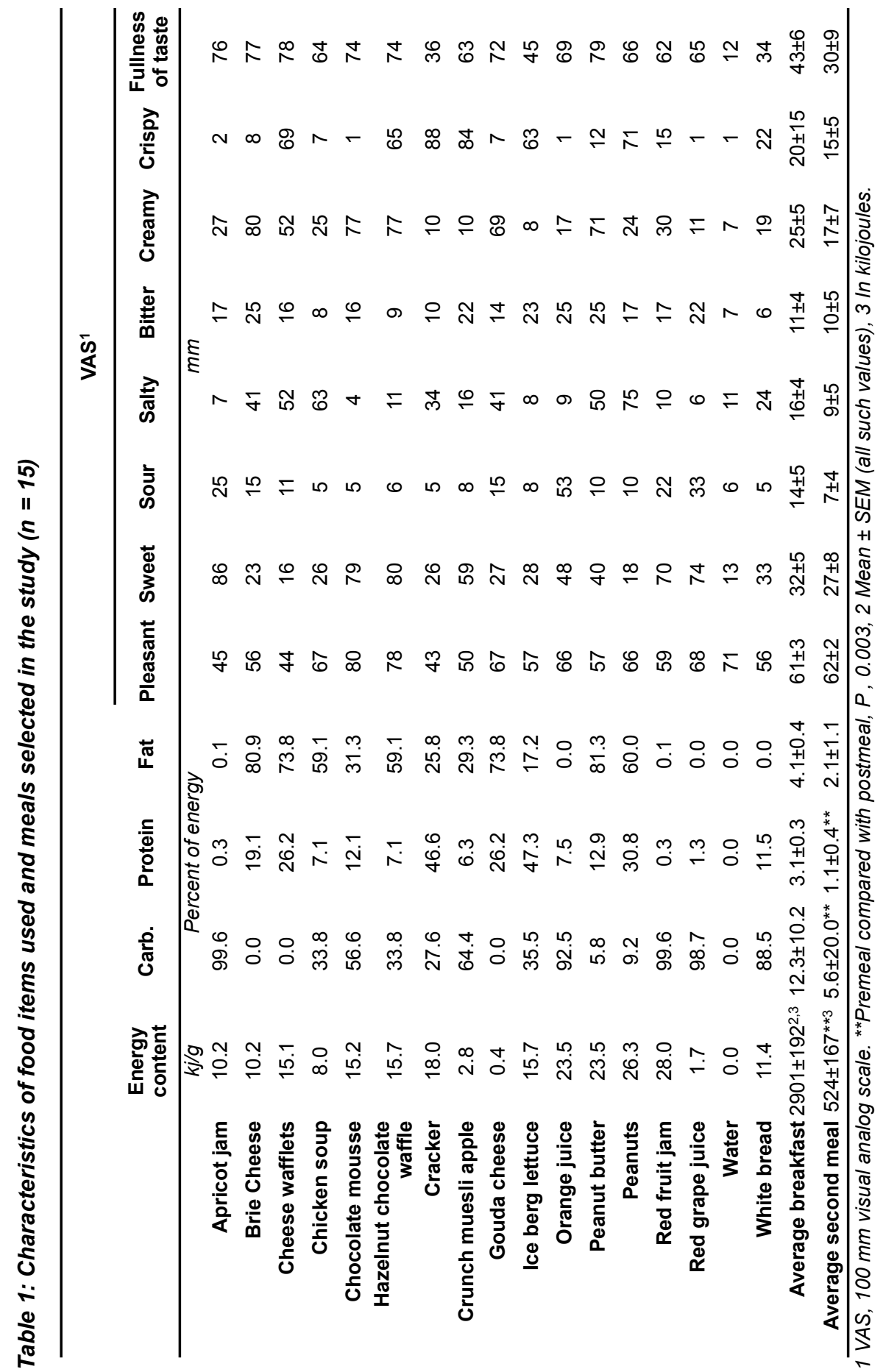

hungry are you?", "How full do you feel?", "How satiated do you feel?", "How thirsty are you?", and "How large is your desire to eat?" 


\section{Food tasting}

To assess the acceptability and familiarize subjects with all items, a food tasting test was performed on a separate day. In this session, all food items $(\mathrm{N}=17)$ were offered individually, to be tasted and rated. Water was provided to avoid cross tasting. The taste dimensions that were tested were pleasantness of taste, crispiness, creaminess, fullness of taste, sweet, sour, salty and bitter. Fullness of taste was defined by instructing the test panel to consider how strong the taste of a given food item was as well as how much this taste filled the mouth. All dimensions were rated on $100 \mathrm{~mm}$ VAS scale, anchored left with "not at all" and anchored right with "extremely" for each food item and each dimension. Group average ratings (between subjects) were calculated per food item and used in the subsequent analyses. Furthermore, group averages for macronutrient composition, total energy intake and structural characteristics of the consumed foods were calculated (Table 1).

\section{Functional MRI sessions}

To create a fasted and a satiated condition, subjects came to the university after an overnight fast of at least 10 hours: Subjects were instructed not to drink any alcoholic beverages the day before the test and not to eat, or drink coffee or tea after $10 \mathrm{pm}$ the evening before testing. Subjects individually confirmed adherence to these instructions. Each session included four VAS questionnaires for hunger and satiety and two fMRI scans during which the subsequent meal was chosen (Figure 1A). Breakfast, given between the first and the second fMRI scan, created the subsequent satiated condition. The second meal followed the second scan. Both meals consisted of the food items that were chosen from the 17 food images within the scanner (Table 1). The meals were offered immediately after the questionnaires and as quickly as possible after the scan. The fasted and satiated conditions were identical and therefore directly comparable.

\section{Functional MRI settings and paradigm}

The subjects were scanned in a Siemens Magnetom Allegra, with the standard onechannel head coil. The subjects' position was confirmed with T1 weighted scout images. During the functional run, subjects were presented with four segments of wanting and four segments of liking in which the stimuli were presented as an event related paradigm. The subjects knew all of the 17 items (Table 1) from the foodtasting test described above. Each segment consisted of an introduction slide, containing instructions for liking (preference without the need to eat the item), or wanting (how much the subject wanted to eat the respective item) and for giving the rating response with the button box (Figure 1B). The instruction was followed by a 12 second fixation cross. Each image (Figure 1C) was presented for $2 \mathrm{~s}$ after a short reminder ("like" and "eat now" respectively, $0.5 \mathrm{~s}$ ), was followed by a fixation cross for $2-5 \mathrm{~s}$, instruction to give the rating indicated by "*”, $1.5 \mathrm{~s}$ and finally an inter-trial interval of 7-9s. The reminder before every trial assured that subjects could discriminate between the otherwise identical liking and wanting paradigms at any time during the run. On average two trials in every segment were null-events, containing no image but showing a fixation cross instead. In total, each run lasted for approximately 35 minutes and each image was shown 4.5 times on average. Liking and wanting task related signaling (TRS) was modeled using only the image 
presentations, i.e. the task used to model liking or wanting, was viewing a food image and judging either its hedonic value, or the motivation to obtain it. Ratings were given by using a four-button response box (LUMltouch, Photon Control, Burnaby, BC, Canada) that was fixed to the right hand. The buttons represented a value of 1-4. The average ratings calculated directly after the scan and items with an average wanting rating of 2.5 or higher were offered to eat. All items were given in large quantities to account for the limited number of different items and therefore, subjects were allowed to stop eating after sampling each item.

Functional MRI images were acquired throughout the session using a T2* weighted protocol to obtain blood oxygen level dependent (BOLD) $T 2^{*}$ signal $(T R=2 \mathrm{~s}$, TE $=$ $26 \mathrm{~ms}$, Flip angle $=90^{\circ}$, matrix $=96 \times 96$, field of view $=269$ voxel size $2.8 \times 2.8 \times 2.6$ $\mathrm{mm}$, gap $0.2 \mathrm{~mm}$ ).

\section{fMRI data preprocessing}

The fMRI data were imported into BrainVoyager QX version 2.1 (Brain Innovation B.V., Maastricht, The Netherlands). To preprocess the functional data, slice scan time correction with cubic spline interpolation, motion correction with trilinear interpolated motion estimation and subsequent windowed sinc interpolation, and temporal high pass filtering with a window of 6 cycles was applied.

The functional data were aligned to each subjects' own $1 \mathrm{~mm}$ isovoxel high resolution T1 weighted anatomical scan. The auto alignment was performed using 6 parameter affine alignment and corrected manually under visual inspection, if necessary. Finally, all images were transformed into the Talairach coordinate system 32 using the standard procedure in BrainVoyager QX, resulting in a resolution of $3 \times 3 \times 3 \mathrm{~mm}$ voxels, resulting in functional voxels of $27 \mathrm{~mm}^{3}$. Statistical analyses were superimposed on a group average anatomical brain image.

\section{Region of interest definition}

Anatomical regions of interest (ROI) related to our hypotheses were determined from literature $9,10,16,17,18,19,20,21,22,23,24$ and generated manually on the 15 subject average anatomical (Talairach transformed) scan, using the Talairach atlas. If discernable, the regions were adapted to follow the region borders in our anatomical scan. The regions included the anterior cingulate cortex, amygdala, hippocampus, hypothalamus, putamen, medial and lateral globus pallidus and nucleus accumbens. To further specify these regions, a contrast of the localizer trials versus null events was created within the ROI volumes. The resulting activation maps were smoothed with a spatial Gaussian kernel with FWHM of $2.4 \mathrm{~mm}$, except for the hypothalamus and amygdala due to the small size of the activation. The newly generated areas filled most of the predefined regions, but slightly adjusted the outer limits, thereby excluding the voxels that were not at all involved in the process of liking and wanting task related signaling. These adjusted regions were subsequently used in the analyses.

\section{Behavioral measurements}

To determine effects of a pre-meal (hunger) and a post-meal state (absence of hunger), VAS for hunger and satiety were taken four times: before the first scan, immediately after the first scan before breakfast, before the second scan and immediately after the second scan before the second meal. The model that was 
used for the rewarding value of food consists of two elements i.e. liking and wanting. Thus, to assess the behavioral aspect of rewarding value of food and food intake, behavioral liking and wanting were analyzed using the ratings given in the scanner. Here, the question to determine liking was "how much do you like this item, not considering if you want to eat it right now?". Wanting was determined using the question "how much do you want to eat this item right now?", while the subjects were instructed that the items that were rated high in wanting were to be consumed immediately after the scan. Behavioral liking was thereby defined as explicit food preference and behavioral wanting was defined as explicit food choice.

\section{Data analysis}

To analyze the brain activation, separate predictors for liking and wanting were created, to be used in general linear model analyses and subsequent second level analyses: Subjects rated the images for all items, thus each image was ranked according to the average rating given for the respective item. From this ranking, the five highest and five lowest ranked images were selected to avoid an unbalanced design. Consequently, the predictors obtained were 'low liking', 'liking', 'low wanting' and 'wanting'. Two groups of non-selected trials that remained in between the high and low rated images, were subsequently used to adjust the size and shape of the regions of interest i.e. as localizer trials. The onset and duration of each trial were defined as the onset and duration of the image presentation and the trials were modeled using the standard canonical two-gamma hemodynamic response function. To account for habituation effects, linear habituation predictors were added using the BVA-Predictor tool (BVA-Predictor Tool, J.M. Born, Maastricht). To perform group contrasts comparing the pre-meal and post-meal conditions, dummy coding was

\section{Table 2: Behavioral correlations}

\begin{tabular}{|c|c|c|c|c|c|c|c|c|c|c|}
\hline & BMI & $\begin{array}{l}\text { Energy } \\
\text { intake } 1\end{array}$ & $\begin{array}{l}\text { Energy } \\
\text { intake } 2 \\
\end{array}$ & $\begin{array}{c}\text { Dietary } \\
\text { restrain } \\
t\end{array}$ & $\begin{array}{c}\text { Liking } \\
\text { pre- } \\
\text { meal } \\
\end{array}$ & $\begin{array}{l}\text { Liking } \\
\text { post- } \\
\text { meal } \\
\end{array}$ & $\begin{array}{c}\text { Wantin } \\
\text { g pre- } \\
\text { meal }\end{array}$ & $\begin{array}{c}\text { Wantin } \\
\text { g post- } \\
\text { meal }\end{array}$ & $\begin{array}{l}\text { Change } \\
\text { in liking }\end{array}$ & $\begin{array}{c}\text { Change } \\
\text { in } \\
\text { g wanting }\end{array}$ \\
\hline BMI & 1 & 0.023 & 0.504 & 0.079 & -0.202 & -0.006 & -0.279 & -0.31 & -0.254 & 0.164 \\
\hline $\begin{array}{r}\text { Energy intake } \\
1\end{array}$ & 0.023 & 1 & -0.076 & 0.106 & 0.293 & -0.160 & -0.029 & $-0.631^{*}$ & $0.535^{*}$ & $0.710^{* *}$ \\
\hline $\begin{array}{r}\text { Energy intake } \\
2\end{array}$ & 0.504 & -0.076 & 1 & 0.042 & 0.136 & 0.255 & -0.042 & -0.316 & -0.077 & 0.338 \\
\hline $\begin{array}{r}\text { Dietary } \\
\text { restraint }\end{array}$ & 0.079 & 0.106 & 0.042 & 1 & -0.503 & -0.333 & 0.011 & 0.142 & -0.318 & -0.157 \\
\hline $\begin{array}{r}\text { Liking pre- } \\
\text { meal }\end{array}$ & -0.202 & 0.293 & 0.136 & -0.503 & 1 & $0.642^{* *}$ & 0.216 & -0.148 & $0.652^{* *}$ & 0.324 \\
\hline $\begin{array}{r}\text { Liking post- } \\
\text { meal }\end{array}$ & -0.006 & -0.160 & 0.255 & -0.333 & $0.642^{* *}$ & 1 & 0.404 & 0.228 & -0.163 & 0.02 \\
\hline $\begin{array}{r}\text { Wanting pre- } \\
\text { meal }\end{array}$ & -0.279 & -0.029 & -0.042 & 0.011 & 0.216 & 0.404 & 1 & $0.516^{*}$ & -0.122 & 0.105 \\
\hline $\begin{array}{r}\text { Wanting post- } \\
\text { meal }\end{array}$ & -0.31 & $-0.631^{*}$ & -0.316 & 0.142 & -0.148 & 0.228 & $0.516^{*}$ & 1 & -0.416 & $-0.798^{* *}$ \\
\hline $\begin{array}{r}\text { Change in } \\
\text { liking }\end{array}$ & -0.254 & $0.535^{\star}$ & -0.077 & -0.318 & $0.652^{* *}$ & -0.163 & -0.122 & -0.416 & 1 & 0.397 \\
\hline $\begin{array}{r}\text { Change in } \\
\text { wanting }\end{array}$ & 0.164 & $0.710^{* *}$ & 0.338 & -0.157 & 0.324 & 0.02 & 0.105 & $-0.798^{* *}$ & 0.397 & 1 \\
\hline
\end{tabular}

$N=15 .{ }^{* * *}$ Correlation is significant at the 0.001 level (2-tailed) ${ }^{* *}$ Correlation is significant at the 0.01 level (2-tailed). ${ }^{*}$ Correlation is significant at the 0.05 level (2-tailed). 
applied using the BVA-Predictor tool. Group effects were determined by second level random effects analysis (ANOVA), which was performed using all GLM factors. Relevant group contrasts were high liking compared with low liking and high wanting compared with low wanting. To compare the change in liking and wanting over the meal, activation maps for liking pre-meal - liking post-meal, wanting pre-meal wanting post-meal were created and tested vs. zero separately. Overlays were created containing the liking data in light and dark blue and the wanting data in red/ orange. Significant voxels after false discovery rate (FDR) correction $(q<0.05)$ and in double overlays uncorrected $(p<0.0063)$ were used to compare activations in whole brain images and an additional functional voxel cluster threshold of $n=4 \times 27 \mathrm{~mm} 3$ was set.

To assess the balance between dietary restraint and reward seeking in relation to body-weight, neural correlates of liking and wanting were related to dietary restraint and BMI. These comparisons were executed in hungry as well as satiated conditions, while energy intake was taken into account: From regions of interest, parameter estimates for the liking and wanting models were extracted and baseline corrected, in which baseline represents the parameter estimates for the constant in the GLM/ANOVA model. Parameter estimates for high and low liking and wanting were used in GLM analysis. In the regression analyses, only the high wanting and high liking models were used. With the exception of the fMRI image data, all data were analyzed using Microsoft Excel and SPSS 16. The distribution of the data was assessed the Shapiro-Wilk test for normality. All reported data did not deviate significantly from normal distribution.

Behavioral data were analyzed using factorial ANCOVA with repeated measures. Single covariates included were none or $\mathrm{BMI}^{27,33}$. Additionally, Pearson correlations were calculated between BMI, dietary restraint, liking and wanting rating.

Pearson correlations between fMRI ROI activation data (related to high liking or high wanting) and behavioral as well as anthropometric data (hunger, energy intake, BMI, TFEQ F1) were calculated per condition (fasted pre-meal and satiated post-meal). ROI data were further assessed, using second level group analyses, by grouping test values (Pearson $r$ ) from multiple relevant regions and testing against zero using a two-sided t-test. Following our hypotheses, this was performed for the striatum related to energy intake, BMI and dietary restraint (caudate, lateral and medial globus pallidus putamen, bilaterally i.e. 8 regions pre-meal and post-meal), for the hypothalamus related to hunger and BMI (bilateral pre-meal and post-meal) and in the amygdala related to dietary restraint (bilateral pre-meal and post-meal). Values are reported as average $r \pm S E M$ with significance level. Finally a correlation analysis between dietary restraint and change in liking and wanting task related activation specifically in the nucleus accumbens was made. These relations are reported with Bonferroni correction for testing twice (bilateral).

\section{Results}

\section{Hunger, satiety and energy intake}

The fasted state before the first scan was confirmed by low VAS scores for satiety and fullness, and high scores for hunger. Eating the self-selected breakfast after the first scan lead to significantly decreased hunger (Figure 2A, $p<0.001$ ), and to 
A

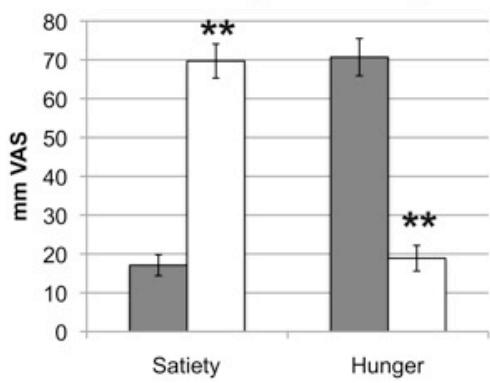

B Energy intake

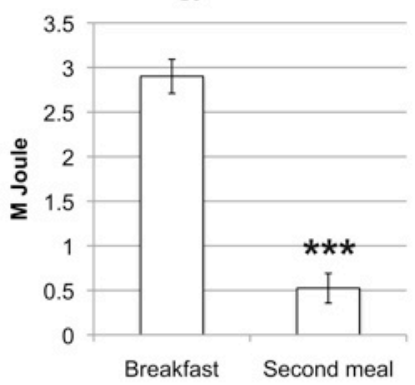

C

aPre-meal

口Post-meal

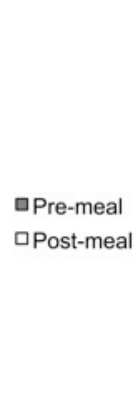

Coincidence of liking with wanting

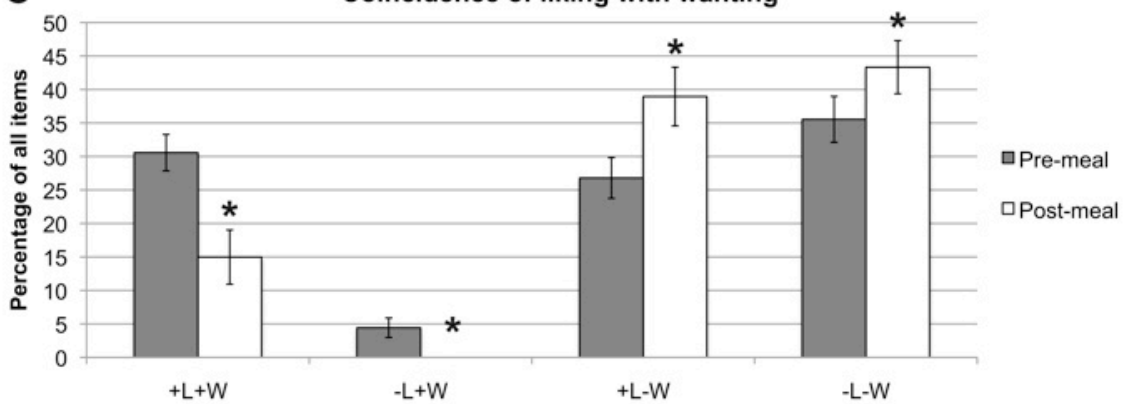

D First meal to second meal change in liking and wanting ratings
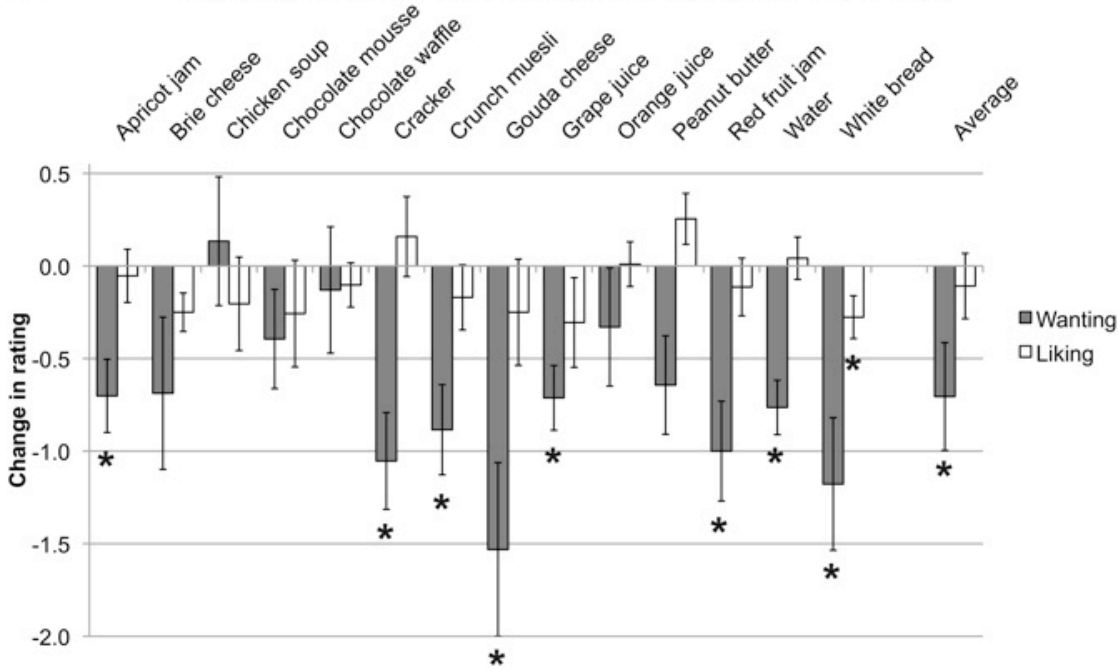

\section{Figure 2:}

Group-average ( $\pm S E M)$ values of anthropometric and behavioral data $(n=15)$. A: Hunger and satiety premeal compared with postmeal. B: Energy intake at the first and second meal. $C$ : Percentages of items with coincidence different levels of liking and wanting, in which $+L$ $+W$ denotes high liking and high wanting, $-L+W$ denotes low liking and high wanting, $+L-W$ denotes high liking and low wanting, and $-L-W$ denotes low liking and wanting. Depicted is the situation while choosing breakfast (premeal) and while choosing the second meal (postmeal). D: Average change for liking and wanting rating per item from premeal to postmeal. ${ }^{*}, * *{ }^{* *}$ Differences premeal to postmeal were significant as follows (t tests): ${ }^{*} P$, $0.05,{ }^{* *} P, 0.01,{ }^{* * *} P, 0.001$. VAS, visual analog scale. 

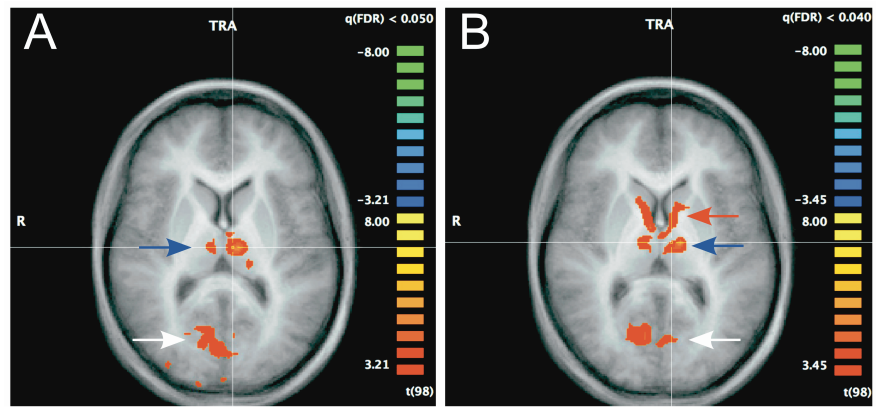

Figure 3:
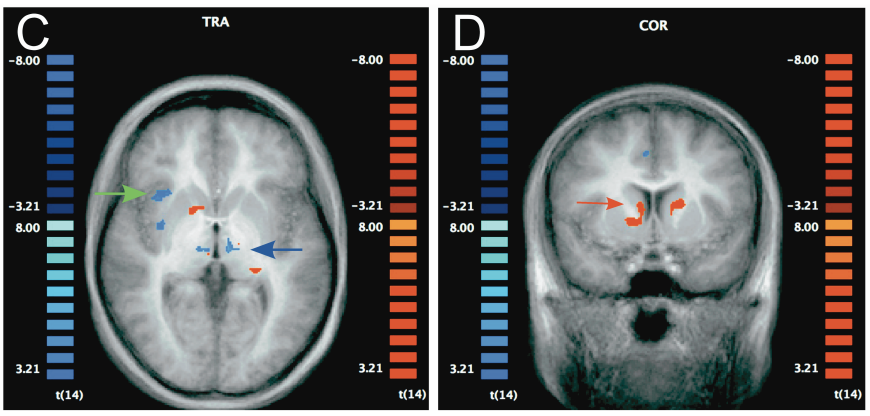

Group-average brainactivation contrasts $(n=15)$ for high liking compared with low liking [false discovery rate (FDR) $q<0.05]$ (A) and high wanting compared with low wanting (FDR q<0.04) (B). $C$ and $D$ : Overlay of premeal compared with postmeal liking (dark and light blue) and premeal compared with postmeal wanting (red and orange); both contrasts, $P<0.0063$ uncorrected). The observed effect was significant in the anterior insula for liking (C) and in the caudate for wanting (D). Arrows indicate the caudate (red), anterior insula (green), thalamus (blue), and visual cortex (white). TRA, transversal; COR, coronal; Bonf, Bonferroni corrected.

increased satiety (Figure $2 \mathrm{~A}, \mathrm{p}<0.001$ ) for the remainder of the session, confirming that our intended fasted pre-meal and satiated post-meal conditions were met. During the second meal, thus when satiated, energy intake was significantly lower than during breakfast (Figure $2 \mathrm{~B}$ and Table 1, $\mathrm{p}<0.001$ ). No changes in other average food characteristics of the selected foods were observed (Table 1, n.s.).

\section{Liking and wanting}

Behaviorally, items that were highly liked (average rating $\geq 2.5$ ) were wanted more pre-meal than post meal, while lowly liked items (average rating $<2.5$ ) were hardly chosen pre-meal $(4.4 \% \pm 1.4)$ and not at all post meal (Figure $2 \mathrm{C}$ ). From fasted to satiated conditions, average liking rating over all food items did not change significantly, while wanting ratings decreased (Figure 2D). Liking and wanting ratings were not significantly related (Table 2). Overall, wanting appeared more variable than liking between pre-meal and post-meal conditions.

In the brain, liking task related signaling (TRS) was found (contrast liking high - low) pre-meal in the thalamus and occipital visual areas (Figure 3A, FDR $q<0.05$ ). Wanting TRS was observed in the thalamus, in the caudate and occipital visual areas (contrast wanting high - low, Figure 3B, FDR $q<0.04$ ). From the fasted to the satiated condition, TRS in the caudate was reduced significantly for wanting (Figure $3 C, p<0.0063$ uncorrected), while for liking the decrease was predominantly located in the anterior insula (Figure 3D, $p<0.0063$ uncorrected). Thus overlapping TRS was found (Thalamus and visual cortex) as well as distinct regions (caudate or anterior insula), underlining the difference in TRS for liking and wanting.

In regions of interest, overall TRS pre-meal was higher than TRS post-meal in the anterior cingulate cortex, caudate, putamen, hypothalamus and lateral globus 
Table 3: Region of interest ANOVA and ANCOVA with liking low. liking high. wanting low and wanting high.

\begin{tabular}{|c|c|c|c|c|c|c|c|}
\hline Region & Effect & $\begin{array}{c}\text { Value } \\
\text { without } \\
\text { covariate }\end{array}$ & $F(1.14)$ & Sig & $\begin{array}{c}\text { Value with } \\
\text { BMI }\end{array}$ & $F(1.13)$ & Sig \\
\hline \multicolumn{8}{|c|}{ Left caudate } \\
\hline & Time & 0.313 & 6.383 & 0.024 & 0.017 & 0.222 & 0.645 \\
\hline & Question-type & 0.045 & 0.654 & 0.432 & 0.085 & 1.204 & 0.292 \\
\hline & Rating & 0.189 & 3.252 & 0.093 & 0.012 & 0.153 & 0.702 \\
\hline & $\begin{array}{r}\text { Question-type x } \\
\text { Rating }\end{array}$ & 0.070 & 1.054 & 0.322 & 0.145 & 2.211 & 0.161 \\
\hline \multicolumn{8}{|c|}{ Right caudate } \\
\hline & Time & 0.291 & 5.743 & 0.031 & 0.000 & 0.004 & 0.949 \\
\hline & Question-type & 0.002 & 0.025 & 0.876 & 0.006 & 0.084 & 0.776 \\
\hline & Rating & 0.272 & 5.244 & 0.038 & 0.069 & 0.963 & 0.344 \\
\hline & $\begin{array}{r}\text { Question-type x } \\
\text { Rating }\end{array}$ & 0.012 & 0.163 & 0.692 & 0.135 & 2.032 & 0.178 \\
\hline \multicolumn{8}{|c|}{ Anterior cingulate cortex } \\
\hline & Time & 0.484 & 13.150 & 0.003 & 0.007 & 0.095 & 0.763 \\
\hline & Question-type & 0.000 & 0.006 & 0.938 & 0.122 & 1.800 & 0.203 \\
\hline & Rating & 0.290 & 5.705 & 0.032 & 0.236 & 4.009 & 0.067 \\
\hline & $\begin{array}{r}\text { Question-type x } \\
\text { Rating }\end{array}$ & 0.002 & 0.027 & 0.872 & 0.027 & 0.356 & 0.561 \\
\hline \multicolumn{8}{|c|}{ Posterior cingulate cortex } \\
\hline & Time & 0.164 & 2.750 & 0.119 & 0.000 & 0.001 & 0.973 \\
\hline & Question-type & 0.000 & 0.006 & 0.941 & 0.278 & 4.994 & 0.044 \\
\hline & Rating & 0.088 & 1.347 & 0.265 & 0.009 & 0.115 & 0.740 \\
\hline & $\begin{array}{r}\text { Question-type x } \\
\text { Rating }\end{array}$ & 0.007 & 0.104 & 0.752 & 0.009 & 0.119 & 0.735 \\
\hline \multicolumn{8}{|c|}{ Left hypothalamus } \\
\hline & Time & 0.265 & 5.054 & 0.041 & 0.057 & 0.787 & 0.391 \\
\hline & Question-type & 0.088 & 1.355 & 0.264 & 0.038 & 0.520 & 0.484 \\
\hline & Rating & 0.225 & 4.064 & 0.063 & 0.022 & 0.299 & 0.594 \\
\hline & $\begin{array}{r}\text { Question-type x } \\
\text { Rating }\end{array}$ & 0.243 & 4.491 & 0.052 & 0.016 & 0.213 & 0.652 \\
\hline \multicolumn{8}{|c|}{ Right hypothalamus } \\
\hline & Time & 0.357 & 7.759 & 0.015 & 0.000 & 0.001 & 0.975 \\
\hline & Question-type & 0.000 & 0.004 & 0.953 & 0.004 & 0.057 & 0.815 \\
\hline & Rating & 0.166 & 2.786 & 0.117 & 0.001 & 0.009 & 0.925 \\
\hline & $\begin{array}{r}\text { Question-type x } \\
\text { Rating }\end{array}$ & 0.015 & 0.218 & 0.648 & 0.001 & 0.008 & 0.928 \\
\hline \multicolumn{8}{|c|}{ Left lateral globus pallidus } \\
\hline & Time & 0.233 & 4.263 & 0.058 & 0.042 & 0.565 & 0.466 \\
\hline & Question-type & 0.030 & 0.435 & 0.520 & 0.140 & 2.124 & 0.169 \\
\hline & Rating & 0.096 & 1.491 & 0.242 & 0.074 & 1.042 & 0.326 \\
\hline & $\begin{array}{r}\text { Question-type x } \\
\text { Rating }\end{array}$ & 0.002 & 0.027 & 0.871 & 0.342 & 6.745 & 0.022 \\
\hline
\end{tabular}




\begin{tabular}{|c|c|c|c|c|c|c|c|}
\hline Region & Effect & $\begin{array}{c}\text { Value } \\
\text { without } \\
\text { covariate }\end{array}$ & $F(1.14)$ & Sig & $\begin{array}{c}\text { Value with } \\
\text { BMI }\end{array}$ & $F(1.13)$ & Sig \\
\hline \multicolumn{8}{|c|}{$\begin{array}{l}\text { Right lateral globus } \\
\text { pallidus }\end{array}$} \\
\hline & Time & 0.266 & 5.071 & 0.041 & 0.178 & 2.810 & 0.118 \\
\hline & Question-type & 0.005 & 0.076 & 0.787 & 0.002 & 0.032 & 0.860 \\
\hline & Rating & 0.124 & 1.982 & 0.181 & 0.091 & 1.303 & 0.274 \\
\hline & $\begin{array}{r}\text { Question-type } x \\
\text { Rating }\end{array}$ & 0.056 & 0.831 & 0.378 & 0.303 & 5.665 & 0.033 \\
\hline \multicolumn{8}{|c|}{ Left medial globus pallidus } \\
\hline & Time & 0.135 & 2.176 & 0.162 & 0.005 & 0.069 & 0.797 \\
\hline & Question-type & 0.007 & 0.095 & 0.762 & 0.000 & 0.002 & 0.965 \\
\hline & Rating & 0.078 & 1.184 & 0.295 & 0.035 & 0.467 & 0.506 \\
\hline & $\begin{array}{r}\text { Question-type } x \\
\text { Rating }\end{array}$ & 0.000 & 0.002 & 0.962 & 0.268 & 4.758 & 0.048 \\
\hline \multicolumn{8}{|c|}{$\begin{array}{l}\text { Right medial globus } \\
\text { pallidus }\end{array}$} \\
\hline & Time & 0.241 & 4.436 & 0.054 & 0.057 & 0.786 & 0.392 \\
\hline & Question-type & 0.004 & 0.059 & 0.812 & 0.001 & 0.018 & 0.895 \\
\hline & Rating & 0.119 & 1.885 & 0.191 & 0.049 & 0.670 & 0.428 \\
\hline & $\begin{array}{r}\text { Question-type } x \\
\text { Rating }\end{array}$ & 0.001 & 0.021 & 0.887 & 0.309 & 5.811 & 0.031 \\
\hline \multicolumn{8}{|c|}{ Left posterior insula } \\
\hline & Time & 0.004 & 0.059 & 0.811 & 0.002 & 0.028 & 0.870 \\
\hline & Question-type & 0.234 & 4.270 & 0.058 & 0.281 & 5.083 & 0.042 \\
\hline & Rating & 0.003 & 0.045 & 0.835 & 0.080 & 1.124 & 0.308 \\
\hline & $\begin{array}{r}\text { Question-type } x \\
\text { Rating }\end{array}$ & 0.028 & 0.408 & 0.533 & 0.001 & 0.015 & 0.904 \\
\hline \multicolumn{8}{|c|}{ Right posterior insula } \\
\hline & Time & 0.026 & 0.370 & 0.553 & 0.059 & 0.818 & 0.382 \\
\hline & Question-type & 0.324 & 6.711 & 0.021 & 0.358 & 7.250 & 0.018 \\
\hline & Rating & 0.041 & 0.592 & 0.454 & 0.097 & 1.398 & 0.258 \\
\hline & $\begin{array}{r}\text { Question-type x } \\
\text { Rating }\end{array}$ & 0.002 & 0.022 & 0.885 & 0.005 & 0.068 & 0.798 \\
\hline \multicolumn{8}{|c|}{ Left putamen } \\
\hline & Time & 0.279 & 5.422 & 0.035 & 0.025 & 0.329 & 0.576 \\
\hline & Question-type & 0.018 & 0.256 & 0.621 & 0.167 & 2.607 & 0.130 \\
\hline & Rating & 0.114 & 1.798 & 0.201 & 0.036 & 0.487 & 0.498 \\
\hline & $\begin{array}{r}\text { Question-type } x \\
\text { Rating }\end{array}$ & 0.008 & 0.107 & 0.749 & 0.445 & 10.420 & 0.007 \\
\hline \multicolumn{8}{|c|}{ Right putamen } \\
\hline & Time & 0.395 & 9.152 & 0.009 & 0.000 & 0.005 & 0.942 \\
\hline & Question-type & 0.010 & 0.134 & 0.719 & 0.029 & 0.390 & 0.543 \\
\hline & Rating & 0.209 & 3.703 & 0.075 & 0.048 & 0.651 & 0.434 \\
\hline & $\begin{array}{r}\text { Question-type } x \\
\text { Rating }\end{array}$ & 0.001 & 0.019 & 0.891 & 0.369 & 7.610 & 0.016 \\
\hline
\end{tabular}

Tests were performed in 18 regions of interest (14 regions are shown). Excluded regions were the left and right hippocampus and the left and right thalamus. Time, pre-meal or post-meal; Question type, liking or wanting, Rating, high or low. ' $x$ ' denotes interaction effect. 
pallidus (Table 3, all $\mathrm{p}<0.05$ ). When corrected for BMI, liking TRS resulted in higher activation than wanting TRS in the posterior insula and the posterior cingulate cortex (Table 3, question-type effect, i.e. liking vs. wanting, liking > wanting, all $p<0.05$ ). A significant interaction effect of question-type and rating (i.e. high vs. low) was found in the hypothalamus, globus pallidus and putamen (Table 3 , all $p<0.05$ ). Thus
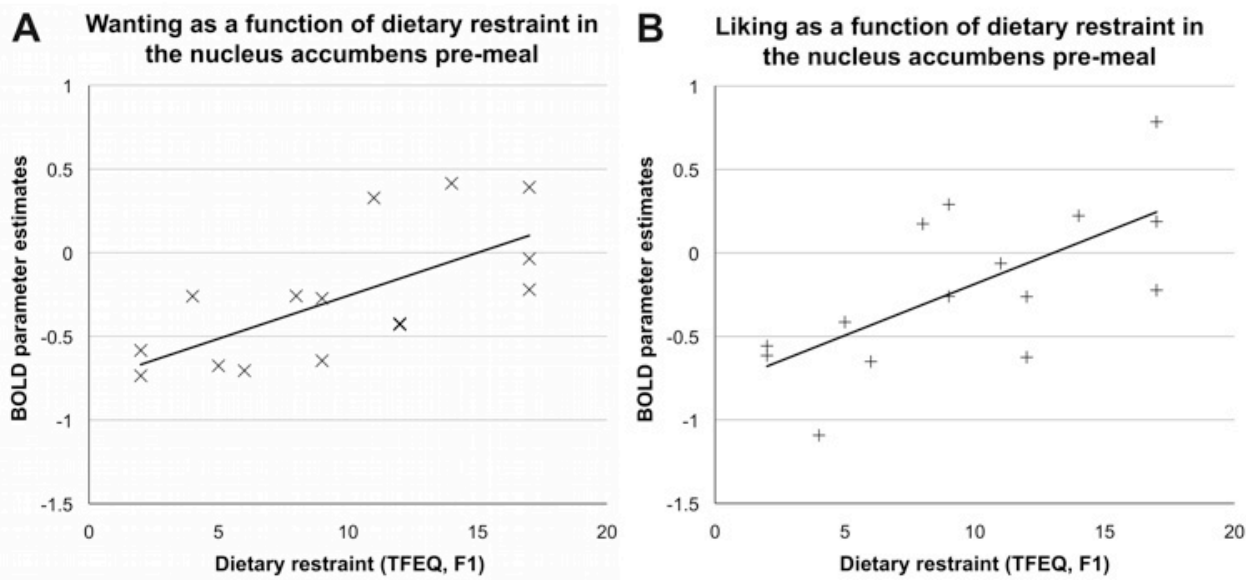

C Change in liking signaling as a function of dietary restraint in the nucleus accumbens

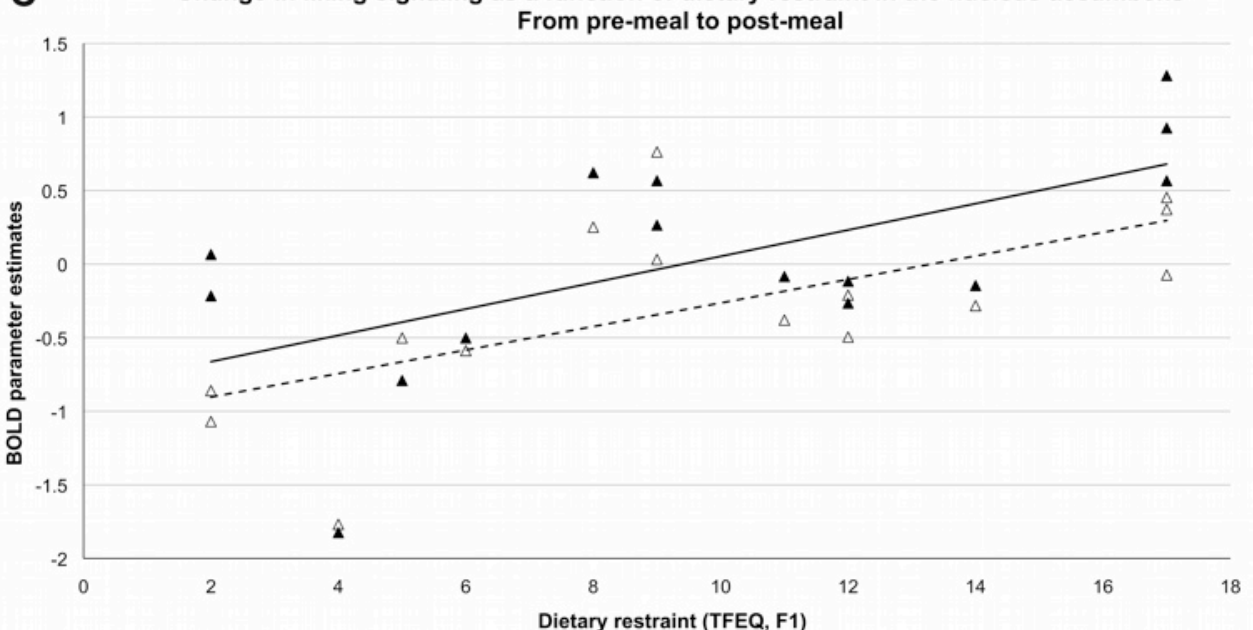

\ Left nucleus accumbens (NAc)

$\triangle$ right NAC -Left NAC - - right NAC

\section{Figure 4:}

Wanting $(A)$ and liking $(B)$ task-related signaling in the nucleus accumbens (NAC) as a function of dietary restraint [Three-Factor Eating Questionnaire (TFEQ), F1; $n=15]$. Lines represent trends, wanting task-related signaling is denoted by ".", and liking task-related signaling by "+", each representing a single subject. Trends shown were significant at $P<0.05$ (left NAc). C: The change in liking task-related signaling from premeal to postmeal in the NAc related to dietary restraint. Change in liking task-related signaling per subject is represented by triangles. Trends shown were significant at $P<0.04$ (Bonferroni corrected for bilateral testing). The solid line denotes the left NAc, and the dotted line denotes the right NAc. BOLD, blood oxygen level dependent. 
specific activation related to the liking task was located in the posterior insula and wanting in the striatum and cingulate cortex, and secondly, the variability in liking and wanting TRS was related to BMI.

\section{Liking and wanting TRS in relation to cognitive dietary restraint}

Regarding our second hypothesis, liking TRS post-meal was inversely related to cognitive dietary restraint in the left thalamus, cingulate cortex, amygdala and most of the striatum (smallest $R^{2}(13)=0.26, p<0.05$, liking TRS, post-meal, second level group analysis $r=-0.493 \pm 0.030, p<0.00001$, data not shown).

Liking and wanting TRS in the left nucleus accumbens correlated positively to dietary restraint before eating breakfast (Figure $4 A$ and $B$, smallest $R^{2}(13)=0.27$, $p<0.05)$. The change in liking TRS in left and right the nucleus accumbens from pre-
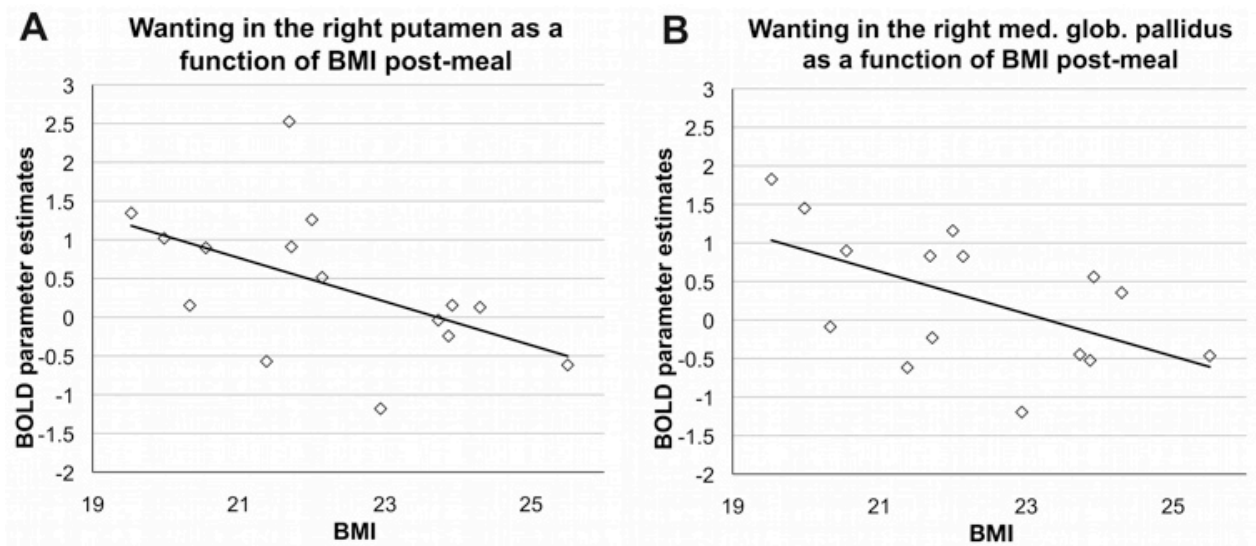

C Wanting in the right hypothalamus as a function of BMI post-meal
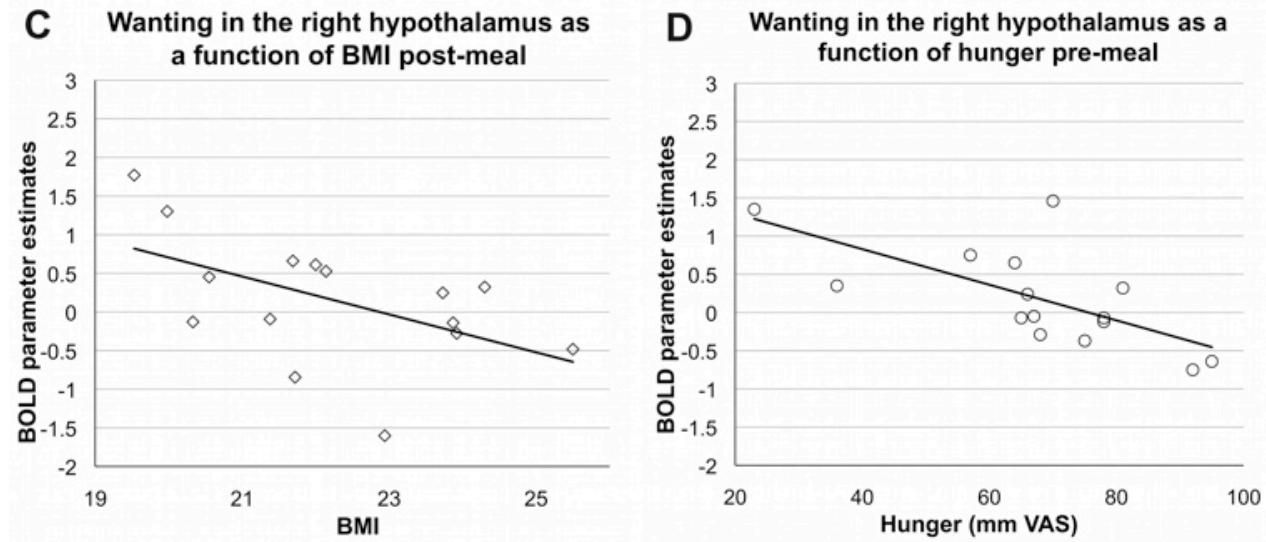

\section{Figure 5:}

Wanting task-related signaling in the striatum as a function of BMI is represented by one diamond per subject. Data are shown for the putamen $(A)$ and right medial globus (glob.) pallidus (B). C: Wanting task-related signaling in the right hypothalamic region as a function of $B M I$ is represented as one circle per subject. D: Wanting task-related signaling in the hypothalamic region as a function of hunger. Trends shown were significant at $P<0.05(n=15)$. Overall, BMI was a function of wanting task-related signaling in the striatum (grouped caudate, putamen, and globus pallidus): $P<0.00002$. BOLD, blood oxygen level dependent; VAS, visual analog scale. 
meal to post-meal was a function of dietary restraint (Figure $4 C$, smallest $R^{2}(13)$ $=0.36$, Bonferroni corrected $p<0.04$ ).

Effectively, in subjects with high dietary restraint compared with subjects with low dietary restraint, the rewarding value of food (the combination of liking and wanting), was lower after eating, while it was higher for those subjects when fasted.

\section{Liking and wanting TRS in energy homeostasis}

Correlations were made between liking or wanting TRS and BMI. Post-meal, i.e. in the satiated state, wanting activation in the putamen, medial globus pallidus and hypothalamus was an inverse function of BMI (Figure 5A, B and C). Using second level analysis on these relationships, an overall negative relation between BMI and wanting TRS in the striatum $(r=-0.293 \pm 0.036, p<0.00002)$ and hypothalamus $(r=-0.391 \pm 0.069, p=0.011$, data not shown) were found. Thus subjects with a higher BMI exhibited lower liking and wanting TRS, i.e. lower rewarding value of food, in multiple brain regions.

Moreover, liking or wanting TRS specifically in the hypothalamus, were correlated to hunger ratings in accordance with our hypotheses. Pre-meal, wanting TRS in the hypothalamus was an inverse function of hunger (Figure 5D), post-meal this was not significant, thus TRS in the hypothalamus was dependent on the energy state.

The link between energy balance and TRS was further analyzed: Post-meal, liking TRS in the right putamen, anterior insula and wanting TRS in the hypothalamus and right globus pallidus were inversely related to energy intake during the second meal (smallest $\left.R^{2}(13)=0.26 p<0.05\right)$. Using second level group analysis, we observed an inverse relationship between liking and wanting TRS in the striatum and energy intake during both meals (liking $r=-0.284 \pm 0.052, p<0.0001$ and wanting $r=-0.306 \pm 0.033, p<0.00001$, data not shown); high energy intakes were related with low TRS for liking and wanting, i.e. the rewarding value of food.

\section{Discussion}

\section{Hunger, satiety and energy intake}

This study targeted the difference in brain signaling between the fasted state and the satiated state. Upon arrival, subjects showed high hunger and low satiety, while post meal, hunger was decreased and satiety was increased significantly and the differences were of considerable magnitude (Figure 2A, Table 1). When satiated, significantly less food was chosen, resulting in lower energy intake (Figure 2B). These findings confirmed the presence of pre-meal fasted 'hunger' conditions and the post-meal 'satiated' conditions.

\section{Liking and wanting}

Behaviorally, liking and wanting was assessed by image ratings for liking and wanting, i.e. as an explicit measure. We found that subjects were able to distinguish liking and wanting: Wanting ratings were significantly reduced over breakfast, while liking ratings did not change (Figure 2D). Furthermore, these findings agree with our earlier studies, in which we showed greater stability of liking compared with wanting ${ }^{13,17}$, indicating that our simplified fMRI optimized liking and wanting paradigm performed appropriately to distinguish the two concepts. 
For the first time, we compared both liking and wanting task related signaling (TRS) in the human brain using a comparable method for liking and wanting. This is in contrast with earlier studies that assessed either liking or wanting separately $24,33,34$, or used different paradigms for liking and wanting in rodents ${ }^{10,35}$. In our group, liking and wanting TRS was seen in overlapping areas, independent of the fasted or satiated condition (Thalamus and visual areas, Figure 3). Moreover, liking TRS was found specifically in the anterior insula and wanting TRS in the caudate (Figure 3). Overlap as well as distinctiveness of liking and wanting were further confirmed by region of interest analyses: liking and wanting TRS were found in the nucleus accumbens (Figure 4), wanting but not liking TRS was found in the cingulate cortex, thalamus and striatum (caudate, putamen, medial and lateral globus pallidus, Figure 5 , and Table 3), while liking TRS was found in the anterior and posterior insula (Figure $3 \mathrm{C}$, Table 3). These finding are in line with our hypotheses and underline the model of the rewarding value: liking and wanting are signaled as two distinct aspects of one overall process ${ }^{10,36}$, i.e. they are signaled in some overlapping and some specific regions.

\section{Liking and wanting signaling in relation to cognitive dietary restraint}

We established a novel relation between dietary restraint and liking and wanting TRS in the nucleus accumbens (Figure 4), a key area in signaling of reward $16,36,37$. Here, high dietary restraint was related to high liking and wanting TRS pre-meal. Thus in the fasted condition the rewarding value of food predicted the level of dietary restraint, in line with studies showing an increased reinforcing value of items temporarily omitted from the diet 38,39 . Over the meal we observed that the liking specific component of reward decreased in the nucleus accumbens as a function of dietary restraint. Thus during successful dietary restraint, represented in our subject group ${ }^{6,7}$, lower liking TRS, specifically in the nucleus accumbens post-meal, leads to decreased rewarding value of food ${ }^{36}$. Thereby, our data suggest, that cognitive dietary restraint may increase the rewarding value of food pre-meal in order to reach reward balance during eating at an appropriate time, thus avoiding reward deficiency ${ }^{28}$, while post-meal decreasing reward-related signaling to decrease eating motivation, similar to the reward sensitivity theory ${ }^{25}$.

\section{Liking and wanting TRS indicating reward deficiency}

Energy intake and post-meal liking and wanting TRS in the striatum and cingulate cortex were inversely related post-meal, suggesting that food reward-related signaling is an important factor in eating while satiated. Moreover, we showed that energy intake is generally inversely related to liking and wanting TRS in the striatum. At the same time, subjects with higher BMl exhibited lower wanting TRS in striatum, hypothalamus and anterior insula (Figure 5A-B, D). Thus all findings are in line with the reward deficiency $27,28,40$ : lower reward-related signaling was related to higher energy intakes in our subjects. At the same time, lower reward-related signaling was associated with higher BMI. While we found support for the reward deficiency syndrome in our normal weight subjects $(B M I=19.5-25.5)$, it is possible that the reward sensitivity theory applies to subjects with $\mathrm{BMI}<19$ : a study indicating reward sensitivity in humans included subjects in the below normal and low normal range of BMI 26. 


\section{Liking and wanting related signaling in energy homeostasis}

Pre-meal but not post-meal, wanting TRS in the hypothalamus was an inverse function of hunger (Figure 5C), i.e. hypothalamus activation was dependent on energy state. This is in agreement with the role of the hypothalamus as the regulatory region in homeostasis ${ }^{35,41}$, suggesting adequacy of our paradigm to measure explicit wanting related processes: While choosing food in the fasted state, subjects select food according to their need (homeostatic eating), which was reflected in hypothalamic signaling. When satiated, hunger was detached from hypothalamic signaling in our subjects (Figure 5B).

\section{Summary}

In the brain, liking and wanting TRS was found in some separate and some overlapping regions, confirming the uniqueness of liking and wanting while showing the close link between the two concepts that together determine the rewarding value of food. Notably, an effect of dietary restraint on this rewarding value of food was observed in the nucleus accumbens: subjects with higher dietary restraint showed higher reward-related signaling when fasted and relatively lower signaling when satiated. This implies that successful dietary restraint $28,29,42$ may express itself as a relatively highly anticipated pre-meal reward and post-meal reward suppression, representing decreased hedonic properties of food stimuli when satiated. Consequently, the motivation to obtain food reward may be decreased, as expected in the reward sensitivity theory, rather than inducing reward compensation as expected in the reward deficiency syndrome ${ }^{27,28,40}$. Thus, successful dietary restraint appears to be represented more similarly to reward sensitivity ${ }^{25,26}$.

Surprisingly, our study suggests reward deficiency even in our normal weight subject population. Energy intake and BMI were inversely related to reward-related signaling and our findings indicate that reward deficiency may have the most significant effect while satiated, which is in agreement with this theory stating that reward deficiency leads to reward seeking that may result in eating while satiated and consequently overeating $5,17,27,28,29$.

\section{Conclusion}

We confirmed the distinctiveness of liking and wanting for the first time in humans both behaviorally and in terms of the human brain regions involved. While liking was specifically represented in the insula, wanting appeared specifically in the striatum and cingulate cortex. Importantly, we found that cognitive dietary restraint predicts the change in liking TRS from pre- to post-meal in the nucleus accumbens, which sheds light on the process of successful cognitive dietary restraint. Moreover, inverse relationships of liking and wanting TRS in the striatum and BMI or energy intake support the reward deficiency hypothesis surprisingly strongly, in our normal weight subjects.

\section{Acknowledgements}

Thanks to A. Heinecke for extensive support with BrainVoyager QX. 


\section{References}

1 Seidell, J. C. Epidemiology and health economics of obesity. Medicine 34, 506-509 (2006).

2 Catenacci, V. A., Hill, J. O. \& Wyatt, H. R. The Obesity Epidemic. Clinics in Chest Medicine 30, 415-444 (2009).

3 Westerterp, K. R. \& Speakman, J. R. Physical activity energy expenditure has not declined since the 1980s and matches energy expenditures of wild mammals. Int J Obes 32, 1256-1263 (2008).

4 Pietilainen, K. H. et al. Inaccuracies in food and physical activity diaries of obese subjects: complementary evidence from doubly labeled water and co-twin assessments. Int J Obes 34, 437-445 (2010).

5 Rutters, F., Nieuwenhuizen, A. G., Lemmens, S. G. T., Born, J. M. \& Westerterp-Plantenga, M. S. Acute Stressrelated Changes in Eating in the Absence of Hunger. Obesity 17, 72-72 (2009).

6 Westerterp-Plantenga, M. S., Kempen, K. P. \& Saris, W. H. Determinants of weight maintenance in women after diet-induced weight reduction. Int J Obes Relat Metab Disord 22, 1-6 (1998).

7 Pasman, W. J., Saris, W. H. \& Westerterp-Plantenga, M. S. Predictors of weight maintenance. Obes Res 7 , 43-50 (1999).

8 Stunkard, A. J. \& Messick, S. The three-factor eating questionnaire to measure dietary restraint, disinhibition and hunger. J Psychosom Res 29, 71-83 (1985).

9 Epstein, L. H., Leddy, J. J., Temple, J. L. \& Faith, M. S. Food reinforcement and eating: a multilevel analysis. Psychological bulletin 133, 884-906 (2007).

10 Berridge, K. C. Food reward: brain substrates of wanting and liking. Neurosci Biobehav Rev 20, 1-25 (1996).

11 Finlayson, G., King, N. \& Blundell, J. The role of implicit wanting in relation to explicit liking and wanting for food: Implications for appetite control. Appetite 50, 120-127 (2008).

12 Finlayson, G., King, N. \& Blundell, J. E. Is it possible to dissociate 'liking' and 'wanting' for foods in humans? A novel experimental procedure. Physiol Behav 90, 36-42 (2007).

13 Lemmens, S. G. T. et al. Eating what you like induces a stronger decrease of 'wanting' to eat. Physiology \& Behavior 98, 318-325, doi:10.1016/j.physbeh.2009.06.008 (2009).

14 Epstein, L. H., Truesdale, R., Wojcik, A., Paluch, R. A. \& Raynor, H. A. Effects of deprivation on hedonics and reinforcing value of food. Physiology \& Behavior 78, 221-227 (2003).

15 Robinson, T. E. \& Berridge, K. C. Addiction. Annu Rev Psychol 54, 25-53 (2003).

16 Knutson, B., Adams, C. M., Fong, G. W. \& Hommer, D. Anticipation of Increasing Monetary Reward Selectively Recruits Nucleus Accumbens. J. Neurosci. 21, 159RC- (2001).

17 Born, J. M. et al. Acute stress and food-related reward activation in the brain during food choice during eating in the absence of hunger. Int J Obes 34, 172-181 (2010).

18 Blackburn, J. R., Pfaus, J. G. \& Phillips, A. G. Dopamine functions in appetitive and defensive behaviours. Prog Neurobiol 39, 247-279 (1992).

19 Murray, E. A. The amygdala, reward and emotion. Trends in cognitive sciences 11, $489-497$ (2007).

20 Schultz, W., Tremblay, L. \& Hollerman, J. R. Reward Processing in Primate Orbitofrontal Cortex and Basal Ganglia. Cereb. Cortex 10, 272-283, doi:10.1093/cercor/10.3.272 (2000).

21 Gottfried, J. A., O'Doherty, J. \& Dolan, R. J. Encoding predictive reward value in human amygdala and orbitofrontal cortex. Science 301, 1104-1107 (2003).

22 Kelley, A. E. et al. Opioid modulation of taste hedonics within the ventral striatum. Physiology \& Behavior 76, 365 (2002).

23 Kelley, A. E., Baldo, B. A., Pratt, W. E. \& Will, M. J. Corticostriatal-hypothalamic circuitry and food motivation: Integration of energy, action and reward. Physiology \& Behavior 86, 773-795 (2005).

24 Small, D. M., Jones-Gotman, M. \& Dagher, A. Feeding-induced dopamine release in dorsal striatum correlates with meal pleasantness ratings in healthy human volunteers. Neuroimage 19, 1709-1715, doi:S1053811903002532 [pii] (2003).

25 Davis, C. et al. From motivation to behaviour: a model of reward sensitivity, overeating, and food preferences in the risk profile for obesity. Appetite 48, 12-19 (2007).

26 Franken, I. H. \& Muris, P. Individual differences in reward sensitivity are related to food craving and relative body weight in healthy women. Appetite 45, 198-201, doi:S0195-6663(05)00042-5 [pii] 10.1016/j.appet.2005.04.004 (2005).

27 Wang, G. J. et al. Brain dopamine and obesity. Lancet 357, 354-357 (2001).

28 Blum, K. et al. Reward deficiency syndrome: a biogenetic model for the diagnosis and treatment of impulsive, addictive, and compulsive behaviors. J Psychoactive Drugs 32 Suppl, i-iv, 1-112 (2000).

29 Kenny, P. J. Brain reward systems and compulsive drug use. Trends Pharmacol Sci 28, 135-141, doi:S0165-6147(07)00027-2 [pii] 10.1016/j.tips.2007.01.008 (2007).

30 Koob, G. F. \& Volkow, N. D. Neurocircuitry of Addiction. Neuropsychopharmacology 35, 217-238 (2009).

31 Allison, D. B. et al. Antipsychotic-Induced Weight Gain: A Comprehensive Research Synthesis. Am J Psychiatry 156, 1686-1696 (1999).

32 Talairach, J. \& Tournoux, P. Co-planar Stereotaxic Atlas of the Human Brain. (Thieme, 1988).

33 Stice, E., Spoor, S., Bohon, C. \& Small, D. M. Relation Between Obesity and Blunted Striatal Response to Food Is Moderated by TaqIAA1 Allele. Science 322, 449-452, doi:10.1126/science.1161550 (2008).

34 Small, D. M., Zatorre, R. J., Dagher, A., Evans, A. C. \& Jones-Gotman, M. Changes in brain activity related to eating chocolate: from pleasure to aversion. Brain 124, 1720-1733 (2001). 
35 Berthoud, H. R. Neural control of appetite: cross-talk between homeostatic and non-homeostatic systems. Appetite 43, 315-317 (2004).

36 Berridge, K. C. 'Liking' and 'wanting' food rewards: Brain substrates and roles in eating disorders. Physiology \& Behavior 97, 537-550 (2009).

37 Knutson, B. \& Cooper, J. C. Functional magnetic resonance imaging of reward prediction. Current opinion in neurology 18, 411-417 (2005).

38 Goldstone, A. P. et al. Fasting biases brain reward systems towards high-calorie foods. European Joumal of Neuroscience 30, 1625-1635 (2009).

39 Temple, J. L., Chappel, A., Shalik, J., Volcy, S. \& Epstein, L. H. Daily consumption of individual snack foods decreases their reinforcing value. Eating behaviors 9, 267-276 (2008).

40 Stice, E., Yokum, S., Bohon, C., Marti, N. \& Smolen, A. Reward circuitry responsivity to food predicts future increases in body mass: Moderating effects of DRD2 and DRD4. Neuroimage 50, 1618-1625 (2010).

41 Woods, S. C. \& D'Alessio, D. A. Central control of body weight and appetite. J Clin Endocrinol Metab 93, S37-50, doi:93/11_Supplement_1/s37 [pii] 10.1210/jc.2008-1630 (2008).

42 Davis, C. \& Fox, J. Sensitivity to reward and body mass index (BMI): evidence for a non-linear relationship. Appetite 50, 43-49 (2008). 
Chapter 3

\section{A Nonlinear relationship between food choice related brain signaling in the absence of hunger and BMI}

Jurriaan M Born, Mieke JI Martens, Sofie GT Lemmens, Rainer Goebel, and Margriet S Westerterp-Plantenga, submitted for publication. 


\section{Abstract}

Background: Two models for the relation between BMI and motivational systems exist: the reward sensitivity hypothesis and the reward deficiency syndrome. Recently, it was speculated that a u-shaped relation might explain these seemingly contradictory hypotheses and unify them.

Aim: To determine the type of relation between body weight and reward-related brain signaling.

Methods: 27 female subjects $(21.6 \pm 2.2 y, B M I=25.0 \pm 3.7)$ completed two fMRI scans: one fasted; one satiated. During scans, subjects rated liking and wanting for food images, choosing those to eat immediately afterwards. Parameter estimates for wanting task related signaling were extracted and related with BMI using linear and polynomial models.

Results: Hunger changed significantly over the meal $(p<0.001)$. Behavioral wanting ratings were lower after breakfast compared with before $(p<0.001)$. Between BMI and wanting task related signaling (TRS) no linear relation was found. The best overall model, was a nonlinear relationship with a peak/nadir at $\mathrm{BMI}=24$, which was observed in the hypothalamic region and striatum (caudate and putamen). The transformed BMI model was inversely related to wanting TRS in the hypothalamic region and striatum post-meal which was statistically significant within the individual regions $\left(R^{2}>0.10, p<0.04\right)$ and the mean of these regions (hypothalamic region and striatum) was statistically different from zero $(r=-0.40 \pm 0.3, p<0.0001)$.

Thus, only with nonlinear modeling was BMI significantly related to post-meal wanting task related signaling in the hypothalamus, and the striatum indicating that subjects with BMls increasing above and decreasing below 25 exhibited progressively higher wanting task related signaling.

Conclusion: The relationship between reward-related brain signaling and BMI is nonlinear. This implies the lowest motivation may imply the lowest motivation to eat at a BMI of 25 , stimulating weight gain in thin individuals, and supporting overweight and obesity in the overweight and obese. 


\section{Introduction}

The prevalence of obesity and its co-morbidities is increasing in the developed world $^{1,2}$. This is caused by energy-intake exceeding energy expenditure, i.e. a positive energy balance ${ }^{3}$ and may be influenced by features of food intake, such as eating in the absence of hunger ${ }^{4}$. This overeating is often driven by reward seeking ${ }^{5}$. When trying to explain how eating and body weight is related to reward related signaling in the brain, two models were developed: The reward sensitivity theory, states that high reward sensitivity leads to seeking of more reward ${ }^{6,7}$, effectively maximizing the experienced reward. In contrast, the reward deficiency syndrome ${ }^{8,9}$, explains reward seeking as trying to maintain reward homeostasis while the experienced reward is reduced, thus implying that subjects with lower reward sensitivity need to increase the frequency or intensity of the rewarding activity ${ }^{10,11}$. Reward deficiency was shown as inverse relationship of dopamine receptor density and $\mathrm{BMI}$ in obese individuals ${ }^{8}$, or decreased dopaminergic reward signaling after repeated consumption of rewarding substances, such as drugs or food ${ }^{11,12}$. Furthermore, dopamine antagonists lead to an increased body weight by increasing energy-intake in many individuals ${ }^{13}$. Davis and Fox (2008) proposed a combined model, that uses an inverted u-shaped relation, such that in the lower body-weight range the reward sensitivity theory applies, while in the higher body-weight range, the reward deficiency syndrome theory applies ${ }^{14}$. This theory thus proposes that individuals with high reward sensitivity tend to have higher body weight and tend to get heavier. At a certain weight, reward deficiency may develop, leading to a further increased body weight due to compensatory reward seeking. To study the relation between the rewarding value of food and subjects characteristics, we recently used Berridge's model that describes the rewarding value of food as 'liking', the hedonic preference or palatability, and 'wanting', the motivation to eat a particular food item $^{15}$. Energy-intake is mainly determined by wanting ${ }^{5,16}$, while liking is relatively more stable ${ }^{16}$. We showed that liking and wanting are signaled in a partially overlapping and a partially distinct fashion in the human brain ${ }^{16}$. Additionally, we observed that wanting task related signaling was represented significantly in the hypothalamic region and the striatum. Signaling in the satiated condition in these regions was typically inversely related with BMI in the range of $19-25^{16}$. So far it remained unclear, how reward related signaling is affected in the BMI range of 25-33 or over all, 19-33. Here we studied the relation between BMI and wanting task related signaling in the hypothalamic region and striatum, here caudate and putamen.

We used a linear and nonlinear model to assess the relationship between BMI and brain signaling in reward and homeostasis related regions and expected to find the best fit with a nonlinear relationship, explaining the difficulty to find significant results when using a linear model.

\section{Subjects and methods}

\section{Subjects}

The study was approved and registered under MEC 09-3-035 by the Medical Ethical Committee of Maastricht University and at the Netherlands Trial Register under NTR1904. Subject recruitment started on November 23rd 2009 and included 
subjects with $\mathrm{BMI}=19-33.35$ test subjects were screened. Informed, written consent was obtained from all subjects. Inclusion criteria were: healthy female subjects, normal to slightly obese body weight (BMI 19-33) and right-handedness. Exclusion criteria were recent dieting, a personal or familial history of psychiatric disorder, or use of intra-uterine contraceptives. 30 subjects were included with the following characteristics: Age $=21.6 \pm 2.2, \mathrm{BMI}=25.0 \pm 3.7$, range 19.4-32.8. During fMRI data analyses, 3 subjects were excluded due to excessive movement, resulting in a total of 27 subjects in the subsequent analyses.

\section{Functional MRI sessions}

To create a fasted and a satiated condition, subjects came to the university after an overnight fast of at least 10 hours: Subjects were instructed not to drink any alcoholic beverages the day before the test and not to eat, or drink coffee or tea after 10 PM the evening before testing. Subjects individually confirmed adherence to these instructions. Each session included five VAS questionnaires for hunger and satiety and two fMRI scans during which the subsequent meal was chosen (Figure 1A). Breakfast, given between the first and the second fMRI scan, created the subsequent satiated condition. The second meal followed the second scan. Both meals consisted of the food items that were chosen from the 15 food images within the scanner. The meals were offered immediately after the questionnaires and as quickly as possible after the scan. The fasted and satiated conditions were identical and therefore directly comparable.

\section{Functional MRI settings and paradigm}

The subjects were scanned in a 3T Siemens Magnetom Allegra, with the standard one-channel head coil. The subjects' position was confirmed with T1 weighted scout images. During the functional run, the subjects were presented with each four segments of wanting and four segments of liking. All food items had been tasted and rated for multiple characteristics on a separate day and were thereby known to the subjects ${ }^{16}$. The items were presented one by one, asking subjects to rate the image for either 'liking' of 'wanting' (Figure 1). In total, the paradigm took approximately 35 min and each image was shown 4.5 times on average. Ratings were given by using a four-button response box (LUMItouch, Photon Control, Burnaby, BC, Canada) that was fixed to the right hand. The buttons represented a value of 1-4. The average ratings calculated directly after the scan and items with an average wanting rating of 2.5 or higher were offered to eat. All items were given in large quantities to account for the limited number of different items and therefore, each item had to be sampled at least, but not mandatorily eaten completely.

Functional MRI images were acquired throughout the session using a $\mathrm{T}^{*}$ weighted protocol to obtain blood oxygen level dependent $(B O L D) T 2^{*}$ signal $(T R=2 \mathrm{~s}, \mathrm{TE}=$ $26 \mathrm{~ms}$, Flip angle $=90^{\circ}$, matrix $=96 \times 96$, field of view $=269$ voxel size $3.0 \times 3.0 \times 2.9$ $\mathrm{mm}$, gap $0.1 \mathrm{~mm}$ ).

\section{fMRI data preprocessing}

The fMRI data were imported into BrainVoyager QX version 2.3 (Brain Innovation B.V., Maastricht, The Netherlands). Functional data was preprocessed using the standard methods in Brainvoyager QX 16,17. Functional data were aligned to each subjects' own $1 \mathrm{~mm}$ isovoxel high resolution T1 weighted anatomical scan using 
auto alignment (6 parameter affine) with supervision. Finally, all images were transformed into the Talairach coordinate system (Talairach and Tournoux, 1988) using the standard procedure in BrainVoyager $Q X$, resulting in a resolution of $3 \times 3 \times 3 \mathrm{~mm}$ voxels, resulting in a functional voxel size of $27 \mathrm{~mm}^{3}$.

\section{Region of interest definition}

Anatomical regions of interest $(\mathrm{ROI})$ related to our hypotheses were determined from our earlier study ${ }^{16}$. These ROls included the hypothalamus, which is a key region in homeostatic signaling ${ }^{16}$, the nucleus accumbens as a region in which high dopaminergic reward related signaling was observed previously ${ }^{18}$, and additionally the caudate and putamen, which are reward related areas that were consistently activated related to wanting tasks 9,16 . The ROls definitions from the previous study were used ${ }^{16}$. These definitions were based on a localizer contrast for wanting in the predefined anatomical regions, based on a group average.

\section{Behavioral measurements}

To assess the success with creating a pre-meal (hunger) and a post-meal state (absence of hunger), VAS for hunger and satiety were taken four times: before the first scan, immediately after the first scan, before the second scan and immediately after the second scan. The model that was used for the rewarding value of food consists of two elements i.e. liking and wanting. Thus to assess the rewarding value

A

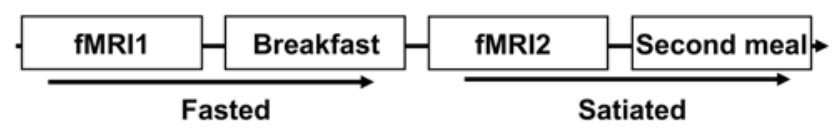

c
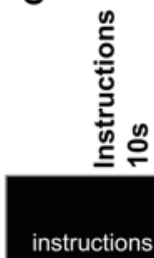

instructions

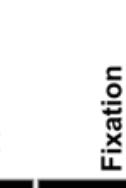

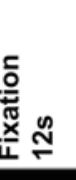
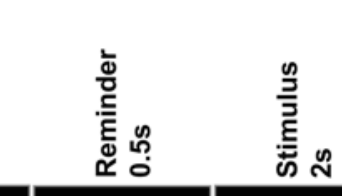
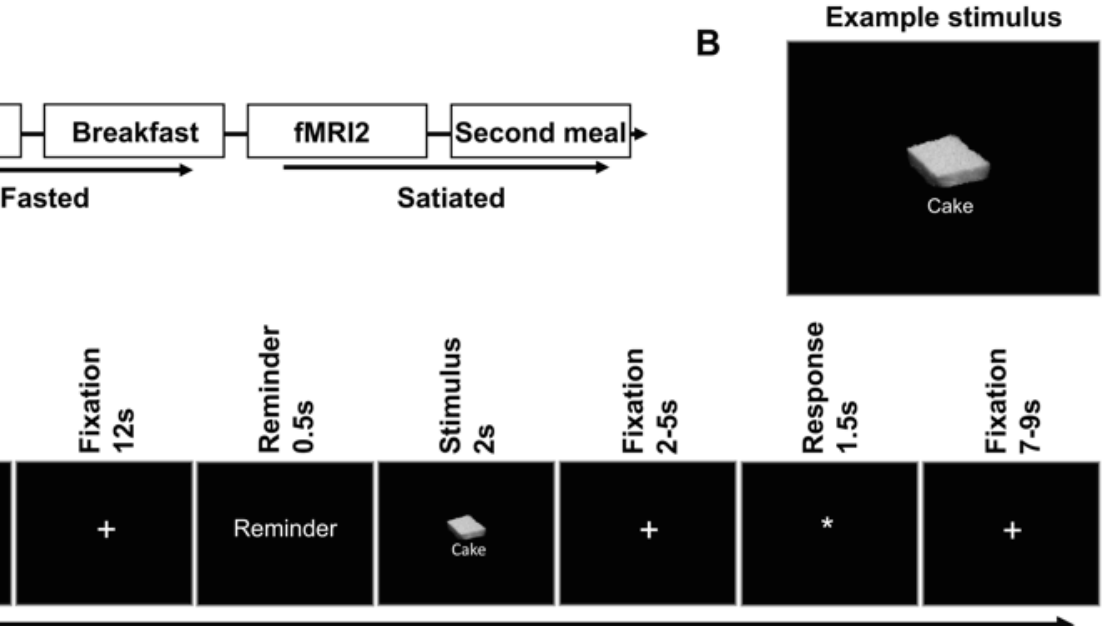

Example stimulus

$$
\begin{aligned}
& 4 x \text { Liking } \\
& 4 x \text { Wanting }
\end{aligned}
$$
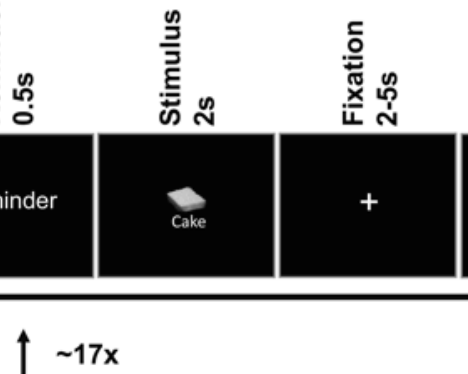

号
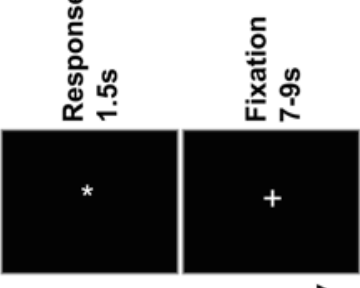

Figure 1:

(A) Test day schematic overview. Subjects arrived fasted between 8 and 9 am. Each fMRI session took approximately 45 minutes including a functional MRI run of 35-minute MRI. Subjects were given 30 minutes to consume their meal after each scan. (B) Layout of the stimuli slides: A centered food image that was standardized for size color with the food name below the image. (C) Overview of the stimulus presentation during the fMRI run. Stimuli were shown in 4 segments (dotted line) containing $\sim 17$ event related stimulus trials (solid line). Segments were presented in counterbalanced order. 


\section{Table 1:}

Correlation coefficients for the relationship between wanting task related signaling in the specified regions and BMI with a linear and quadratic model, and related to the average wanting rating for the trials included in the analysis.

\begin{tabular}{|c|c|c|c|c|}
\hline & & BMI & & $\begin{array}{c}\text { Average wanting } \\
\text { rating }\end{array}$ \\
\hline Wanting TRS in the & $\begin{array}{l}\text { Linear } \\
\text { r value }\end{array}$ & r value & ${ }^{i c}$ Nadir BMI & $r$ value \\
\hline $\begin{array}{r}\text { Caudate } \\
\mathbf{L} \\
\mathbf{R}\end{array}$ & $\begin{array}{l}0.134 \\
0.179\end{array}$ & $\begin{array}{l}0.354 \\
0.287\end{array}$ & $\begin{array}{l}24.36 \\
24.51\end{array}$ & $\begin{array}{l}0.118 \\
0.093\end{array}$ \\
\hline $\begin{array}{r}\text { Hypothalamus } \\
\mathbf{L} \\
\mathbf{R} \\
\text { Putamen }\end{array}$ & $\begin{array}{l}0.192 \\
0.191\end{array}$ & $\begin{array}{l}0.531 \# \\
0.514 \#\end{array}$ & $\begin{array}{l}25.25 \\
24.76\end{array}$ & $\begin{array}{l}0.344 \\
0.298\end{array}$ \\
\hline $\mathbf{L}$ & $\begin{array}{c}0.377 \# \\
0.457\end{array}$ & $\begin{array}{l}0.469 \# \\
0.500 \#\end{array}$ & $\begin{array}{l}22.88 \\
21.03\end{array}$ & $\begin{array}{l}0.176 \\
0.260\end{array}$ \\
\hline Overall: & $0.255^{*}+$ & $0.321^{\star \star *}$ & 23.80 & $0.215^{\star \star}$ \\
\hline
\end{tabular}

\# Correlation significant at $P<0.05$, ${ }^{*}$ versus zero $P<0.05$, corrected, ${ }^{* *}$ versus zero $P<0.01$, corrected,
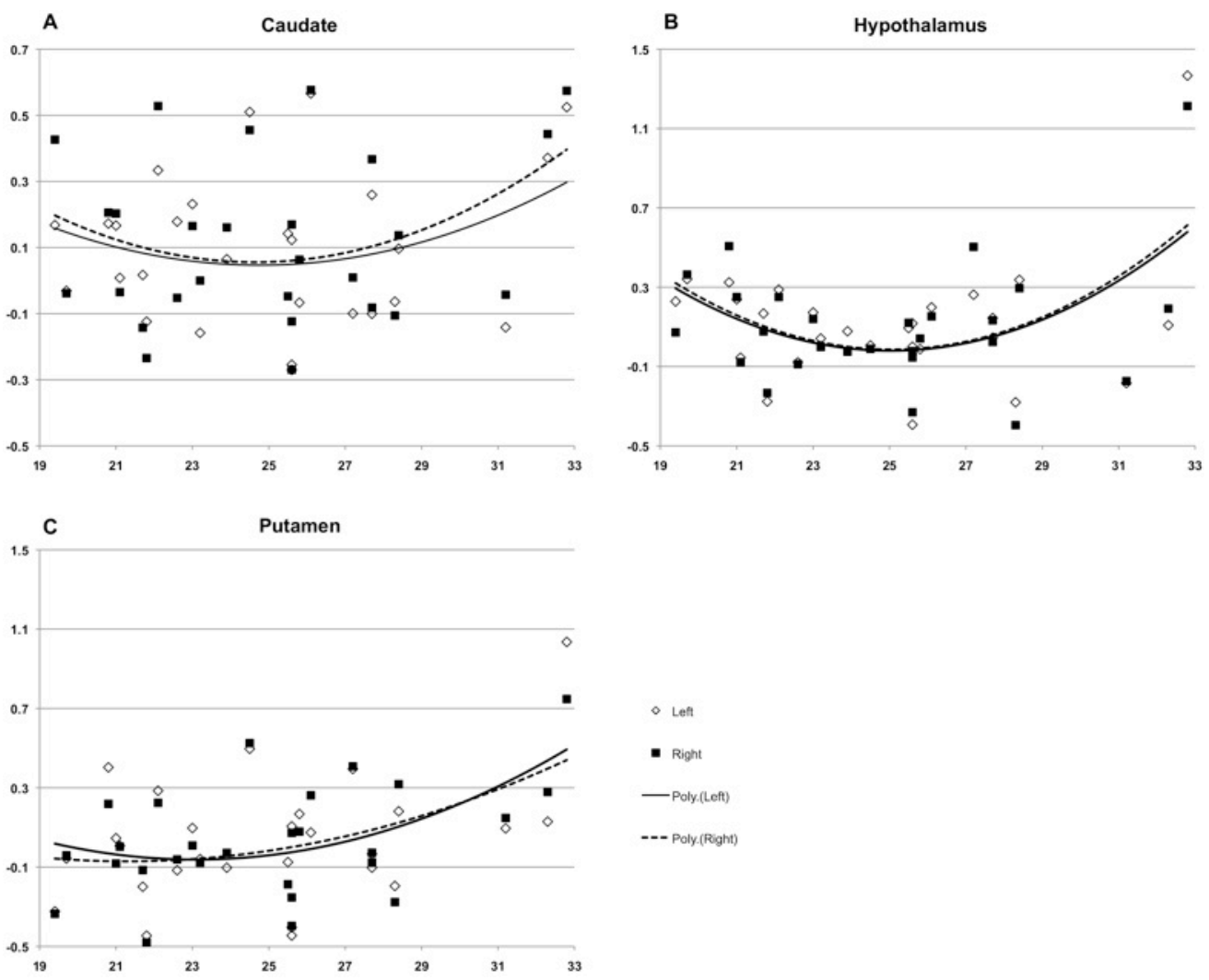

Figure 2:

The quadratic model for the relation between post-meal wanting task related signaling and BMI. (A) In the caudate left: $y=0.0039 x^{2}-0.19 x+2.42, R^{2}=0.082$; right $y=0.0051 x^{2}-0.25 x+3.18$, $R^{2}=0.126$. (B) In the hypothalamic region left: $y=0.0099 x^{2}-0.50 x+6.20, R^{2}=0.265$; right $y=0.0105 x^{2}-0.52 x+6.55, R^{2}=0.282$. (C) In the Putamen left: $y=0.0059 x^{2}-0.27 x+3.08, R^{2}=0.220$; right $y=0.0039 x^{2}-0.164 x+1.67, R^{2}=0.250$. 
of food and food intake, behavioral liking and wanting were analyzed using the ratings given in the scanner. Here, the question to determine liking was "how much do you like this item, not considering if you want to eat it right now?". Wanting was determined using the question "how much do you want to eat this item right now?", while the subjects were instructed that the items that were rated high in wanting were to be consumed immediately after the scan. Behavioral liking was thereby defined as explicit food preference and behavioral wanting was defined as explicit food choice.

\section{Data analysis}

To analyze the brain activation, separate predictors for liking and wanting were created, to be used in general linear model analyses and subsequent second level analyses: Subjects rated the images for all items, thus each image was ranked according to the average rating given for the respective item. From this ranking, the five highest and five lowest images were selected to avoid an unbalanced design. Consequently, the predictors obtained were 'low liking', 'liking', 'low wanting' and 'wanting'. The duration of each trial was defined as the duration of the image presentation and the trials were modeled using the standard canonical two-gamma hemodynamic response function. Furthermore, predictors containing the nonselected trials were made and a linear habituation predictor was added using the BVA-Predictor tool (BVA-Predictor tool, J.M. Born, Maastricht). In the second study, additionally, onsets of instructions, reminder and response request were modeled. To perform group contrasts comparing the pre-meal and post-meal conditions, dummy coding was applied using the BVA-Predictor tool. Group effects were determined by second level random effects analysis (ANOVA) using all GLM factors. Neural correlates of wanting were related to BMI in hungry as well as satiated conditions. From the regions of interest, parameter estimates for the high wanting models were extracted and corrected for baseline, in which baseline represents the parameter estimates for the constant in the GLM/ANOVA model. All data were analyzed using Microsoft Excel 2008 for Macintosh and PASW statistics 18.

Simple Pearson's $r$ correlations were made between wanting task related signaling in the specified regions and BMI. In addition second order polynomial model was applied using Microsoft Excel and significances were assessed using PASW statistics (Figure 2 and Table 1). Consistency of correlation results between wanting task related signaling with BMI for both linear and quadratic models, was estimated by testing the averaged correlation coefficients from significant regions (all included regions) against zero, using a student's t-test. Finally, the correlation between the average wanting rating and the wanting task related signal was assessed using using Pearson's $r$ correlation and the effect of including this wanting rating in the linear/nonlinear model was assessed using multiple regression analyses. 


\section{Results}

\section{Hunger and satiety}

The hungry versus satiated condition was confirmed by high ratings for hunger premeal $(62.2 \pm 3.5 \mathrm{mmVAS})$ and low ratings post-meal $(10.8 \pm 1.7$, difference $p<0.001)$. The decrease in hunger over breakfast was statistically significant $(p<0.001)$.

\section{BMI related to behavioral measures}

BMI was positively related to hunger post-meal $(R=0.48, P=0.01)$, while it was not related to energy intake and behavioral liking and wanting as well as liking and wanting TRS in any region. In addition, in no region was a relationship found between a combined model of body weight and hunger and wanting TRS post-meal using a multiple regression analysis.

\section{$B M I$ related to wanting TRS}

To assess the way in which BMI relates to wanting task related signaling (TRS), we compared a quadratic model linear model of BMI when related to wanting TRS. Premeal, no correlations between wanting TRS and BMI was found. Conversely, postmeal, several significant results were seen: Over all three regions, the correlation coefficient was higher using a quadratic model $(P<0.02)$, showing the lowest wanting TRS with a BMI of 24. Individually, four significant quadratic correlations were found (bilaterally in the putamen and hypothalamus) compared with only one significant result (left putamen) when using a linear model (Table 1). The relationship between nonlinear BMI and wanting task related signaling was most pronounced in the hypothalamic region (Table 1 and Figure 1). These findings show that the relationship between wanting related signaling and $\mathrm{BMI}$ is highly consistent when using a nonlinear model, indicating that after eating a meal, BMI predicts wanting task related signaling such that signaling is lowest around BMI 24 (Table 1). In both, subjects with a BMI lower or higher than this value, wanting task related signaling was progressively higher, dependent on the distance from this BMI value.

\section{Discussion}

Here we present data that directly supports a nonlinear relationship between BMI and wanting task related signaling, especially in the hypothalamic region, but also in the striatum. This may explain some of the problems in describing the way in which BMI affects brain signaling: Previously, BMI was related with reward-related signaling in the striatum and with specifically wanting task related signaling in the hypothalamic region 9,14,19. However, the outcomes of different studies published appeared contradicting, in part reporting that overweight and obesity leads to increased brain signaling in reward-related and taste-related brain regions ${ }^{20,21}$ while others support the idea of reduced brain signaling in reward-related brain regions, indicating reward deficiency in subjects with a higher $\mathrm{BM} \mathrm{I}^{9,14,16,21}$. We show that a nonlinear model nadir at a BMI between 22 and 25 is the best model for the relation between $\mathrm{BMI}$ and signaling in the hypothalamic region and putamen, and to a lesser degree for the relation between $\mathrm{BMI}$ and signaling in the caudate. In the lower range of BMI 19-24 the model implies that wanting task related signaling in several regions is lower with higher BMI. Our own previous studies showed similar 
relationships between wanting TRS and BMI in subjects with similar weight range. Conversely, the new data regarding a BMI of 24-33 implies a positive relationship between wanting TRS and BMI. No relationships were found between hunger and wanting TRS, or for a combined model of hunger and BMI, confirming that differences in hunger did not account for the effects on wanting TRS that were observed. Taken together, the data suggest that sensitivity rather than deficiency in signaling of the rewarding value of food may drive excessive energy intake in overweight and obese subjects.

Previouws studies showed that genetic differences lead to different directionality in the correlations, offering more possible explanations for the seemingly contradictory effects $^{19,22}$ : In these studies, subjects with an obesity prone genetic background exhibited a positive correlation between BMI and brain activation and subjects without this obesity susceptibility exhibited a negative correlation. Depending on the distribution of genotypes, this may also be modeled with a nonlinear model. The lack of a significant relationship between BMI and wanting TRS in the nucleus accumbens, is in line with our earlier study, in which we did not see an involvement of this region predominantly with liking in relation to cognitive dietary restraint ${ }^{16}$.

Furthermore, previous studies have shown that the drive to eat was positively related to activation in multiple brain regions ${ }^{23}$. Thin subjects exhibited reduced signaling related to food images, while reduced obese showed relatively increased signaling when in a positive energy balance ${ }^{24}$. Taken together, this suggests that normal weight individuals, may be biased towards a BMI of approximately 24 by reward sensitivity: The lower the $\mathrm{BMI}$, the higher the brain activation in wanting related, homeostatic regions during food choice in the absence of hunger ${ }^{25}$. It is possible that this bias implies a drive towards the body weight that is associated with the most favorable survival rate and overall health, i.e. a BMI of approximately 24-2526,27. Conversely, in subjects with a BMI of 25-33, we found that wanting task related signaling was higher in subjects with a higher BMI. Thus these overweight and obese subjects may experience motivation for an even higher energy intake in the absence of hunger, biasing them towards an excessive energy intake resulting in maintenance of overweight or even positive energy balance resulting in unfavorable weight gain ${ }^{23}$. The results from this study agree with earlier findings for subjects with a $\mathrm{BMI}<25$, in which overall brain signaling, including signaling in the hypothalamic region, was inversely related with $\mathrm{BMI}^{9,16}$. In addition, the present study is in line with recent findings that indicated a positive relationship between reward or gustatory signaling, with overweight and obesity, thereby implying a body weight related susceptibility for overweight and obesity $20,22,28$. Obviously, brain signaling processes associated with food choice, food viewing or food tasting can only explain a part of body weight regulation: Previous studies have shown that dietary restraint, disinhibition and feelings of hunger also affect food intake 29,30 . Thereby, the susceptibility to weight change may thus be modulated or even overruled by complex cognitive processes, preventing some subjects from gaining body weight. However, the limited success rate of weight maintenance after successful weight loss, which is reportedly only about $20 \%$ of the subjects that lost significant amount of weight ${ }^{31,32}$, may be due to an underlying susceptibility in brain signaling, which may ultimately also originate from the genetic background ${ }^{19,22,33}$.

In conclusion, the relationship between reward-related brain signaling and BMI was better characterized by a nonlinear model compared with a linear model, especially 
in the hypothalamic region and putamen. This nonlinearity may explain contradicting past findings and may imply the lowest motivation to eat at a BMI of approximately 25 , stimulating weight gain in thin individuals, and supporting overweight and obesity in the overweight and obese.

\section{References:}

1 Seidell, J. C. Epidemiology and health economics of obesity. Medicine 34, 506-509 (2006).

2 Catenacci, V. A., Hill, J. O. \& Wyatt, H. R. The Obesity Epidemic. Clinics in Chest Medicine 30, $415-444$ (2009).

3 Westerterp, K. R. \& Speakman, J. R. Physical activity energy expenditure has not declined since the 1980s and matches energy expenditures of wild mammals. Int J Obes 32, 1256-1263 (2008).

4 Rutters, F., Nieuwenhuizen, A. G., Lemmens, S. G. T., Born, J. M. \& Westerterp-Plantenga, M. S. Acute Stressrelated Changes in Eating in the Absence of Hunger. Obesity 17, 72-72 (2009).

5 Epstein, L. H., Leddy, J. J., Temple, J. L. \& Faith, M. S. Food reinforcement and eating: a multilevel analysis. Psychological bulletin 133, 884-906 (2007).

6 Davis, C. et al. From motivation to behaviour: a model of reward sensitivity, overeating, and food preferences in the risk profile for obesity. Appetite 48, 12-19 (2007).

7 Franken, I. H. \& Muris, P. Individual differences in reward sensitivity are related to food craving and relative body weight in healthy women. Appetite 45, 198-201, doi:S0195-6663(05)00042-5 [pii] 10.1016/j.appet.2005.04.004 (2005)

8 Wang, G. J. et al. Brain dopamine and obesity. Lancet 357, 354-357 (2001).

9 Born, J. M. et al. Acute stress and food-related reward activation in the brain during food choice during eating in the absence of hunger. Int J Obes 34, 172-181 (2010).

10 Blum, K. et al. Reward deficiency syndrome: a biogenetic model for the diagnosis and treatment of impulsive, addictive, and compulsive behaviors. J Psychoactive Drugs 32 Suppl, i-iv, 1-112 (2000).

11 Kenny, P. J. Brain reward systems and compulsive drug use. Trends Pharmacol Sci 28, 135-141, doi:S0165-6147(07)00027-2 [pii] 10.1016/j.tips.2007.01.008 (2007).

12 Koob, G. F. \& Volkow, N. D. Neurocircuitry of Addiction. Neuropsychopharmacology 35, 217-238 (2009).

13 Allison, D. B. et al. Antipsychotic-Induced Weight Gain: A Comprehensive Research Synthesis. Am J Psychiatry 156, 1686-1696 (1999).

14 Davis, C. \& Fox, J. Sensitivity to reward and body mass index (BMI): evidence for a non-linear relationship. Appetite 50, 43-49 (2008).

15 Berridge, K. C. Food reward: brain substrates of wanting and liking. Neurosci Biobehav Rev 20, 1-25 (1996).

16 Born, J. M., Lemmens, S. G. T., Formisano, E., Goebel, R. \& Westerterp-Plantenga, M. S. Differences between liking and wanting signals in the human brain and relations with cognitive dietary restraint and body mass index. Am J Clin Nutr 94, 392-403, doi:doi:10.3945/ajcn.111.012161 (2011).

17 Goebel, R., Esposito, F. \& Formisano, E. Analysis of functional image analysis contest (FIAC) data with brainvoyager QX: From single-subject to cortically aligned group general linear model analysis and selforganizing group independent component analysis. Human Brain Mapping 27, 392-401, doi:10.1002/hbm.20249 (2006).

18 Knutson, B. \& Gibbs, S. Linking nucleus accumbens dopamine and blood oxygenation. Psychopharmacology 191, 813 (2007).

19 Stice, E., Spoor, S., Bohon, C. \& Small, D. M. Relation Between Obesity and Blunted Striatal Response to Food Is Moderated by TaqIAA1 Allele. Science 322, 449-452, doi:10.1126/science.1161550 (2008).

20 Stice, E., Yokum, S., Burger, K. S., Epstein, L. H. \& Small, D. M. Youth at Risk for Obesity Show Greater Activation of Striatal and Somatosensory Regions to Food. The Journal of Neuroscience 31, 4360-4366, doi: 10.1523/jneurosci.6604-10.2011 (2011).

21 Martin, L. E. \& Potts, G. F. Reward sensitivity in impulsivity. Neuroreport 15, 1519-1522, doi: 00001756-200406280-00032 [pii] (2004).

22 Stice, E., Yokum, S., Bohon, C., Marti, N. \& Smolen, A. Reward circuitry responsivity to food predicts future increases in body mass: Moderating effects of DRD2 and DRD4. Neuroimage 50, 1618-1625 (2010).

23 Beaver, J. D. et al. Individual Differences in Reward Drive Predict Neural Responses to Images of Food. J. Neurosci. 26, 5160-5166, doi:10.1523/jneurosci.0350-06.2006 (2006).

24 Cornier, M.-A. et al. The Effects of Overfeeding on the Neuronal Response to Visual Food Cues in Thin and Reduced-Obese Individuals. PLoS ONE 4, e6310 (2009).

25 Berthoud, H.-R. Multiple neural systems controlling food intake and body weight. Neuroscience \& Biobehavioral Reviews 26, 393-428 (2002).

26 Allison, D. B., Faith, M. S., Heo, M. \& Kotler, D. P. Hypothesis Concerning the U-shaped Relation between Body Mass Index and Mortality. American Journal of Epidemiology 146, 339-349 (1997).

27 Orpana, H. M. et al. BMI and Mortality: Results From a National Longitudinal Study of Canadian Adults. Obesity $18,214-218$ (2009). 
28 Gearhardt, A. N. et al. Neural Correlates of Food Addiction. Arch Gen Psychiatry, archgenpsychiatry.2011.2032, doi:10.1001/archgenpsychiatry.2011.32 (2011).

29 Pasman, W. J., Saris, W. H. \& Westerterp-Plantenga, M. S. Predictors of weight maintenance. Obes Res 7, 43-50 (1999).

30 Vogels, N. et al. Relation of weight maintenance and dietary restraint to peroxisome proliferator-activated receptor gamma2, glucocorticoid receptor, and ciliary neurotrophic factor polymorphisms. The American Journal of Clinical Nutrition 82, 740-746 (2005).

31 Wing, R. R. \& Phelan, S. Long-term weight loss maintenance. The American Journal of Clinical Nutrition 82, 222S-225S (2005).

32 Anderson, J. W., Konz, E. C., Frederich, R. C. \& Wood, C. L. Long-term weight-loss maintenance: a metaanalysis of US studies. The American Journal of Clinical Nutrition 74, 579-584 (2001).

33 DelParigi, A. et al. Persistence of abnormal neural responses to a meal in postobese individuals. Int $\mathrm{J}$ Obes Relat Metab Disord 28, 370-377 (2003). 
Chapter 4

\section{Acute stress and food-related reward activation in the brain during food choice during eating in the absence of hunger}

Jurriaan M Born, Sofie GT Lemmens, Femke Rutters, Arie G Nieuwenhuizen, Elia

Formisano, Rainer Goebel, and Margriet S Westerterp-Plantenga, Int J Obes 2010;34:172-181. 


\section{Abstract}

Background: Stress results in eating in the absence of hunger, possibly related to food reward perception.

Hypothesis: Stress decreases food reward perception.

Aim: Determine the effect of acute stress on food choice and food choice reward related brain activity.

Subjects: 9 females (BMl=21.5 $\pm 2.2 \mathrm{~kg} / \mathrm{m} 2$, Age $=24.3 \pm 3.5 \mathrm{y})$.

Procedure: Fasted subjects came twice to randomly complete either a rest or stress condition. Per session, two functional MRI scans were made, wherein the subjects chose the subsequent meal (food images). The rewarding value of the food was measured as liking and wanting. Food characteristics (e.g. crispiness, fullness of taste etc.), energy intake, amount of each macronutrient chosen, plasma cortisol and VAS hunger and satiety were measured.

Results: Fasted state was confirmed by high hunger $(80 \pm 5 \mathrm{mmVAS})$. Breakfast energy intake $(3 \pm 1 \mathrm{MJ})$ and liking were similar in all conditions. Wanting was lower postprandially $(\Delta=-.3$ items/category, $p<.01)$. Breakfast decreased hunger $(-42$ mmVAS, $p<.01)$. Postprandially, energy intake $(-1.1 \mathrm{MJ})$, protein intake $(-14.7 \mathrm{~g})$ and carbohydrate intake $(-32.7 \mathrm{~g}$ all $\mathrm{p}<.05)$ were lower. Fat intake was not different $(-7.3$, $\mathrm{p}=.4$ ). Putamen activity was not lower postprandially. Cortisol levels were increased in the stress condition (cortisol $\triangle \mathrm{AUC}=+2.2 \cdot 104 \mathrm{nmol} \cdot \mathrm{min} / \mathrm{l}, \mathrm{p}<.05$ ). Satiety was lower after breakfast $(-8 \mathrm{mmVAS}, p<0.01)$. Postprandial energy intake, protein intake and carbohydrate intake was relatively higher compared with the rest condition, resulting from more choice for crispiness and fullness of taste $(p<.05)$. Brain activation was reduced in reward-areas: Amygdala, hippocampus and cingulate cortex (AUC=-13.33, -1.34, -2.56 \%BOLD.sec for choosing breakfast and AUC=-9.31, $-1.25,-2.34 \%$ BOLD $\sec <.05$ for choosing the second meal). Putamen activation was decreased postprandially (AUC $=-1.2 \%$ BOLD $\cdot$ sec, $p<.05$ ).

Conclusion: Reward signaling and reward sensitivity was significantly lower under stress, coinciding with increased energy intake from food choice for more crispiness and fullness of taste. The changes in putamen activation may reflect specifically decreased reward prediction sensitivity. 


\section{Introduction}

The currently rising obesity epidemic in the developed world is drawing increasing attention $^{1}$, especially since visceral (or central) obesity is a key factor in the development of the metabolic syndrome ${ }^{2,3}$. It has been shown that increased stress indices coincide with the increased prevalence of obesity and the metabolic syndrome $e^{4,5}$. Stress is indicated by increased activity of the hypothalamus pituitary adrenal axis (HPA axis) and is represented by elevated plasma cortisol levels ${ }^{6,7}$. It has been shown that viscerally obese subjects often have increased cortisol levels compared with normal weight subjects ${ }^{8}$. Furthermore, an interaction between cortisol and leptin has been shown: the obesogenic effects of leptin deficiency in rats can be reversed by subsequent adrenalectomy ${ }^{9}$. This indicates that both leptin and cortisol signaling simultaneously contribute to food intake regulation. Other studies have indicated that also insulin levels are positively related to cortisol levels and that insulin and cortisol have antagonistic effects on each other in terms of feeding behavior and body weight ${ }^{10,11,12}$. Furthermore, it was shown that cortisol and insulin co-determine the macronutrient intake in rats, such that higher insulin levels were associated with higher lard intake in rats ${ }^{13}$.

Additionally, other studies suggest that high cortisol levels leads to altered food choice ${ }^{14,15,16}$ and stress is thought to result in food choice for items with a higher content of fat and sweet, which are perceived as highly rewarding ${ }^{15,17,18,19,20}$. Over all, this is striking evidence that stress influences food choice through various hormonal pathways.

Eating behavior that may be influenced by stress is divided into two components: The first component is homeostatic eating, which relates to hunger and satiety, and ultimately to energy balance and steady body weight. The second component is non-homeostatic eating, which is influenced by food reward, and is observed as eating in the absence of hunger ${ }^{21}$. Westerterp and Speakman (2008) performed an extensive meta analyses on energy expenditure data of cohorts around the world. They showed that the energy expenditure of adults was related to factors such as BMI, but did not decrease at all between 1988 and $2006^{22}$. This suggests that the recent rise in obesity may result from non-homeostatic eating over long periods of time, rather than a lack of physical exercise. Furthermore, we showed that stress leads to eating in the absence of hunger in subjects with high disinhibition scores ${ }^{23}$. This suggests that stress interferes with non-homeostatic pathways that are involved in food intake regulation, such as food reward signaling, resulting in a shift of food choice towards foods with high fat and carbohydrate content ${ }^{23}$. Taken together, we hypothesize that stress significantly decreases the food reward, leading to nonhomeostatic eating in the absence of hunger, with food choices that result in higher energy intakes.

The rewarding value of food can be described as consisting of two components: liking, which is the hedonic preference for a given food item and wanting, which is the motivation to obtain the food item ${ }^{24}$. The combination of liking and wanting, defines the rewarding value of a given item and thereby its specific maximum perceived food reward. At the level of the central nervous system, it has been shown that reward activates dopamine mediated signaling in key areas of the brain. In studies that investigated food reward specifically with various paradigms, using food images, smells and tastes, brain areas involved were the amygdala, striatum, 
hippocampus anterior cingulate cortex and orbitofrontal cortex ${ }^{25,26,27,28,29,30}$. Previous studies have shown that dopamine release in these brain areas is dependent on satiety, but also on body mass index ${ }^{31,32}$, which by itself has been inversely correlated with dopamine mediated signaling ${ }^{32}$. This indicates that dopamine reward signaling may be involved in food intake regulation. Finally, several reports show interactions between dopamine reward systems and cortisol, or corticotropin releasing factor $33,34,35,36,37$, thus suggesting that a state of endocrinological stress may directly lead to dramatic changes in food reward signaling.

We hypothesize that acute stress reduces signaling in food reward circuits, thereby leading to eating in the absence of hunger. To test the hypothesis that stress reduces the rewarding effect of food in general, we aimed to determine the effects of acute stress on food reward related brain activation that was induced by food choice.

\section{Methods}

\section{Subjects}

The study was approved by the Medical Ethical Committee of the University Maastricht, and informed, written consent was obtained from all subjects. Inclusion criteria were: female, normal body weight (BMI 19-25), right-handedness. Exclusion criteria were recent dieting, a personal or familial history of psychiatric disorder, or intra uterine contraceptives. Fourteen test subjects were screened and ten of them were included with the following characteristics: Age $=24.1 \pm 1.1, \mathrm{BMI}=21.5 \pm 0.7$, Three factor eating questionnaire $\mathrm{TFEQ}^{38}$ dietary restraint $=8.4 \pm 1.1$, disinhibition $=$ $4.2 \pm 0.5$, emotional eating $=4.6 \pm 0.9$, age $=24.3 \pm 1.2$, State trait anxiety inventory, STAI-2 (trait) $=37.8 \pm 6.2$.

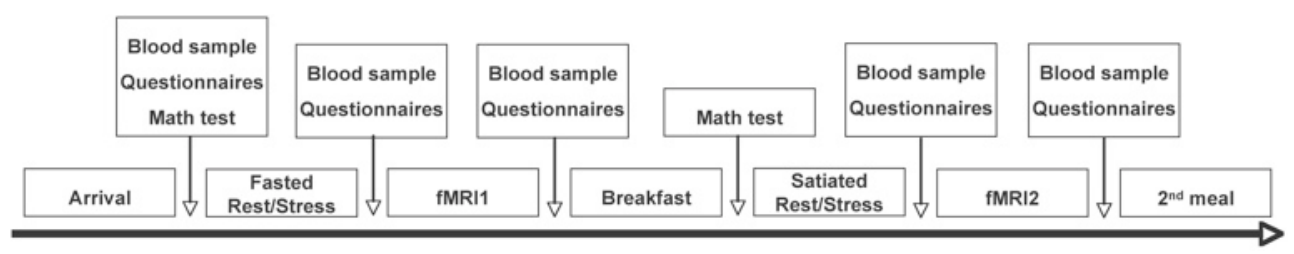

Figure 1: Schedule of the activities that subjects conducted per test session.

\section{Sessions}

Subjects came to the university twice in the fasted state. Randomly two sessions were completed, one in the rest condition and one in the stress condition, each at least one week apart. To create stress versus rest, an unsolvable versus solvable mathematical test was given before each scan. This test has been described and validated before ${ }^{23,39}$. A schedule of how the sessions were executed is given in Figure 1. Each session included five questionnaires and five blood samples, two fMRI scans and two meals (breakfast after the first scan and a postprandial meal after the second scan) that were chosen from food items shown within the scanner (Table 1). The meals were offered immediately after the questionnaires and blood samples were obtained quickly after the scan. 


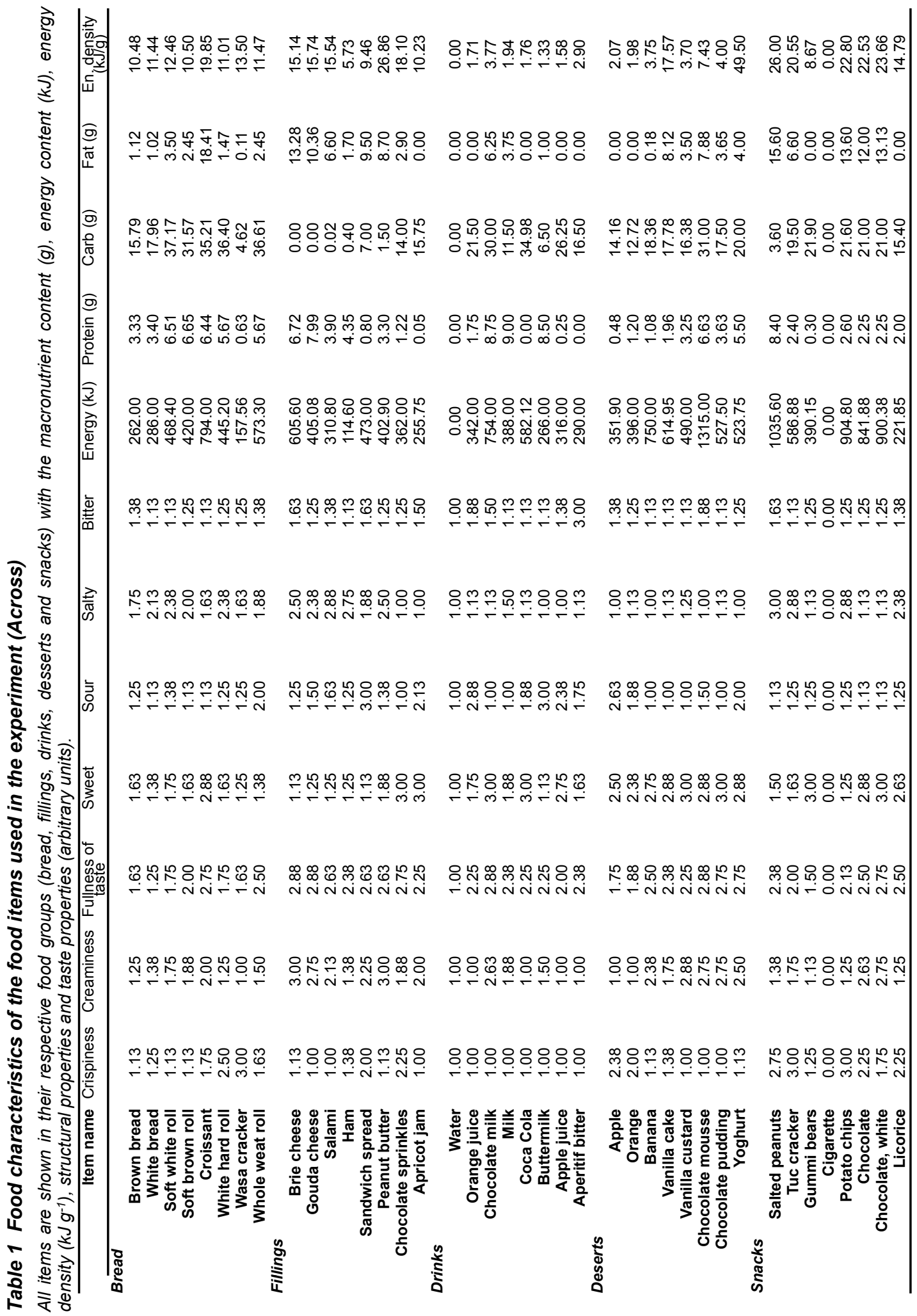




\section{Visual analog scales (VAS)}

To determine the effect of the breakfast and a postprandial meal, visual analog scales for hunger and satiety were taken five times: baseline $>20$ min after placing the cannula, before the first scan, immediately after the first scan before breakfast, before the second scan and immediately after the second scan before the second meal. The VAS questionnaires consisted of $100 \mathrm{~mm}$ lines, anchored with "not at all" at the far left "extremely" at the far right. Questions asked were "How hungry are you?", "How full do you feel?", "How satiated do you feel?", "How thirsty are you?", and "How large is your desire to eat?"

\section{Hormones}

At the beginning of the test session, a cannula was placed in the antecubital vein, which was kept there for the duration of the session. After each time that the questionnaires were filled out, a blood sample for hormone measurements was drawn $(10 \mathrm{~mL})$. Thus in total five blood samples were obtained starting $>20 \mathrm{~min}$ after placing the cannula. These samples were each divided over two tubes: $6 \mathrm{~mL}$ was transferred into a serum tube (BD Vacutainer with separation gel, $8.5 \mathrm{~mL}$ ). Blood serum was obtained by centrifugation $\left(4^{\circ} \mathrm{C}, 3000 \mathrm{RPM}, 10 \mathrm{~min}\right)$. The remaining 4 $\mathrm{mL}$ were transferred into a plasma tube (BD Vacutainer with EDTA, $10 \mathrm{~mL}$ ). Blood plasma was obtained by centrifugation $\left(4^{\circ} \mathrm{C}, 3000 \mathrm{RPM}, 20 \mathrm{~min}\right)$. All samples were frozen and stored at $-80^{\circ} \mathrm{C}$ until further analysis. Serum cortisol levels for stress were determined by radio immuno assay (at Département des Sciences Fonctionnelles, B41 - Physiologie de Reproduction, Liège, Belgium). Both leptin and insulin were determined in our own lab from the plasma samples, by means of radio immuno assay according to the manufacturer's instructions (Human insulin specific and human leptin specific RIA kit, Linco Research, St. Charles, Missouri, USA).

\section{Rewarding value}

Rewarding value was determined as liking and wanting. To obtain data on the rewarding value of food and food intake, liking and wanting were analyzed as follows: the food items used in this study were ranked based on their relative liking in a separate set of experiments 40 . In our study, the liking scores refer to the average of the predetermined liking scores of all the chosen items for one meal. To determine these scores, all food items were shown to the same subjects in random pairs. Each food item was shown paired with all other items within the category and subsequently the items were additionally paired between the categories. By selecting the preferred items from each pair, a relative ranking was obtained for all items ${ }^{40}$.

To determine which food was wanted by our subjects, food items were chosen inside the scanner. These items had to be eaten immediately after the scan. The choice for the respective item together with the average number of items chosen therefore reflects wanting.

\section{Food characteristics}

To determine the characteristics of the selected food, the structural characteristics of each food item were determined by a separate ten-subject taste panel. All food items were offered individually and in randomized order. Water was provided to 


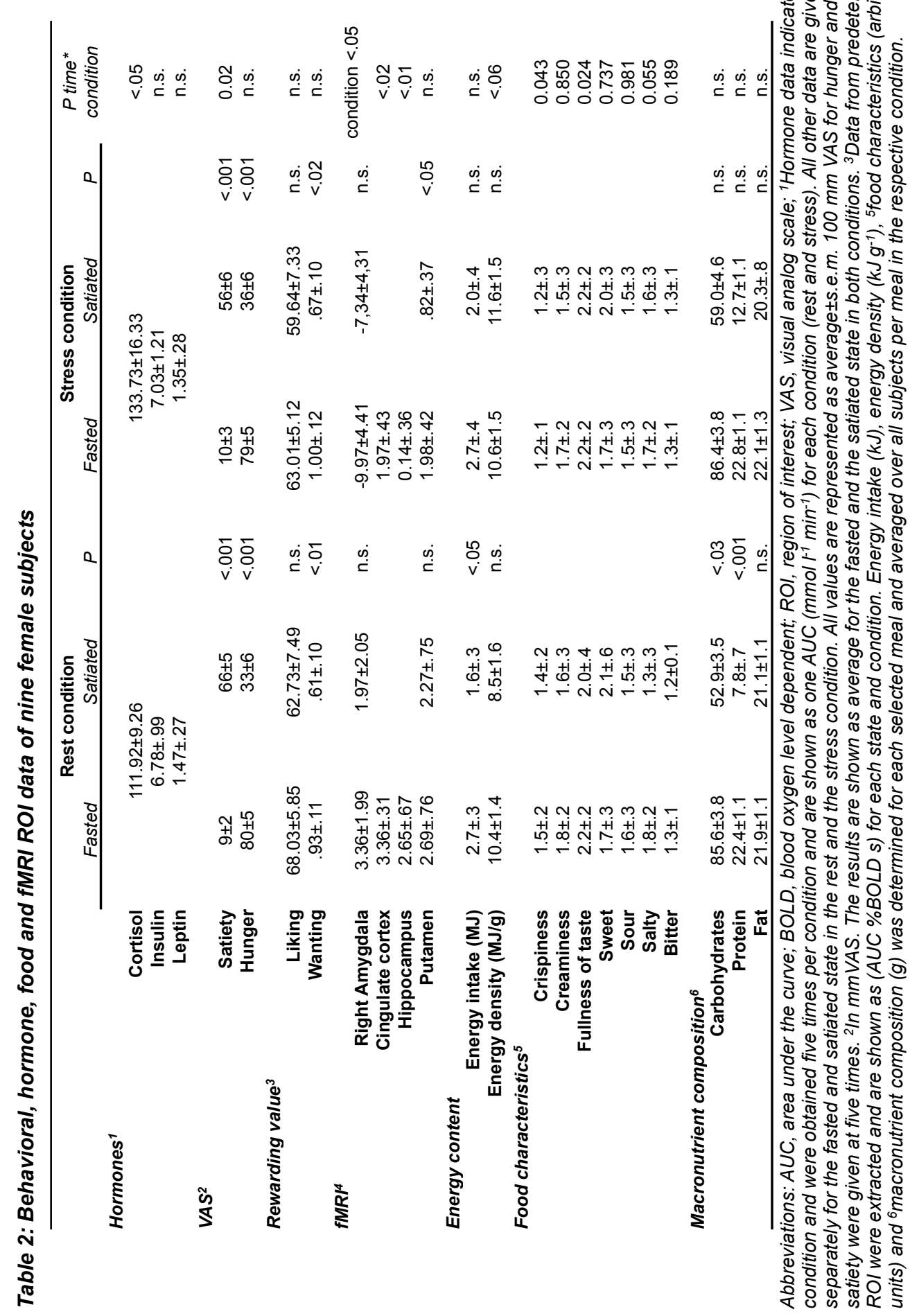


avoid cross tasting. The taste dimensions that were tested were crispiness, creaminess, fullness of taste, sweet, sour, salty and bitter. Fullness of taste was defined by instructing the test panel to consider how strong the taste of a given food item was as well as how much this taste filled the mouth. All dimensions were rated on a seven-point scale, ranging from "not at all" to "extremely" for each food item and each dimension. The average ratings were calculated and used in the subsequent analyses. Furthermore, macronutrient composition, total energy content and the average energy density of the chosen food were calculated (Table 1).

\section{Functional MRI}

The subjects were placed in supine position in a Siemens Magnetom Allegra, with the standard one-channel head coil. The subjects position was confirmed with T1 weighted scout images. In the functional MRI scanner, the subjects were successively presented with five categories of food items: bread, fillings, drinks, desserts and snacks (Table 1). The subjects knew all items from a similar experiment outside the scanner, as well as the quantity that they were offered. For each category, a menu of eight images of the available food items was shown for ten seconds. The subjects were asked to choose between zero and two items from each of these menus to create their own meals, and they were instructed that those items had to be eaten completely immediately after the scan. To select the desired items, an MRI compatible joystick was used, which was fixed to the scan table at the waist at an angle that allowed comfortable usage. It was confirmed that the joystick was easily reached with the right hand to move the mouse pointer, and with the left hand to press a button to select the item. Before starting the food choices, subject had been in the MRI for 15 minutes, performing another behavioral paradigm. The food selection was preceded with 16 second gap (fixation cross), followed by written instruction regarding the subsequent food choice, which was followed by another 16 second gap. Food choice displays were separated by 16 second gaps. Functional MRI images were acquired throughout the session using a standard T2* weighted protocol to obtain blood oxygen level dependent (BOLD) T2* signal $(\mathrm{TR}=2 \mathrm{~s}, \mathrm{TE}=$ $25 \mathrm{~ms}$, Flip angle $=90^{\circ}$, matrix $=64 \times 64$, voxel size $3.5 \times 3.5 \times 3.5 \mathrm{~mm}$ ).

\section{Data analysis}

Hormone level measurements and behavioral data were analyzed using factorial ANOVA with or without repeated measures, depending on the variables that were included. Pearson correlations were made per condition. With the exception of the fMRI image data, all data were analyzed using Microsoft Excel and SPSS 16. For cortisol data, areas under the curve were calculated using the trapezoid method.

The fMRI data were imported into BrainVoyager QX (Brain Innovation B.V., Maastricht, The Netherlands). To preprocess the functional data, slice scan time correction with cubic spline interpolation, motion correction with trilinear interpolated motion estimation and subsequent sinc interpolation, and temporal high pass filtering with a window of 5 cycles was applied. One out of the ten subjects scanned, had to be excluded from analysis due to excessive movement during the scan.

The functional data were aligned to each subjects' own $1 \mathrm{~mm}$ isovoxel high resolution T1 weighted anatomical scan. First, the head tissues were digitally 
removed from the brain, to optimize the performance of BrainVoyager's auto alignment algorithm. Second the auto alignment was performed and corrected manually under visual inspection, if necessary. Finally, all images were transformed into the Talairach coordinate system 41 using the standard procedure in BrainVoyager. Statistical analyses were superimposed on a group average anatomical brain image.

To analyze the brain activation, predictors for general linear model (GLM) analysis were created: One predictor with a duration of 10 seconds, was created for each presentation of the menu from which the food had to be chosen. The predictors were modeled using the standard canonical two-gamma hemodynamic response function. Two predictor types were defined: menu presentation from which something was chosen, and menu presentation from which nothing was chosen. Group GLMs were performed, including the measurements for breakfast selection and the second meal selection (postprandially) in the rest and the stress condition. Group contrasts were used to compare activations in whole brain images and a functional voxel cluster threshold of $n=4 \times 27 \mathrm{~mm}^{3}$ was set.

Anatomical regions of interest (ROI) were determined from literature $25,26,27,28,29,30$ and generated bilaterally. The regions included the anterior cingulate cortex, amygdala, hippocampus, hypothalamus and putamen. Group contrasts for choosing something versus choosing nothing, revealed significantly higher activation in the right frontal cortex when something was chosen (Fig. 3A-C, FDR $p<0.05$ ). Additionally, it was found that signal in all ROls was higher on average in case that food items were chosen, therefore, the analyses of reward related activation were performed using the signal when food items were chosen. To determine functionally relevant $\mathrm{ROI}$ shapes, group contrasts were made for high wanting vs. low wanting, using the predetermined ROI definitions as a mask. Subsequently, the significantly active voxels from this contrast were exported as the new ROI definitions.

The average percent BOLD change was extracted using event related averaging. Events were defined as image presentations in which a choice was made for an item. The data that were extracted from the corrected ROI, was imported into Microsoft Excel. Subsequently, the data were normalized to time $t=0$ (the moment that the food menu was shown). The averages of all measurements were plotted per $\mathrm{ROI}$ and the presence of hemodynamic response curve in the BOLD signal was visually confirmed. The area under the curve from the image presentation start until 12 seconds ( $t=0$ to $t=12$ ) was calculated using the trapezoid method and further analyzed using SPSS 16 using ANOVA repeated measures. 


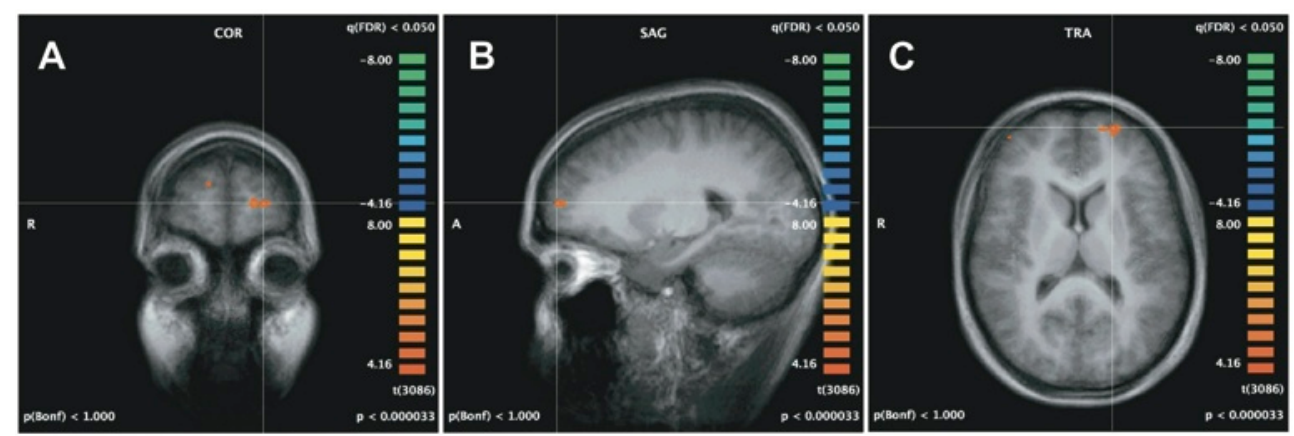

Figure 2:

Sagittal (a), coronal (b) and transversal (c) sections, showing the GLM contrast of choosing something versus choosing nothing. Significant activation is visible in the left frontal cortex $(-21,58,13 ; P<0.05$ FDR corrected).
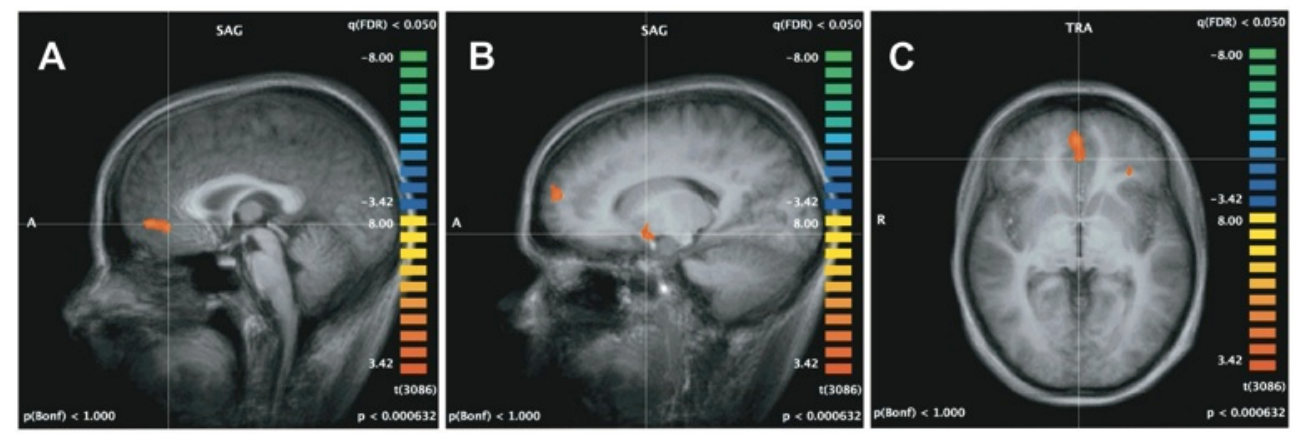

Figure 3:

Two sagittal $(a, b)$ sections at different planes and a transversal (c) section with the GLM contrast of choosing breakfast versus choosing a meal postprandially. There is a clear activation in the orbitofrontal cortex (3, 48, -1), frontal cortex $(13,61,20$ and -12, 63, 21) and putamen (18, 5, -5; P<0.05 FDR corrected).

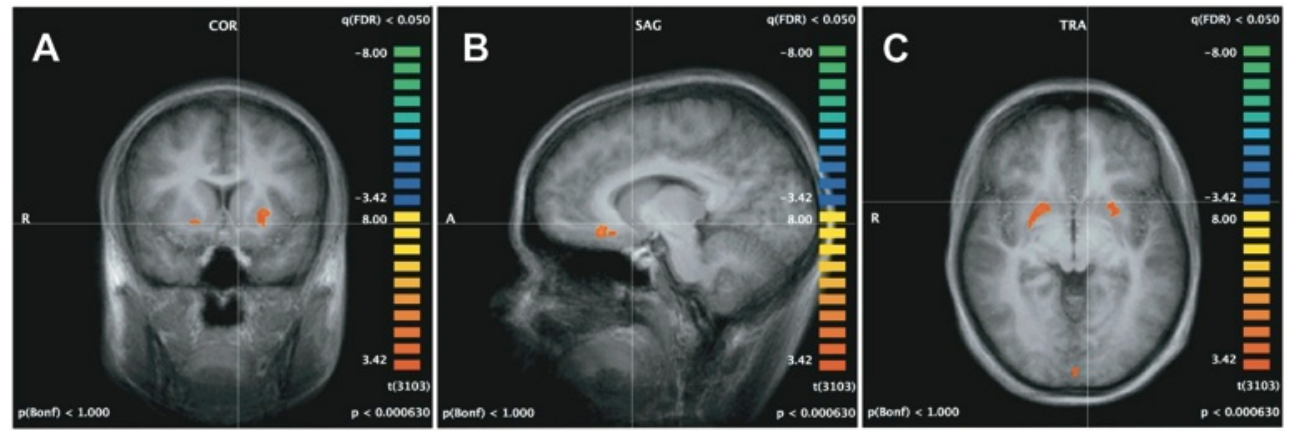

Figure 4:

Sagittal (a), coronal (b) and transversal (c) sections, showing the GLM contrast rest condition versus stress condition. Significant activation in present in the putamen (22, 4, -3 and $-23,11,4)$ and the orbitofrontal cortex $(-8,28,-6, P<0.05$ FDR corrected). 


\section{Results}

The fasted state before the first scan was confirmed by low VAS scores for satiety and fullness, and high scores for hunger, thirst and desire to eat. Eating the selfselected breakfast lead to significantly decreased hunger, thirst and desire to eat, and to increased satiety and fullness for the remainder of the session (Table 2, VAS, $\mathrm{p}<0.01$ ).

To compare the rewarding value of the food that was chosen within the session, the subjective preference was determined in terms of liking and wanting. Average predetermined relative liking scores for the food items did not differ between the stress and the rest condition, and between the fasted and the satiated (postprandial) condition. However, the quantitative wanting for food, measured as the number of items chosen, was significantly decreased after breakfast (Table 2, rewarding value, $p<0.01$ in the rest condition and $p<0.02$ in the stress condition).

The acute stress as induced by the mathematical test was confirmed with cortisol measurements: cortisol levels were significantly higher, after the unsolvable compared with the solvable math test (Table 2, Cortisol, $p<0.05$ ). Leptin and insulin levels were not significantly increased in the stress condition compared with the rest condition (Table 2) and they were not correlated with cortisol levels.

The effect of stress on hunger and satiety was analyzed from the VAS scores for hunger and satiety. Subjects showed significantly lower scores for satiety after breakfast in the stress condition than in the rest condition, while the scores for hunger were equal in both conditions. The average predetermined liking of the food items chosen during stress was not different from the rest condition (liking). Likewise, the number of food items chosen (wanting) was no different in the stress condition compared with the rest condition. Fat intake was not different during the postprandial second meal compared with breakfast in both the stress and the rest condition (Table 2, n.s.). However, the energy intake, protein intake and carbohydrate intake were not decreased postprandially in the stress condition. Thus carbohydrate and protein intake were relatively higher in the postprandial state (in the absence of hunger) in the stress condition compared with the rest condition. Regarding the food characteristics, firstly subjects selected food items with higher crispiness in the stress versus rest condition (Table 2, interaction effect $p=0.043$ ). Secondly their preference for fullness of taste was decreased postprandially in the rest condition, but not in the stress condition (Table 2, interaction effect $p=0.024$ ) and finally, subjects tended tot choose more energy dense food items in the absence of hunger in the stress condition compared with the rest condition (Table 2, interaction effect $p<0.06)$. Postprandial energy intake was positively correlated with the cortisol levels in the rest condition and in the stress condition $(R=0.697$ and $\mathrm{R}=0.695$, both $\mathrm{p}<0.04$ ).

To determine the effect of choosing food compared with choosing nothing, fMRI data were contrasted accordingly (Figure 2). There was significant activation in the left frontal cortex (possibly Brodmann area 10, $\mathrm{p}<0.05$, FDR corrected). To test the effects of eating breakfast on the representation of food choice related brain activation, fMRI data were contrasted for choosing breakfast versus choosing the second meal. Significantly lower activation was seen in the right putamen and the orbitofrontal cortex of satiated subjects (Figure 3, p<0.05, FDR corrected). 
When comparing the stress condition with the rest condition during breakfast selection, lower activation was present in multiple brain areas: firstly, in the group contrasts, significantly lower activation was observed in the orbitofrontal cortex, frontal cortex and the putamen (Figure 4, FDR $p<0.05$ ). Secondly, ROI analysis of the Amygdala, the cingulate cortex and the hippocampus also showed lower activation in the stress condition compared with the rest condition (Table 2). Finally, the analysis of the putamen ROI revealed that in area of the whole putamen there was significantly reduced activation in the satiated stressed condition, compared with all the other conditions (Table 2).

\section{Discussion}

The main objective of the study was to determine the effect of stress on food choice and food reward. As a starting point, only normal weight women were included. We confirmed that the method we used induced an endocrinological state of moderate acute stress, which was reflected by significantly elevated cortisol levels in the stress condition. Since the effect of stress on food choice and food reward may be mediated by hunger or satiety, we executed the tests in a hunger as well as a satiated condition, both of which were also confirmed by relevant VAS scores.

In terms of the total food choice, energy intake was relatively higher postprandially in the stress condition compared with the rest condition. Additionally, subjects were similarly satiated postprandially both conditions (Table 2, VAS hunger), while satiety was lower in the stress condition compared with the rest condition (Table 2, VAS satiety, interaction effect $p<0.02$ ). This is in line with previous findings on eating in the absence of hunger, where it was found that subjects under stress had a higher energy intake compared with subjects at rest ${ }^{23}$. Nevertheless, liking and wanting were not different under stress compared with rest: we found that the number of food items that were chosen was lower in the satiated condition compared with the fasted condition, while there was no difference between the stress and rest condition. The average relative liking of the food item was not different during stress compared with rest, and in the fasted condition compared with the satiated condition. Therefore, the total rewarding value of the food items that were chosen, which is the combination of liking and wanting ${ }^{24}$, was lower as an effect of satiety but not as an effect of stress.

In contrast to the absent effect of stress on liking and wanting, we found that the food choice under stress compared with rest was different in terms of the food characteristics: subjects chose food items postprandially, that were higher in crispiness and fullness of taste in the stress condition compared with the rest condition. Consequently, the amount of carbohydrates and proteins in the selected food items was relatively higher during the second meal in the stress condition compared with the rest condition. This ultimately led to a trend towards choosing food items with higher energy density in the stress condition compared with the rest condition, resulting in eating in the absence of hunger. Overall, our findings support the idea that stress causes altered food choice, however, it does not lead to increased choice for carbohydrate and fat as proposed before ${ }^{15,17,18,19,20}$, but to increased carbohydrate and protein intake instead. We suggest that the likely cause for this difference is that our study population consisted of normal-weight females, 
while the previous studies were conducted with obese and obesity prone subjects and the difference between the findings may in fact be a key component in the development of obesity. Surprisingly, our present findings indicate that the effects of stress are on food choice are clearly not only present in obese or obesity prone subjects. Furthermore, those effects are not limited to severe stress: our results show the effects of moderate stress that was indicated by significantly elevated cortisol but unaffected leptin and insulin levels.

With respect to relevant brain areas, whole brain contrasts were made and the resulting relevant active areas specific were defined as ROI from those. These regions included the putamen (dorsal striatum), amygdala, hippocampus and cingulate cortex, all of which have been shown to be involved in reward signaling $25,26,27,28,29,30$. Using GLM group contrasts on the fMRI data it was found that reward signaling associated regions were significantly less active while choosing food in the satiated condition compared with the fasted condition (figure 3 ) and while fasted in the stress condition compared with the rest condition (figure 4), respectively. Using ROI event related averages, significantly lower activation was seen in the amygdala, hippocampus and cingulate cortex in the stress condition, compared with the rest condition. Thus stress seems to decrease the sensitivity of the reward system to food cues in general, which is reflected in decreased activation in food reward associated areas.

The shift in food choice towards more crispiness, fullness of taste, and a tendency toward higher energy density in the stressed satiated condition compared with the stressed fasted condition, coincided with a lower activity of the putamen. Since the putamen is also known as a movement related pathway ${ }^{42}$, a contrast for choice for food versus choice for no food while viewing the images was included to confirm that putamen activation did not merely reflect extremity movement signaling (figure 2 ). In this specific contrast, there was a significant activation in the left frontal lobe (possibly Brodmann area 10), which is reportedly involved in integration of different cognitive processes ${ }^{43}$. Therefore the observed effects in the putamen were not due to movement encoding: if moving the joystick and pressing the button in order to choose something did not activate the putamen by itself, then the signal in the other contrasts could not have originated from this motion alone.

Using ROI event related averaging analyses, we found lower activation in the putamen in the combined stress satiated condition. Considering previous evidence that the putamen is involved in reward signaling and reward prediction $27,44,45$, our data suggest that putamen activity may reflect a specific decrease in reward prediction sensitivity: choosing the different sized meals for breakfast and the postprandial second meal, lead similar putamen activation in the rest condition, indicating that the chosen items were predicted as a sufficient meal. In contrast, in the stress condition, the activation in this area was significantly lower during postprandial second meal selection, although the energy content of the selected second meal was not significantly different from the breakfast. Additionally, the striatum has been described to integrate activation from reward pathways and more behavior related pathways ${ }^{46}$. Our data suggests that the putamen integrates the information of hunger and satiety with the predicted food reward to determine the predicted plausibility of the food choice. This prediction was altered in the stress condition, which lead to compensatory food choice toward items with higher energy content. 
Overall, we found that stress interferes with the effects of hunger on energy intake, through lower satiety, a shift in food choice towards food that were higher in carbohydrates, proteins, crispiness and fullness of taste, and that had a tendency to higher energy density on average. This explained relatively higher energy intake in the postprandial state compared with breakfast in this condition. It has been hypothesized that stress may affect food choice. We showed that stress decreased the activation in several reward related brain areas, using GLM contrasts as well as $\mathrm{ROI}$ analyses, and that the putamen may play a complex integrating role in linking reward signals to behavior. Ultimately stress caused higher energy intake, which was attributed to choice for foods with higher carbohydrate and protein content, different structural characteristics and most likely a difference in energy density.

\section{Acknowledgements}

We gratefully acknowledge S. Verhoef for help with the practical work, P. Schoffelen for his contribution regarding the technical realization of the scan paradigm and the synchronization with the scanner, S. Gijsen for essential help and input concerning the fMRI scanner and A. Heinecke for his extensive support for the analyses with BrainVoyager. Furthermore we wish to thank M. Hulsbosch, W. Sluismans and J. Sulon for analyzing the serum and plasma hormone samples.

\section{References}

1 James, P. T., Leach, R., Kalamara, E. \& Shayeghi, M. The worldwide obesity epidemic. Obes Res 9 Suppl 4, 228S-233S (2001).

2 Despres, J. P. \& Lemieux, I. Abdominal obesity and metabolic syndrome. Nature 444, 881-887 (2006).

3 Hutley, L. \& Prins, J. B. Fat as an endocrine organ: relationship to the metabolic syndrome. Am J Med Sci 330, 280-289 (2005).

4 Epel, E. S. et al. Stress and body shape: stress-induced cortisol secretion is consistently greater among women with central fat. Psychosomatic medicine 62, 623-632 (2000).

5 Kyrou, I., Chrousos, G. P. \& Tsigos, C. Stress, visceral obesity, and metabolic complications. Annals of the New York Academy of Sciences 1083, 77-110 (2006).

6 Axelrod, J. \& Reisine, T. D. Stress hormones: their interaction and regulation. Science 224, 452-459 (1984).

7 Eechaute, W., Lacroix, E. \& Leusen, I. [Correlation between the Plasma Level of Free Corticosterone and in Vitro Adrenal Activity in the Rat.]. Archives internationales de physiologie et de biochimie 71, 528-533 (1963).

8 Bjorntorp, P. \& Rosmond, R. Obesity and cortisol. Nutrition 16, 924-936 (2000).

9 Makimura, $\mathrm{H}$. et al. Adrenalectomy reverses obese phenotype and restores hypothalamic melanocortin tone in leptin-deficient ob/ob mice. Diabetes 49, 1917-1923 (2000).

10 Dallman, M. F. et al. Feast and Famine: Critical Role of Glucocorticoids with Insulin in Daily Energy Flow. Frontiers in Neuroendocrinology 14, 303 (1993).

11 Strack, A. M., Sebastian, R. J., Schwartz, M. W. \& Dallman, M. F. Glucocorticoids and insulin: reciprocal signals for energy balance. Am J Physiol 268, R142-149 (1995).

12 la Fleur, S. E. The effects of glucocorticoids on feeding behavior in rats. Physiol Behav 89, 110-114 (2006).

13 la Fleur, S. E., Akana, S. F., Manalo, S. L. \& Dallman, M. F. Interaction between Corticosterone and Insulin in Obesity: Regulation of Lard Intake and Fat Stores. Endocrinology 145, 2174-2185, doi:10.1210/en.2003-1359 (2004).

14 Newman, E., O'Connor, D. B. \& Conner, M. Daily hassles and eating behaviour: the role of cortisol reactivity status. Psychoneuroendocrinology 32, 125-132 (2007).

15 Oliver, G., Wardle, J. \& Gibson, E. L. Stress and food choice: a laboratory study. Psychosomatic medicine 62 853-865 (2000).

16 Wardle, J., Steptoe, A., Oliver, G. \& Lipsey, Z. Stress, dietary restraint and food intake. J Psychosom Res 48, 195-202 (2000).

17 Drewnowski, A., Krahn, D. D., Demitrack, M. A., Nairn, K. \& Gosnell, B. A. Taste responses and preferences for sweet high-fat foods: evidence for opioid involvement. Physiol Behav 51, 371-379 (1992).

18 Elfhag, K. \& Erlanson-Albertsson, C. Sweet and fat taste preference in obesity have different associations with personality and eating behavior. Physiol Behav 88, 61-66 (2006). 
19 Epel, E., Lapidus, R., McEwen, B. \& Brownell, K. Stress may add bite to appetite in women: a laboratory study of stress-induced cortisol and eating behavior. Psychoneuroendocrinology 26, 37-49 (2001).

20 Salbe, A. D., DelParigi, A., Pratley, R. E., Drewnowski, A. \& Tataranni, P. A. Taste preferences and body weight changes in an obesity-prone population. Am J Clin Nutr 79, 372-378 (2004).

21 Berthoud, H. R. Neural control of appetite: cross-talk between homeostatic and non-homeostatic systems. Appetite 43, 315-317 (2004).

22 Westerterp, K. R. \& Speakman, J. R. Physical activity energy expenditure has not declined since the 1980s and matches energy expenditures of wild mammals. Int J Obes 32, 1256-1263 (2008).

23 Rutters, F., Nieuwenhuizen, A. G., Lemmens, S. G. T., Born, J. M. \& Westerterp-Plantenga, M. S. Acute Stressrelated Changes in Eating in the Absence of Hunger. Obesity (2008).

24 Berridge, K. C. Food reward: brain substrates of wanting and liking. Neurosci Biobehav Rev 20, 1-25 (1996).

25 Gottfried, J. A., O'Doherty, J. \& Dolan, R. J. Encoding predictive reward value in human amygdala and orbitofrontal cortex. Science 301, 1104-1107 (2003).

26 O'Doherty, J. P. Reward representations and reward-related learning in the human brain: insights from neuroimaging. Curr Opin Neurobiol 14, 769-776 (2004).

27 Stice, E., Spoor, S., Bohon, C. \& Small, D. M. Relation Between Obesity and Blunted Striatal Response to Food Is Moderated by TaqIAA1 Allele. Science 322, 449-452, doi:10.1126/science.1161550 (2008).

28 Del Parigi, A. et al. Neuroimaging and obesity: mapping the brain responses to hunger and satiation in humans using positron emission tomography. Annals of the New York Academy of Sciences 967, 389-397 (2002).

29 Gautier, J. F. et al. Effect of satiation on brain activity in obese and lean women. Obes Res 9, $676-684$ (2001).

30 Matsuda, M. et al. Altered hypothalamic function in response to glucose ingestion in obese humans. Diabetes 48 , 1801-1806 (1999).

31 Small, D. M., Jones-Gotman, M. \& Dagher, A. Feeding-induced dopamine release in dorsal striatum correlates with meal pleasantness ratings in healthy human volunteers. Neuroimage 19, 1709-1715, doi:S1053811903002532 [pii] (2003).

32 Wang, G. J. et al. Brain dopamine and obesity. Lancet 357, 354-357 (2001).

33 Czyrak, A., Mackowiak, M., Chocyk, A., Fijal, K. \& Wedzony, K. Role of glucocorticoids in the regulation of dopaminergic neurotransmission. Pol J Pharmacol 55, 667-674 (2003).

34 Pani, L., Porcella, A. \& Gessa, G. L. The role of stress in the pathophysiology of the dopaminergic system. Mol Psychiatry 5, 14-21 (2000).

35 Pruessner, J. C., Champagne, F., Meaney, M. J. \& Dagher, A. Dopamine release in response to a psychological stress in humans and its relationship to early life maternal care: a positron emission tomography study using [11C] raclopride. J Neurosci 24, 2825-2831 (2004).

36 Salamone, J. D., Cousins, M. S. \& Snyder, B. J. Behavioral functions of nucleus accumbens dopamine: empirical and conceptual problems with the anhedonia hypothesis. Neurosci Biobehav Rev 21, 341-359 (1997).

37 Fulton, S., Richard, D., Woodside, B. \& Shizgal, P. Interaction of CRH and energy balance in the modulation of brain stimulation reward. Behav Neurosci 116, 651-659 (2002).

38 Stunkard, A. J. \& Messick, S. The three-factor eating questionnaire to measure dietary restraint, disinhibition and hunger. J Psychosom Res 29, 71-83 (1985).

39 Peters, M. L. et al. Cardiovascular and endocrine responses to experimental stress: effects of mental effort and controllability. Psychoneuroendocrinology 23, 1-17 (1998).

40 Lemmens, S. G. T. et al. Eating what you like induces a stronger decrease of 'wanting' to eat. Physiology \& Behavior 98, 318-325, doi:10.1016/j.physbeh.2009.06.008 (2009).

41 Talairach, J. \& Tournoux, P. Co-planar Stereotaxic Atlas of the Human Brain. (Thieme, 1988).

42 Schultz, W., Apicella, P., Scarnati, E. \& Ljungberg, T. Neuronal activity in monkey ventral striatum related to the expectation of reward. J. Neurosci. 12, 4595-4610 (1992).

43 Ramnani, N. \& Owen, A. M. Anterior prefrontal cortex: insights into function from anatomy and neuroimaging. Nat Rev Neurosci 5, 184-194, doi:10.1038/nn1343

nrn1343 [pii] (2004).

44 Schultz, W. Reward Signaling by Dopamine Neurons. Neuroscientist 7, 293-302 (2001).

45 Haruno, M. \& Kawato, M. Different Neural Correlates of Reward Expectation and Reward Expectation Error in the Putamen and Caudate Nucleus During Stimulus-Action-Reward Association Learning. J Neurophysiol 95, 948-959, doi:10.1152/jn.00382.2005 (2006).

46 Hollerman, J. R. et al. in Progress in Brain Research Vol. 126 193-215 (Elsevier, 2000). 
Chapter 5

\section{High HPA-axis activation disrupts the link between liking and wanting with liking and wanting related brain signaling}

Jurriaan M Born, Mieke JI Martens, Femke Rutters, Sofie GT Lemmens, Rainer Goebel, and Margriet S Westerterp-Plantenga,Physiol Behav. 2011 Aug 23;105(2): 


\section{Abstract}

Background: Eating behavior changes under stress, i.e. during high HPA-axis activation.

Aim: Assessment of effects of high versus low HPA-axis activation on liking and wanting related brain signaling in relevant regions.

Methods: 15 female subjects $(21.5 \pm 0.4 \mathrm{y}, \mathrm{BMI}=22.2 \pm 0.4)$ completed fMRI scans on two days, in a fasted as well as a satiated condition on each day. The days were sorted by HPA-axis activation, resulting in two sufficiently separated HPA-axis states which was statistically confirmed $(p<.05)$. During scans, subjects rated liking and wanting for food images; wanting indicated food choice for the subsequent meal. Energy-intake, hunger and fullness were additionally recorded.

Results: Hunger changed significantly over the meal $(p<.001)$. Energy intake was lower during the second meal $(p<.001)$. Behavioral wanting was lower after breakfast $(p<.01)$, behavioral liking did not change. During low HPA-activation, liking task related signaling (TRS) pre-meal in the anterior insula predicted behavioral liking, wanting TRS in the anterior insula, nucleus accumbens and thalamus predicted behavioral wanting. During high HPA-activation, these relationships were not present pre-meal, but post-meal behavioral liking was predicted in the nucleus accumbens and wanting in the caudate.

Conclusion: High HPA-axis activation disrupted and redirected the connection of behavioral liking/wanting with the specifically associated brain signaling in relevant regions. 


\section{Introduction}

The prevalence of overweight, obesity and the associated co-morbidities are increasing in the developed world ${ }^{1,2}$. The main cause of this increase is a positive energy balance that is maintained for extended periods of time ${ }^{3}$. Food choice and energy intake has been attributed to the rewarding value of food, and this rewarding value was defined as a combination of liking and wanting food ${ }^{4}$. Additionally, overeating has previously been partially attributed to stress indicated by activation of the hypothalamus-pituitary-adrenal axis (HPA-axis) and increased levels of cortisol in the blood ${ }^{5,6}$. This HPA-axis activation seems to results in food choice for highly rewarding food items ${ }^{7,8}$. We previously showed that acute stress lead to increased behavioral wanting for snacks and sweet foods in overweight subjects, yet decreased behavioral wanting in normal weight subjects during a postprandial second meal ${ }^{9}$. Additionally, food choice during relatively high (compared with relatively low) HPA-axis activation, was changed towards items that were crispy and full of taste, a higher energy intake, and lower striatal signaling during a second meal ${ }^{10,11}$.

In these studies, it remained unclear how behavioral liking and wanting tasks are linked to the related brain signaling, and subsequently, how this link is influenced by HPA-axis activation. We hypothesized that the link between behavioral liking and wanting and signaling in relevant brain areas may be disturbed during high versus low HPA-activation, helping to explain altered motivation towards eating behavior during stress.

\section{Methods}

\section{Subjects:}

The study was approved by the Medical Ethical Committee of Maastricht University. Informed, written consent was obtained from all subjects. Fifteen healthy, righthanded test subjects were included (age $=21.5 \pm 0.4$, bMl $=22.2 \pm 0.2$, range 19.5-25.5.

\section{fMRI sessions:}

Subjects came twice, on two separate days, to the university in the morning, after an overnight 10 hour fast, which was confirmed individually. Days were assigned to high or low HPA-axis activity, within subjects, based upon the averaged cortisol concentrations during $4 \mathrm{~h}$ in the morning. This separation was clear and statistically significantly confirmed. Subjects arrived in the fasted state and participated in the first fMRI run. The satiated state was achieved by eating breakfast. Fullness was confirmed and the measurements were repeated. To obtain blood for hormone samples, a cannula was placed in the antecubital vein for the duration of the session. Following each set of questionnaires, blood was taken. The samples were transferred into a serum tube (BD Vacutainer with separation gel, $8.5 \mathrm{~mL}$, BD Biosciences, Franklin Lakes, NJ, USA), serum cortisol was obtained by centrifugation (4C, 3000 r.p.m., $10 \mathrm{~min}$ ). Samples were stored at $-80 \mathrm{C}$ until analysis using one radio immuno assay (Département des Sciences Fonctionnelles, B41 Physiologie de Reproduction, Liège, Belgium). The assay variability was V.C. $<5 \%$. Average cortisol concentrations were determined per subject, per day. To compare 
days with states of high versus low HPA-axis activation, each subjects' days were assigned to the high versus low cortisol level based upon the average $4 \mathrm{~h}$ cortisol measurements, resulting in a significantly separated HPA-axis low as well as high condition within each subject (Table 1, $p<0.05$ ).

During the session four questionnaires and two fMRI scans were taken during which the subsequent meals (breakfast and second meal) were chosen by average

\section{Table 1:}

Behavioral measurements, hormone measurements and relations between behavioral wanting and wanting TRS in specified regions, dependent on HPA-axis low and high conditions.

\begin{tabular}{|c|c|c|c|c|c|}
\hline & \multicolumn{2}{|c|}{ HPA Low } & \multicolumn{2}{|c|}{ HPA High } \\
\hline & & $\begin{array}{c}\text { Pre-meal / } \\
\text { Meal } 1\end{array}$ & $\begin{array}{c}\text { Post-meal I } \\
\text { Meal } 2\end{array}$ & $\begin{array}{c}\text { Pre-meal I } \\
\text { Meal } 1\end{array}$ & $\begin{array}{c}\text { Post-meal I } \\
\text { Meal } 2\end{array}$ \\
\hline \multirow{7}{*}{\multicolumn{2}{|c|}{$\begin{array}{r}\text { Wanting (AU) } \\
\text { Liking (AU) } \\
\text { El (MJoule) } \\
\text { Cortisol 4h average (nmol/l) } \\
\text { Cortisol intercept (nmol/l) } \\
\text { Hunger (mmVAS) }\end{array}$}} & \multicolumn{4}{|c|}{ Mean $\pm S E M$} \\
\hline & & $2.16 \pm 0.10$ & $1.59 \pm 0.13 a$ & $2.25 \pm 0.06$ & $1.66 \pm 0.10 \mathrm{a}$ \\
\hline & & $2.70 \pm 0.07$ & $2.64 \pm 0.07$ & $2.66 \pm 0.06$ & $2.56 \pm 0.06$ \\
\hline & & $2.81 \pm 0.26$ & $0.91 \pm 0.27 a$ & $2.80 \pm 0.15$ & $0.55 \pm 0.17 a$ \\
\hline & & \multicolumn{2}{|c|}{$275.89 \pm 23.93$} & \multicolumn{2}{|c|}{$346.96 \pm 42.49 \mathrm{~b}$} \\
\hline & & \multicolumn{2}{|c|}{$382.68 \pm 29.10$} & \multicolumn{2}{|c|}{$432.19 \pm 39.98 b$} \\
\hline & & $73.0 \pm 4.4$ & $18.5 \pm 3.4 a$ & $74.8 \pm 3.4$ & $18.3 \pm 4.03 a$ \\
\hline \multirow{2}{*}{\multicolumn{2}{|c|}{ Fullness (mmVAS) }} & $13.0 \pm 1.5$ & $64.7 \pm 4.0 \mathrm{a}$ & $14.0 \pm 2.6$ & $63.1 \pm 4.4 a$ \\
\hline & & \multicolumn{4}{|c|}{ Correlations $\left(R^{2}\right)$} \\
\hline \multirow{4}{*}{$\begin{array}{r}\text { Wanting } \\
\text { related to } \\
\text { Wanting TRS } \\
\left(\mathbf{R}^{2}\right)\end{array}$} & Insula & $0.43^{*}$ & 0.00 & 0.00 & 0.00 \\
\hline & Caudate & 0.22 & 0.00 & 0.00 & $0.52^{*}$ \\
\hline & N. Accumbens & $0.29^{*}$ & 0.00 & 0.00 & 0.00 \\
\hline & Thal & $0.29^{*}$ & 0.00 & 0.00 & 0.00 \\
\hline \multirow{4}{*}{$\begin{array}{r}\text { Liking related } \\
\text { to Liking TRS } \\
\left(R^{2}\right)\end{array}$} & Insula & $0.35^{*}$ & 0.00 & 0.00 & 0.00 \\
\hline & Caudate & 0.25 & 0.00 & 0.00 & 0.00 \\
\hline & N. Accumbens & 0.00 & 0.00 & 0.00 & $0.33^{*}$ \\
\hline & Thal & 0.25 & 0.00 & 0.00 & 0.00 \\
\hline
\end{tabular}

a Significant at $p<.05$ vs. Pre-meal, $b$ significant at $p<.05$ vs. HPA Low, * significant correlation.

wanting rating $(\geq 2.5)$ on a four button box; before breakfast in a fasted, after breakfast in a satiated condition. The hungry pre-meal and satiated state post-meal were met, indicated by high pre-meal versus low post-meal ratings for hunger, as well as low pre-meal versus high post-meal ratings of fullness (Table 1, $p<.001$ ). The fasted and satiated conditions were identical and therefore directly comparable.

Subjects knew all items from a previous food tasting and texture rating session. The functional MRI run included four segments, of each wanting and liking. Segments: instruction $12 \mathrm{~s}$, fixation $12 \mathrm{~s}$, reminder $0.5 \mathrm{~s}$, food image $2 \mathrm{~s}$, fixation $2-5 \mathrm{~s}$, rating period (1.5s), inter-trial interval, $7-9 \mathrm{~s}$; total duration $\sim 35 \mathrm{~min}$.

BOLD images were taken continuously $\left(T R=2 s, T E=26 \mathrm{~ms}\right.$, Flip angle $=90^{\circ}$, matrix=96x96, FOV=269 voxel size $2.8 \times 2.8 \times 2.6 \mathrm{~mm}$, gap $0.2 \mathrm{~mm}$ ).

The effect of the meals was assessed four times with $100 \mathrm{~mm}$ visual analog scales (VAS) for hunger and fullness: before and after each scan. Imaging data was extracted using BrainVoyagerQX 2.2 with standard procedures (Brainlnnovation B.V., Maastricht, The Netherlands 12,13) and temporal high pass filtering with a 

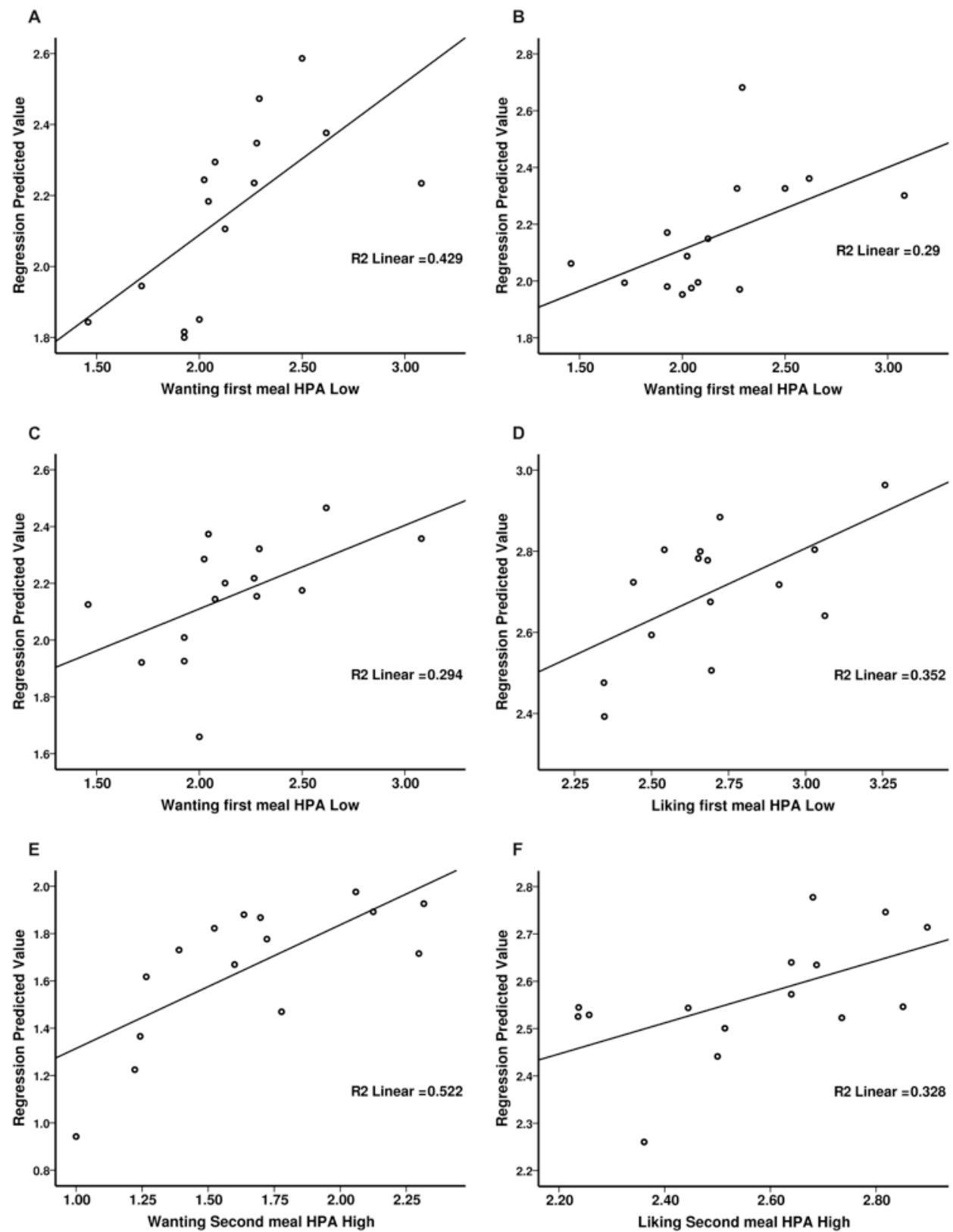

Figure 1 Multiple regression analyses of behavioral liking and wanting versus liking and wanting TRS, respectively.

Indicated trends are significant at $p<0.05$. Models shown are HPA- axis low condition: behavioral wanting predicted by A: left anterior insula, B: left nucleus accumbens, C: left thalamus; behavioral liking predicted by D: bilateral anterior insula. HPA-high condition: behavioral wanting predicted by E: bilateral caudate; behavioral liking predicted by F: bilateral nucleus accumbens. 
6cycles window. Predictors for 'low liking', 'liking', 'low wanting' and 'wanting' of the food image stimuli were created using the subjects' inputs during the scan. Linear habituation was modeled using BVA-Predictor tool software (J.M.Born, Maastricht). Anatomical regions of interest (ROI), including regions associated with homeostatic signaling and taste, or reward, had been indicated for liking and wanting related processes previously ${ }^{4,14}$. From these regions, those regions that were identified in liking and wanting related signaling in humans in our previous study were included in the analyses here. These regions were the anterior insula, caudate, putamen, nucleus accumbens and amygdala ${ }^{10}$. Parameter (signal) estimates for 'liking' and 'wanting' were extracted using ROI ANOVA for model fitting. Shapiro-Wilk testing was used to confirm normal data distribution and multiple regressions between behavioral liking and wanting ratings and bilateral fMRI ROI activation data high liking or high wanting) were performed using PASWStatistics 18.

\section{Results and discussion}

In both HPA-axis conditions, behavioral wanting decreased similarly and significantly over the meal (Table 1, $p<.001$ ), while there was no significant change in behavioral liking (Table 1, $p=.07$ ). These findings agree with our previous studies, in which we showed that wanting was generally more variable over meals and conditions compared with liking ${ }^{11,13,15}$. The absence of a significant effect of HPA-axis activation on behavioral liking and wanting, and energy intake in our normal weight subjects, agrees with our study that showed changes wanting specifically in overweight versus normal weight subjects during stress ${ }^{9}$. During both second meals, energy intake was significantly lower than during the breakfast before (Table 1, $p<.001$ ), thus energy intake was supported by hunger, fullness and wanting, according to the subjects ratings.

Concerning the link between behavioral data and brain activation, pre-meal in HPAlow conditions, wanting TRS in the left anterior insula, nucleus accumbens and thalamus predicted behavioral wanting (Figure $1 \mathrm{~A}-\mathrm{C}$ and Table 1, $\mathrm{p}<.05$ ) and liking TRS in the bilateral anterior insula predicted behavioral liking (Figure 1D and Table $1, p<.02)$. This indicates that behavioral wanting was supported by wanting TRS in regions related to reward, taste and integrative processes, while behavioral liking was supported by taste related regions ${ }^{11,16}$, confirming that liking and wanting are related, but distinct processes ${ }^{4}$.

Post-meal no relations were found between behavioral and imaging data in the HPAlow conditions (Table 1). In contrast, during HPA-high conditions, pre-meal all relations between behavioral liking and wanting were absent (Table 1), while postmeal behavioral wanting was predicted by wanting TRS in the caudate (Figure 1E and Table 1, p<.02) and behavioral liking was predicted by liking TRS in the left nucleus accumbens (Figure $1 \mathrm{~F}$ and Table $1, \mathrm{p}<.03$ ). Thus in HPA-low conditions, experiencing food intake seems to reduce the link between specific brain regions and behavioral liking and wanting. In contrast, in HPA-high conditions, no significant relationship was found pre-meal while different associations between behavioral liking and wanting associated TRS appeared post meal, compared with HPA-low conditions. This suggests that HPA-axis activation may redirect reward sensitivity in the brain and reduce the implicit perception of the first reward exposure during high 
HPA-activation, which could help to explain why subjects are prone to overeating during stress, as it has been reported in overweight subjects ${ }^{9,17}$.

Post-meal in the HPA-high conditions, links between behavioral liking and wanting and TRS, surprisingly appeared in different areas compared with HPA-low conditions: Only one region was involved during HPA-high conditions, namely the caudate (Table 1). Possibly, the reduced number of regions reflects moderate reward deficiency with an increased need to pursue reward ${ }^{17}$. Liking support shifted from the anterior insula, a region known for taste perception and hedonic signaling, to the nucleus accumbens which has been implicated in reward and motivational processes ${ }^{16,18,19}$ and which was related to wanting in HPA-low conditions (Figure 1B). Thus in HPA-high conditions, liking appears connected to processes that are related to wanting processes in HPA-low conditions.

In conclusion, energy intake reflected hunger, fullness and behavioral wanting. In HPA-low conditions, behavioral liking and wanting were supported by liking and wanting TRS in the anterior insula, nucleus accumbens and thalamus. In HPA-high conditions, the TRS supporting behavioral liking and wanting pre-meal was not significant, while it appeared in more reward related regions post-meal. Thus high HPA-axis activation may disrupt and redirect normal liking and wanting related brain signaling, possibly explaining stress induced eating in obesity prone subjects.

\section{References}

1 Seidell, J. C. Epidemiology and health economics of obesity. Medicine 34, 506-509 (2006).

2 Catenacci, V. A., Hill, J. O. \& Wyatt, H. R. The Obesity Epidemic. Clinics in Chest Medicine 30, 415-444 (2009).

3 Pietilainen, K. H. et al. Inaccuracies in food and physical activity diaries of obese subjects: complementary evidence from doubly labeled water and co-twin assessments. Int J Obes 34, 437-445 (2010).

4 Berridge, K. C. Food reward: brain substrates of wanting and liking. Neurosci Biobehav Rev 20, 1-25 (1996).

5 Axelrod, J. \& Reisine, T. D. Stress hormones: their interaction and regulation. Science 224, 452-459 (1984).

6 Eechaute, W., Lacroix, E. \& Leusen, I. [Correlation between the Plasma Level of Free Corticosterone and in Vitro Adrenal Activity in the Rat.]. Archives internationales de physiologie et de biochimie 71, 528-533 (1963).

7 Oliver, G., Wardle, J. \& Gibson, E. L. Stress and food choice: a laboratory study. Psychosomatic medicine 62, 853-865 (2000).

8 Wardle, J., Steptoe, A., Oliver, G. \& Lipsey, Z. Stress, dietary restraint and food intake. J Psychosom Res 48, 195-202 (2000).

9 Lemmens, S. G., Rutters, F., Born, J. M. \& Westerterp-Plantenga, M. S. Stress augments food 'wanting' and energy intake in visceral overweight subjects in the absence of hunger. Physiology \& Behavior In Press, Uncorrected Proof (2010).

10 Born, J. M., Lemmens, S. G. T., Formisano, E., Goebel, R. \& Westerterp-Plantenga, M. S. Differences between liking and wanting signals in the human brain and relations with BMI and cognitive dietary restraint. Am J Clin Nutr (2011).

11 Born, J. M. et al. Acute stress and food-related reward activation in the brain during food choice during eating in the absence of hunger. Int J Obes 34, 172-181 (2010).

12 Goebel, R., Esposito, F. \& Formisano, E. Analysis of functional image analysis contest (FIAC) data with brainvoyager QX: From single-subject to cortically aligned group general linear model analysis and selforganizing group independent component analysis. Human Brain Mapping 27, 392-401, doi:10.1002/hbm.20249 (2006).

13 Born, J. M., Lemmens, S. G. T., Formisano, E., Goebel, R. \& Westerterp-Plantenga, M. S. Differences between liking and wanting signals in the human brain and relations with cognitive dietary restraint and body mass index. Am J Clin Nutr In press, doi:doi:10.3945/ajcn.111.012161 (2011).

14 Berthoud, H. R. Homeostatic and non-homeostatic pathways involved in the control of food intake and energy balance. Obesity (Silver Spring) 14 Suppl 5, 197S-200S (2006).

15 Lemmens, S. G. T. et al. Eating what you like induces a stronger decrease of 'wanting' to eat. Physiology \& Behavior 98, 318-325, doi:10.1016/j.physbeh.2009.06.008 (2009).

16 Berthoud, H.-R. Multiple neural systems controlling food intake and body weight. Neuroscience \& Biobehavioral Reviews 26, 393-428 (2002). 
17 Blum, K. et al. Reward deficiency syndrome: a biogenetic model for the diagnosis and treatment of impulsive, addictive, and compulsive behaviors. J Psychoactive Drugs 32 Suppl, i-iv, 1-112 (2000).

18 Knutson, B., Adams, C. M., Fong, G. W. \& Hommer, D. Anticipation of Increasing Monetary Reward Selectively Recruits Nucleus Accumbens. J. Neurosci. 21, 159RC- (2001).

19 Di Chiara, G. et al. Dopamine and drug addiction: the nucleus accumbens shell connection. Neuropharmacology 47, 227 (2004). 
Chapter 6

\section{Shifts in protein versus carbohydrate intake differentially affects liking and wanting related \\ brain signaling}

Jurriaan M Born, Mieke JI Martens, Sofie GT Lemmens, Rainer Goebel, and Margriet S Westerterp-Plantenga, submitted for publication. 


\section{Abstract}

Background: Extreme macronutrient intakes lead to subsequent macronutrient compensation, possibly accompanied by different brain signaling.

Aim: Determine effects of ingesting a high protein versus high carbohydrate diet on liking and wanting task related brain signaling (TRS) in relevant regions and subsequent macronutrient and energy intake.

Methods: 30 female subjects (mean $\pm S D$ 21.6 $\pm 2.2 \mathrm{y}, \mathrm{BMI}=25.0 \pm 3.7$ ) completed four fMRI scans: two fasted and two satiated on two different days. During scans, subjects rated all food items for liking and wanting, thereby choosing the subsequent meal. High protein versus high carbohydrate conditions were generated using a protein $(P)$, or carbohydrate $(C)$ drink at the first meal. Energy-intake and hunger were recorded.

Results: High protein $(P=53.7 \pm 2.1 \mathrm{En} \%, C=6.4 \pm 1.3 \mathrm{En} \%)$ and high carbohydrate conditions $(P=11.8 \pm 0.6 \mathrm{En} \%, C=70.0 \pm 2.4 \mathrm{En} \%)$ were achieved. Hunger, energy intake, and behavioral liking and wanting ratings were decreased after the first meal $(p<0.001)$. Comparing the first meal with the second meal, macronutrient content shifted: C $-26.9 \mathrm{En} \%$ in the $\mathrm{C}$ condition, versus $\mathrm{P}-37.8 \mathrm{En} \%$ in the $\mathrm{P}$ condition. After The First Meal in the $C$ condition, wanting TRS was increased in the hypothalamus. After The First Meal in the P condition, liking TRS was decreased in the putamen $(p<0.05)$.

The change in Energy intake from the first meal to the second meal, was inversely related to the change in liking TRS in the striatum and hypothalamus in the carbohydrate condition and positively related in the protein condition $(p<0.05)$.

Conclusion: wanting task related signaling and liking task related signaling were affected differentially after a shift in carbohydrate or protein intake, underscoring subsequent energy intake and shift in macronutrient composition. 


\section{Introduction}

The prevalence of obesity and its co-morbidities is increasing in the developed world $^{1,2}$. This is caused by energy-intake exceeding energy expenditure, i.e. positive energy balance ${ }^{3}$ and may be influenced by features of food intake, such as eating in the absence of hunger ${ }^{4}$. This overeating is often driven by reward seeking ${ }^{5}$. Previous studies suggested that eating palatable food may disrupt food choice, leading to higher energy intake ${ }^{6}$. This increase in food intake was related to BMI and palatability ${ }^{7}$. Additionally, evidence was found that glucose intake may bias neurological processes toward higher energy intake $e^{8,9,10}$. Conversely, proteins have been shown to assist in weight loss and weight maintenance, by increasing satiety over a meal and decreasing energy intake ${ }^{11,12}$. However, compliance to protein diets is relatively low ${ }^{11}$, and little is known about the rewarding value of proteins and their influence on brain signaling. To study the rewarding value of food, we recently used Berridge's model that describes the rewarding value of food as 'liking', the hedonic preference for, or palatability of a food item, and 'wanting', the motivation to eat a particular food item ${ }^{13}$. Energy-intake is mainly determined by wanting 5,14 , while liking is relatively more stable ${ }^{14}$. Additionally, we showed that liking and wanting are signaled in several overlapping, as well as specific areas in the human brain ${ }^{14}$. We observed that wanting task related signaling (TRS) was represented significantly in the hypothalamic region and the striatum and that signaling in the satiated condition in these regions was typically inversely related with $\mathrm{BMI}^{14}$. Additionally, we observed liking task related signaling in the insula, and when related to cognitive dietary restraint also in the nucleus accumbens ${ }^{14,15}$. Here we assessed the way in which proteins versus carbohydrates may affect liking and wanting related signaling in the human brain in the short term, using functional MRI. We hypothesized, that protein intake may acutely modulate hypothalamic and striatal signaling differently from carbohydrate intake, thereby differentially affecting brain signaling and motivation for food selection and energy intake in the absence of hunger.

\section{Subjects and methods}

\section{Subjects}

This study was approved and registered under MEC 09-3-035 by the Medical Ethical Committee of Maastricht University and at the Netherlands Trial Register under NTR1904. Subject recruitment started on November 23rd 2009 and included subjects with $\mathrm{BMI}=19-33$. 35 test subjects were screened and informed, written consent was obtained from all subjects. Inclusion criteria were: healthy female subjects, body weight ranging from normal weight to obese (BMI 19-33) and righthandedness. Exclusion criteria were a personal or familial history of psychiatric disorder, recent dieting, or the use of intra-uterine contraceptives other than Mirena. Thirty subjects with the following characteristics were included (Mean $\pm S D)$ : age $=$ 21.6 $\pm 2.2, \mathrm{BMI}=25.0 \pm 3.7$, range 19.4-32.8.

\section{Conditions}

Two conditions were generated using two macronutrient specific drinks that were designed to be matched for consistency, energy content, taste and pleasantness of taste: A high carbohydrate $(\mathrm{C})$ condition was generated by supplementing the first 
meal with a drink high in carbohydrates (Carbohydrates (C): 94 En\%, Protein $(P): 6$ En\%, Fat $(F)$ : 0 En\%) and a high protein condition $(P)$ was generated by supplementing the first meal with a drink high in Protein (C: $10 \mathrm{En} \%, \mathrm{P}: 87 \mathrm{En} \%$, F: $3 \mathrm{En} \%$ ). The carbohydrate drink contained $265.2 \mathrm{~kJ}$ and consisted of: $22.5 \mathrm{~g}$ Optimel yoghurt (Friesland Campina, Amersfoort, the Netherlands), $2.5 \mathrm{~g}$ strawberry syrup (PLUS, PLUS holding, de Bilt, the Netherlands), 10.0g Fantomalt (Nutricia, Zoetermeer, the Netherlands), 2.5 Nutilis (Nutricia, Zoetermeer, the Netherlands), $0.1 \mathrm{~g}$ Canderel powdered sweetener (Pietercil Barends, Zoetermeer, the Netherlands) and $62.5 \mathrm{~g}$ water. $100 \mathrm{~g}$ of the protein drink contained $249.5 \mathrm{~kJ}$ and contained: $10.0 \mathrm{~g}$ Perfect protein 85+ Strawberry (Perfect Body, Almere, the Netherlands), 2.5g Perfect protein 95 (Perfect Body, Almere, the Netherlands), 25g yoghurt ("Magere kwark", PLUS holding, de Bilt, the Netherlands), and $62.5 \mathrm{~g}$ water. The amount of each drink given was individually determined as $14 \%$ of daily energy requirement (DER). DER was estimated using the Harris-Benedict equation to calculate the basal metabolic rate $(B M R)$ and the the activity index (AI) was assessed using the Baecke questionnaire 16,17. DER was determined as BMR $\mathrm{x}$ AI. The drinks were given five minutes before the first meal had to be consumed completely by then end of the meal.

\section{Functional MRI sessions}

A fasted and satiated condition was created: Subjects came fasted to the university Subjects were instructed not to drink any alcoholic beverages the day before the test and not to eat, or drink coffee or tea after 10 p.m. the evening before testing.

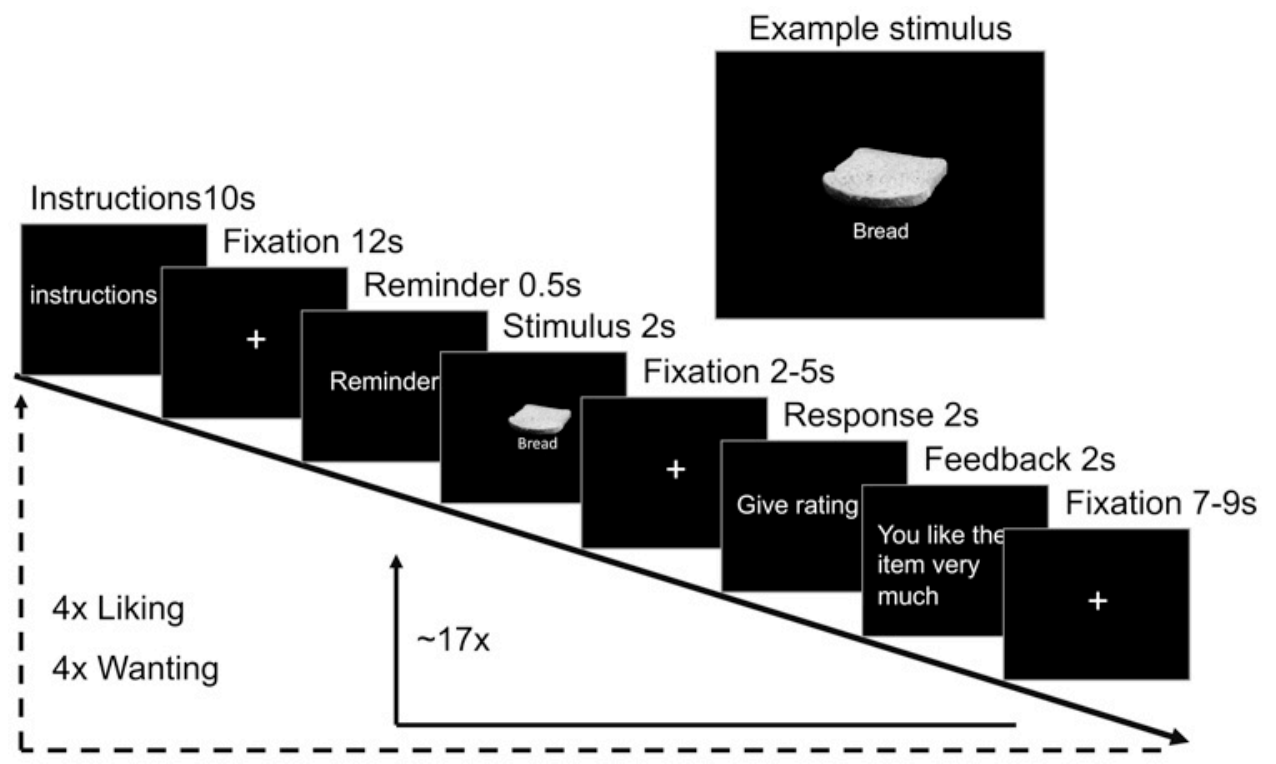

Figure 1: An overview of the functional MRI paradigm.

Each fMRI session took approximately 45 minutes including a 35-minute functional MRI run. Example stimulus: Each food image was shown centered with the food name below the image. Images were standardized for size and color. B: Four segments of each liking and wanting were shown (dotted line) in counterbalanced order. Segments contained $\sim 17$ event related stimulus trials (solid line). 
Thereby, subjects were fasted for at least 10 hours. Adherence to these instructions was individually confirmed. Each session included four Visual analog scale (VAS) questionnaires for hunger and satiety and two fMRI scans during which the subsequent meal was chosen ${ }^{14}$. The VAS questionnaires consisted of $100 \mathrm{~mm}$ lines, anchored with "not at all" at the far left "extremely" at the far right and questions asked were "How hungry are you?" and "How full do you feel?". The satiated condition was created by eating the first meal between the first and the second fMRI scan. The second meal followed the second scan. Both meals consisted of the food items that were chosen from 13 food images within the scanner. Each meal was offered immediately after the scan. The food items that were used had been tasted individually and rated for their taste and texture on a separated day. In addition, the $\mathrm{C}$ and $\mathrm{P}$ drinks were tasted and their similarity in hedonic value and texture was individually confirmed. After tasting, all items were known to the subjects ${ }^{14}$. The food images were presented against a black background (Figure 1A). All items were resized to match for intensity by the subjects during the tasting session. To account for the limited number of different items, all items were given in large quantities. Subjects were not required to eat all that was offered, but some of each chosen item had to be consumed. The setup and paradigm during the fasted and satiated state were identical and therefore directly comparable in the analyses.

\section{Functional MRI settings and paradigm}

The subjects were scanned in a Siemens Magnetom Allegra magnetic resonance imaging system (Siemens, Erlangen, Germany), with the standard one-channel head coil. T1 weighted scout images were used to confirm the subjects' position. Four segments of wanting and four segments of liking were presented during the functional run. The food images were shown one by one in an event related design (Figure $1 \mathrm{~A}$ and $\mathrm{B}$ ), thus presented one by one and the subjects were asked to rate each for 'liking' and 'wanting'14. Berridge's model for the rewarding value of food was used i.e. liking and wanting ${ }^{13}$. Thus behavioral liking and wanting was assessed using the ratings obtained in the scanner. Here, the question to determine liking was "how much do you like this item, not considering if you want to eat it right now?". Wanting was determined using the question "how much do you want to eat this item right now?", while the subjects were instructed that the items that were rated high in wanting had to be consumed immediately after the scan. 'Liking' thereby represented explicit food preference and 'wanting' represented explicit food choice. In total, the functional run took $\sim 35 \mathrm{~min}$ and each image was shown 4 to 5 times. A four-button response box was used to obtain the ratings (LUMltouch, Photon Control, Burnaby, BC, Canada) using the right hand. The buttons represented a value of 1-4. The average ratings were calculated directly after the scan and items rated 2.5 or higher were offered to eat. Functional MRI images were acquired throughout the session using a $\mathrm{T}^{*}$ weighted protocol to obtain blood oxygen level dependent $(\mathrm{BOLD}) \mathrm{T}^{*}$ signal $\left(\mathrm{TR}=2 \mathrm{~s}, \mathrm{TE}=26 \mathrm{~ms}\right.$, Flip angle $=90^{\circ}$, matrix $=$ $96 \times 96$, field of view $=269$, voxel size $3.0 \times 3.0 \times 2.9 \mathrm{~mm}$, gap $0.1 \mathrm{~mm}$ resulting in a functional voxel size of $27 \mathrm{~mm}^{3}$ ).

\section{fMRI data preprocessing}

All fMRI data were imported, aligned and analyzed using BrainVoyager QX version 2.3 (Brain Innovation B.V., Maastricht, The Netherlands). Preprocessing was 
performed using the standard methods in Brainvoyager $Q X^{14}$. To align all functional images, auto alignment (6 parameter affine) to each subjects' own $1 \mathrm{~mm}$ isovoxel high resolution T1 weighted anatomical scan was performed and all images were transformed into the Talairach coordinate system (Talairach and Tournoux, 1988). The resulting resolution was $3 \times 3 \times 3 \mathrm{~mm}$, i.e. a functional voxel size of $27 \mathrm{~mm} 3$.

\section{Region of interest definition}

Anatomical regions of interest (ROI) related to our hypotheses were determined from our earlier study and the ROI definitions files from that study were used ${ }^{14}$. Exploratory analyses were used to identify regions to be included and included regions were bilateral caudate, nucleus accumbens, putamen and hypothalamic region.

\section{Behavioral measurements}

Visual analog scales for hunger and satiety were taken four times to determine effects of a pre-meal (hunger) and a state after a meal (absence of hunger). These questionnaires were obtained before and after each scan. Thus the first meal was eaten in between questionnaire 2 and 3 , the second meal was eaten after questionnaire 4 . In addition, the liking and wanting rating that was given for each item was recorded and analyzed.

\section{Data analysis}

Brain activation was analyzed using a GLM approach with separate predictors for liking and wanting: Using the subjects' image ratings, each image was ranked according to its average rating. The five highest and five lowest images were selected to avoid an unbalanced design. Consequently, the predictors obtained were 'low liking', 'high liking', 'low wanting' and 'high wanting'. Each trial was defined as the duration of the image presentation and was modeled using the standard canonical two-gamma hemodynamic response function (2gHRF) and an additional predictors were created to contain the non-selected trials. Furthermore, several

\section{Table 1:}

Correlation coefficients for the relationship between wanting task related signaling in the specified regions and BMI with a linear and quadratic model, and related to the average wanting rating for the trials included in the analysis.

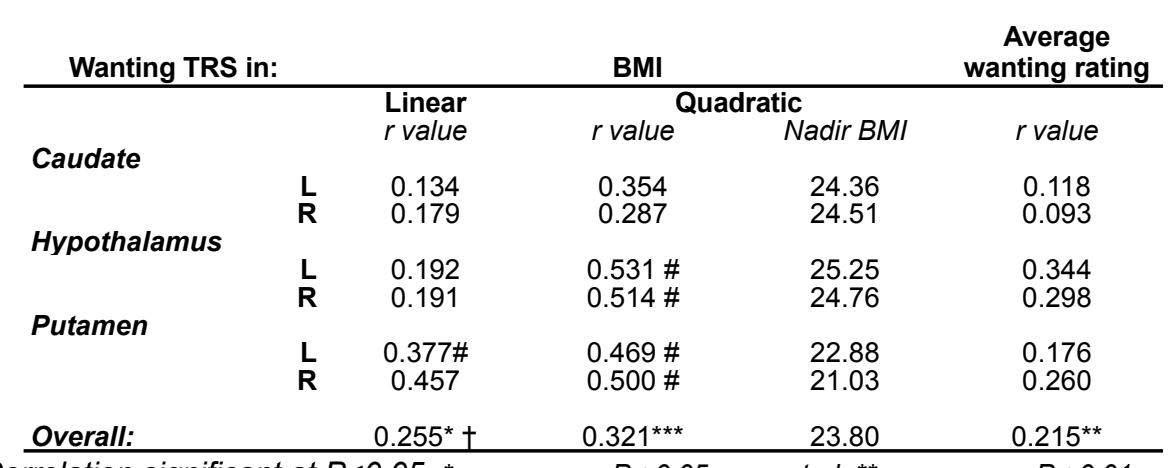

\# Correlation significant at $P<0.05$, ${ }^{*}$ versus zero $P<0.05$, corrected, ${ }^{* *}$ versus zero $P<0.01$, corrected, ${ }^{* * *}$ versus zero $P<0.005$, corrected, + Correlations linear $<$ quadratic $P<0.02$ 
confound predictors were included: Motion estimates obtained in the motion correction step of functional data preprocessing were de-trended, z-normalized and high-pass filtered (cutoff $0.025 \mathrm{~Hz}$ ) using the Brainvoyager Analysis-Predictor tool (BVA-Predictor Tool, J.M. Born, Maastricht), and subsequently a multi-subject GLM was run using these transformed motion estimates on the subjects' data. The three strongest predictors (group average) were included: $y$ and $z$ translation and $x$ rotation. Linear habituation was modeled as a single predictor from all model parameters (BVA-Predictor Tool). Additionally, onsets of instructions, reminder and response request were modeled using the $2 \mathrm{gHRF}$ to account for the variation associated with these visual stimuli. Dummy coding was used to extract group effects for pre-meal and after the first meal and carbohydrate vs. protein conditions (using BVA-Predictor Tool). From regions of interest, parameter estimates for the wanting task related models were extracted and baseline corrected, in which baseline represents the parameter estimates for the constant in the GLM model. All data were analyzed using Microsoft Excel and PASW statistics 18.

\section{Results}

\section{Hunger and satiety}

The hungry versus satiated condition was confirmed by high ratings for hunger premeal $(71.5 \pm 3.8 \mathrm{~mm}$ VAS) and low ratings after the first meal (15.3 \pm 2.7 , difference $p<0.001)$ in both conditions.

\section{Energy intake and macronutrient composition of the meals}

Comparing the first meal and the second meal, energy intake was significantly lower during the second meal (Table $1, \mathrm{P}<0.001$ ). In the high protein condition, the macronutrient composition was significantly different compared with the $\mathrm{C}$ condition (Table 1, P<0.001). Fat intake was not significantly different comparing the conditions. During the second meal, the macronutrient composition of the meals was not significantly different comparing the $C$ with the $P$ condition (Table 1). Energy intake was not significantly different between the $C$ versus $P$ conditions. The reduction in energy intake implied a relative reduction of carbohydrate intake in the C condition from $69.9 \mathrm{En} \%$ to $43.0 \mathrm{En} \%$ and a relative reduction of protein in the $\mathrm{P}$ condition from $54.7 \mathrm{En} \%$ to $16.9 \mathrm{En} \%$, thereby showing a shift in macronutrient composition.

\section{Liking and wanting in the carbohydrate versus protein condition}

Behavioral liking and wanting ratings were lower after the first meal $(p<0.001)$, but the change in wanting was of a seven times higher magnitude (liking: $-0.09 \pm 0.14$, wanting: $-0.72 \pm 0.47$, liking vs. wanting $p<0.001$ ).

In the putamen, liking TRS was reduced post meal in the protein condition, but not in the carbohydrate condition (Figure 2A, interaction effect, protein < carbohydrates, $P<0.03)$. A similar trend was found for wanting TRS after the first meal in the hypothalamic region. (Figure $2 \mathrm{~B}$, interaction effect, protein< carbohydrates, $\mathrm{P}=0.067$ ).

When relating the change in energy intake from the first meal to the second meal, no significant relations between energy intake and wanting signaling were found. 
Conversely, the change in liking signaling was inversely related to the change in energy intake from pre-meal to post meal in the carbohydrate conditions, in the hypothalamic region and putamen (Figure $2 \mathrm{C}$ and $\mathrm{D}, \mathrm{P}=0.02$ and 0.08 ), while these parameters exhibited a positive correlation, in the protein conditions in the hypothalamic region (Figure $2 \mathrm{C}, \mathrm{P}=0.03$ ). These difference in brain signaling between the protein and carbohydrate condition was significant in both regions $(\mathrm{P}<0.01)$.

\section{Discussion}

High hunger ratings pre-meal and low hunger-ratings after the first meal and a significantly decreased energy intake during the second meal compared with the first meal, confirmed the hungry versus satiated state, while significantly different macronutrient composition confirmed the high $\mathrm{C}$ versus high $\mathrm{P}$ conditions during the first meal. During the second meal, the macronutrient composition was not different between the conditions, thus a condition-specific shift occurred from the first meal to the second meal. Behavioral liking and wanting was not different between the $C$ and $\mathrm{P}$ condition. Both decreased significantly from before to after the first meal, while confirming the higher relative stability of liking compared with wanting: the decrease in wanting was of a seven times higher magnitude. This is in line with expectations about liking and wanting as well as earlier findings that indicated strong decreases in behavioral wanting over a meal consumption while changes in liking were either absent or relatively small14,18,19,20. Previously, we have shown that liking and wanting are affected differently by different food items (cottage cheese versus chocolate mousse) that were liked significantly differently ${ }^{19}$. When the differences between the food items are limited to the macronutrient composition, such as in the present study, the subsequent changes in behavioral liking and wanting are not affected differently.

In the brain, liking TRS in the putamen showed an interaction effect of meal and condition, such that it was reduced after the first meal in the protein condition while it did not change in the carbohydrate condition. The decrease in energy intake, containing a relative decrease in protein intake from the first meal to the second meal, was positively related to the decrease in liking TRS in the protein conditions, while it was overall negatively related in carbohydrate conditions in the putamen and hypothalamic region. Additionally, liking TRS was higher in the protein condition compared with the carbohydrate condition, after the first meal compared with premeal in the putamen and this was underscored by a similar trend of wanting TRS in the hypothalamus. Thereby, liking TRS exhibited significant opposite directionality comparing the carbohydrate versus protein conditions, without leading to a differential behavioral effect in the short term. Additional, previous studies have shown both increased and decreased signaling associated with motivation to eat ${ }^{18,21,22,23,24}$.

In the present study, the different directionality of the liking TRS in the putamen and hypothalamic region may underscore the different changes of macronutrient intakes from the first meal to the second meal, both resulting in similar macronutrient intakes during the second meals. Therefore the liking TRS might represent the relative change in food choice at the following meal. 
A

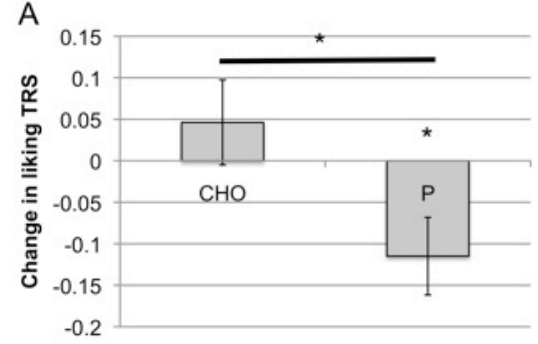

C

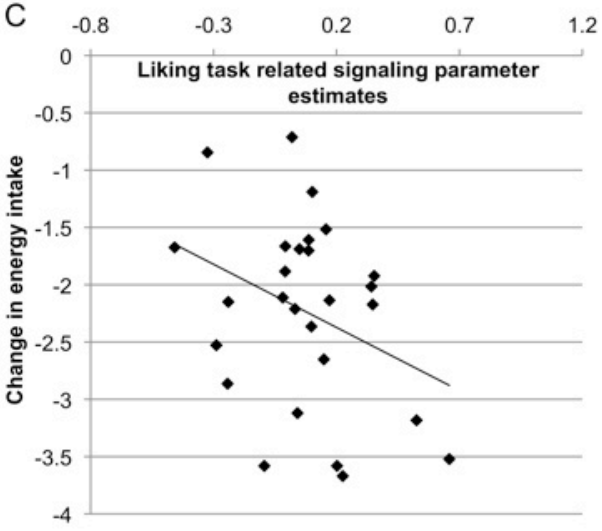

E

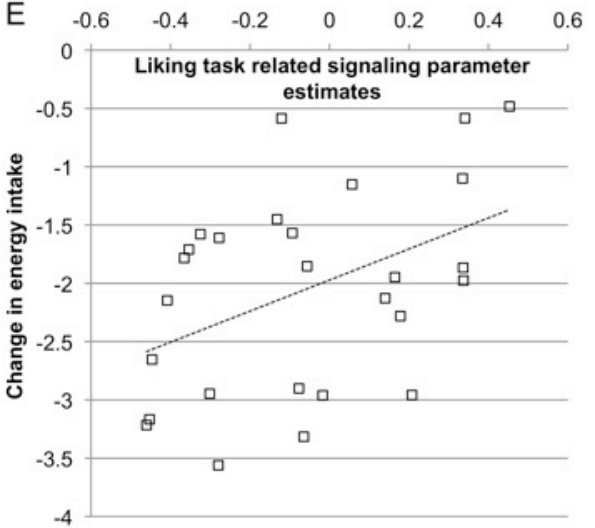

B
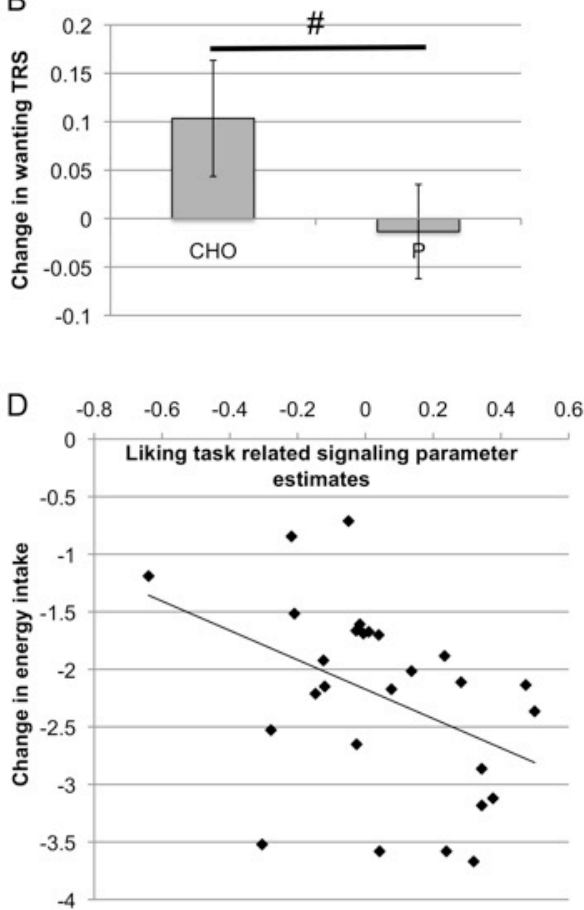

$\mathrm{F}$

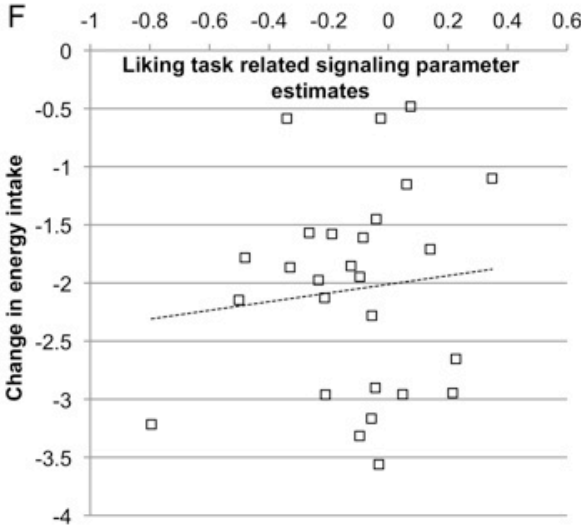

Figure 2: Functional data from the putamen and hypothalamic regions.

A: Change in liking TRS over the meal in the putamen over the meal, with significant interaction effect meal $x$ condition, $P=0.025$. $B$ : Change of wanting task related signaling (TRS) over the meal in the hypothalamic region, with a trend towards interaction effect meal $x$ condition, \# $P=0.067$. Data is represented as mean $\pm S E M(A$ and $B)$. The change in liking TRS related to the change in energy intake from breakfast to the second meal, in the $C$ condition in $C$ : the putamen $(P<0.04)$ and $D$ : the hypothalamic region $(P<0.09)$ and in the $P$ condition in $E$ : the putamen (n.s.) and $F$ : the hypothalamic region $(P<0.04)$.

In summary, the present study showed that after a carbohydrate rich meal, but not after a protein rich meal, wanting TRS was increased, which may potentially stimulate energy intake. Finally, the increase in liking TRS after a carbohydrate rich meal was inversely related to the decrease in energy intake. This underscored 
especially the relative decrease in carbohydrate intake. Similarly, the decrease in liking TRS after a protein rich meal positively related to the decrease in energy intake, underscoring the relative decrease in protein intake.

In conclusion, wanting TRS and liking TRS were affected differentially after carbohydrate or protein rich meals, underscoring subsequent energy intake and shift in macronutrient composition.

\section{References:}

Seidell, J. C. Epidemiology and health economics of obesity. Medicine 34, 506-509 (2006).

Catenacci, V. A., Hill, J. O. \& Wyatt, H. R. The Obesity Epidemic. Clinics in Chest Medicine 30, 415-444 (2009).

Westerterp, K. R. \& Speakman, J. R. Physical activity energy expenditure has not declined since the 1980s and matches energy expenditures of wild mammals. Int J Obes 32, 1256-1263 (2008).

4 Rutters, F., Nieuwenhuizen, A. G., Lemmens, S. G. T., Born, J. M. \& Westerterp-Plantenga, M. S. Acute Stressrelated Changes in Eating in the Absence of Hunger. Obesity 17, 72-72 (2009).

5 Epstein, L. H., Leddy, J. J., Temple, J. L. \& Faith, M. S. Food reinforcement and eating: a multilevel analysis. Psychological bulletin 133, 884-906 (2007).

6 Erlanson-Albertsson, C. How palatable food disrupts appetite regulation. Basic Clin Pharmacol Toxicol 97, 61-73 (2005)

7 Beaver, J. D. et al. Individual Differences in Reward Drive Predict Neural Responses to Images of Food. J. Neurosci. 26, 5160-5166, doi:10.1523/jneurosci.0350-06.2006 (2006).

8 Chambers, E. S., Bridge, M. W. \& Jones, D. A. Carbohydrate sensing in the human mouth: effects on exercise performance and brain activity. J Physiol 587, 1779-1794, doi:jphysiol.2008.164285 [pii] 10.1113/jphysiol. 2008.164285 (2009).

9 Drewnowski, A., Krahn, D. D., Demitrack, M. A., Nairn, K. \& Gosnell, B. A. Taste responses and preferences for sweet high-fat foods: evidence for opioid involvement. Physiol Behav 51, 371-379 (1992).

10 Melanson, K. J., Westerterp-Plantenga, M. S., Saris, W. H. M., Smith, F. J. \& Campfield, L. A. Blood glucose patterns and appetite in time-blinded humans: carbohydrate versus fat. Am J Physiol Regul Integr Comp Physiol 277, R337-345 (1999).

11 Soenen, S. \& Westerterp-Plantenga, M. S. Proteins and satiety: implications for weight management. Curr Opin Clin Nutr Metab Care 11, 747-751, doi:10.1097/MCO.0b013e328311a8c4 00075197-200811000-00012 [pii] (2008).

12 Veldhorst, M. et al. Protein-induced satiety: Effects and mechanisms of different proteins. Physiology \& Behavior 94, 300-307 (2008).

13 Berridge, K. C. Food reward: brain substrates of wanting and liking. Neurosci Biobehav Rev 20, 1-25 (1996).

14 Born, J. M., Lemmens, S. G. T., Formisano, E., Goebel, R. \& Westerterp-Plantenga, M. S. Differences between liking and wanting signals in the human brain and relations with BMl and cognitive dietary restraint. Am J Clin Nutr 94(2): 392-403 (2011).

15 Born, J. M., Lemmens, S. G. T., Formisano, E., Goebel, R. \& Westerterp-Plantenga, M. S. Differences between liking and wanting signals in the human brain and relations with cognitive dietary restraint and body mass index. Am J Clin Nutr 94, 392-403, doi:10.3945/ajcn.111.012161 (2011).

16 Harris, J. A. \& Benedict, F. G. A Biometric Study of Human Basal Metabolism. Proc Natl Acad Sci U S A 4, 370-373 (1918).

17 Baecke, J., Burema, J. \& Frijters, J. A short questionnaire for the measurement of habitual physical activity in epidemiological studies. The American Journal of Clinical Nutrition 36, 936-942 (1982).

18 Born, J. M. et al. Acute stress and food-related reward activation in the brain during food choice during eating in the absence of hunger. Int J Obes 34, 172-181 (2010).

19 Lemmens, S. G. T. et al. Eating what you like induces a stronger decrease of 'wanting' to eat. Physiology \& Behavior 98, 318-325, doi:10.1016/j.physbeh.2009.06.008 (2009).

20 Lemmens, S. G., Rutters, F., Born, J. M. \& Westerterp-Plantenga, M. S. Stress augments food 'wanting' and energy intake in visceral overweight subjects in the absence of hunger. Physiology \& Behavior 103(2): 157-163 (2010).

21 Davis, C. et al. From motivation to behaviour: a model of reward sensitivity, overeating, and food preferences in the risk profile for obesity. Appetite 48, 12-19 (2007).

22 Davis, C., Strachan, S. \& Berkson, M. Sensitivity to reward: implications for overeating and overweight. Appetite 42, 131-138, doi:10.1016/j.appet.2003.07.004 S0195666303001326 [pii] (2004).

23 Franken, I. H. \& Muris, P. Individual differences in reward sensitivity are related to food craving and relative body weight in healthy women. Appetite 45, 198-201, doi:S0195-6663(05)00042-5 [pii] 10.1016/j.appet.2005.04.004 (2005).

24 Wang, G. J. et al. Brain dopamine and obesity. Lancet 357, 354-357 (2001). 
Chapter 7

General Discussion 


\section{General discussion}

In the research presented in this thesis, the brain representation of the rewarding value of food, defined as liking and wanting was studied. Firstly, it is discussed, which regions are involved in respectively liking and wanting task related signaling (TRS). Secondly, the effects of other factors, namely dietary restraint, body weight, HPA-axis activation and shifts in macronutrient intakes on the representation of the rewarding value will be discussed.

\section{Overview of findings}

Behaviorally, liking and wanting represent two distinguishable concepts: Wanting was defined as the motivation to obtain a food item, i.e. a food item selection and some of the food item had to be eaten. In contrast, liking was defined as the hedonic preference rating of each food item. While both liking and wanting decreased over the test meals, the decrease in liking was of an approximately seven times smaller magnitude (Chapter 6). The brain activation associated with the liking and wanting tasks, was similar in the human brain compared with regions identified earlier in rodents, such that liking was signaled in homeostatic, taste related regions, while wanting was signaled in reward and motivation areas (Chapters 2-4). Regarding energy balance, it was shown that wanting task related signaling in the hypothalamus was related to energy intake and to body mass, in the hypothalamus and striatum. In normal weight subjects, this inverse relationship suggested reward deficiency. However, taking into account a weight range between a BMI of 19 to 33, this relationship was best described with a nonlinear model, suggesting a drive towards a BMI of 25 while normal weight, and reward dissociation in overweight subjects (Chapter 2 and 3). Moreover, the data in this thesis suggests that cognitive dietary restraint represents a tool to regulate food intake by modulating the change in signaling from the hungry to the satiated state in the nucleus accumbens (Chapter 2). This process then supports dietary restriction by limiting the rewarding value of food in the absence of hunger. This reduced rewarding value of food in the absence of hunger may offer a tool to oppose reward dissociation in overweight and obese subjects, or during stress. Reward dissociation at a central level was also indicated during stress i.e. higher cortisol levels, which lead to higher energy intakes, altered wanting task related signaling in the putamen, and disturbed the link between behavioral liking and wanting and the related brain signaling (Chapter 4 and 5). Importantly, effects of cognitive dietary restraint, i.e. reducing the rewarding value of food in the absence of hunger, opposed the effects seen with increased HPA-axis activation and those seen in overweight and obese subjects. Finally, shifts in macronutrient intakes were predicted by brain signaling with opposite directionality dependent on the macronutrient, i.e. protein vs. carbohydrate. This may represent a central modulation of motivational pathways that can be used to control food choice and energy intake, or which may lead to compensation for extreme macronutrient intakes (Chapter 6). 


\section{Liking and wanting in the brain}

Liking and wanting was signaled through partially divergent and partially convergent pathways in the human brain (chapter 2-4), which is in line with previous findings in the brains of rodents ${ }^{30,48,49,50,51,52,53}$ : liking TRS was found in the anterior insula, posterior insula, posterior cingulate, thalamus and visual areas, wanting TRS appeared in the striatum, i.e. caudate, putamen and globus pallidus, and additionally in the thalamus and visual areas. Thus liking appeared in regions associated with taste perception and hedonic properties of food, while wanting was related to regions that are involved in reward related signaling $30,33,48,49,50,51,52,53,54,56,61,134$, which may also explain why behavioral wanting was affected much more by satiety than liking (all chapters).

\section{Reward models}

Wanting TRS was observed in several regions involved in the homeostatic and reward pathways, namely the hypothalamus and striatum and this signaling was related to body mass (Chapter 2). Importantly, in the presented study including subjects with body mass ranging from low normal weight $(\mathrm{BMI}=19)$ to obese $(\mathrm{BMI}=$ 33), it was found that a linear model, such as either reward sensitivity or reward deficiency, does not explain the relation between body mass and wanting TRS (Chapter 3). Subsequently, it was shown that a nonlinear model approach explained the relation between body mass and wanting TRS in the absence of hunger significantly better, predominantly in the hypothalamic region, yet to a lesser degree also in the striatum and nucleus accumbens. Thus wanting TRS was lowest in subjects with a BMI of approximately 25 , and progressively higher with BMIs lower and higher than 25. The involvement of the hypothalamus suggests, that the homeostatic system exerts significant influence on wanting related processes. Simultaneously, the hypothalamic deregulation associated with higher body weights suggests that the reward system may significantly affect the hypothalamic signaling as well: previous studies suggest that reward dissociation, possibly reduced dopaminergic signaling ${ }^{76,135}$, may underlie hypothalamic deregulation (Chapter 2 and 3). The data presented here, consistently suggests a bias toward a BMI of approximately 25 in normal weight subjects: The lower the BMI, the higher wanting TRS in the striatum and nucleus accumbens in the satiated condition, implying support for eating in the absence of hunger. Studies on morbidity and mortality generally show a similar characteristic: Related to body mass, morbidity and mortality consistently shows a u-shaped curve with nadir values around a BMI of $25-26^{136,137,138}$. Thus a bias toward a high normal weight or slightly overweight BMI seems biologically favorable. Conversely in overweight subjects (BMI >25), increasing body mass was associated with progressively higher wanting TRS. The findings in overweight and obese subjects indicate reward dissociation, i.e. loss of the closely balanced link between reward pathways and homeostatic signaling. This reward dissociation implies that signaling of the rewarding value of food is not appropriate: An increased motivation to eat in the absence of hunger in subjects with overweight or obesity, may be detrimental for their health in the long term, as it likely leads to maintenance or exacerbation of excessive body weight and consequently co-morbities such as the metabolic syndrome ${ }^{4,139}$ and a higher mortality ${ }^{136,137,138}$. 
Biologically, evidence for reward dissociation has been given in previous studies: Dopamine D2 receptor availability in reward related areas of the brain, such as the ventral striatum, decreased with increasing body mass in morbidly obese subjects. In addition, genetic variation for the dopamine D2 receptor to brain signaling in reward related areas of the striatum was predictive for future body weight gain, indicating significant contribution of genetic predisposition in the development of obesity ${ }^{66,76,140}$. Furthermore, deregulation of multiple peripheral hormones has been reported, including increased leptin and insulin resistance, as well as disturbed glucose and fatty acid levels ${ }^{4,141,142}$. The increased hormone levels, may then significantly affect the homeostatic circuits of the arcuate nucleus, lateral hypothalamus and PVN. Ultimately, the disturbed activity in these regions can affect downstream signaling throughout the circuitry of food intake regulation and directly contributing to disturbed central signaling via receptors in the hypothalamus and ultimately reward dissociation ${ }^{15,18,22,24,25}$. Taken together, while in normal weight subjects there were indications for reward deficiency; however, a linear relationship could not be established consistently over the normal weight to obese range (BMI = 19-33). Instead the nonlinear model fit suggested that above a BMI of 25, wanting TRS may be deregulated. This deviation from linear reward models may be described as reward dissociation, which may ultimately bias the subjects to higher energy intake and maintenance or exacerbation of overweight and obesity.

\section{The rewarding value of food related to energy balance}

Related to energy balance, it was confirmed that liking and wanting diverge on key aspects of energy balance regulation: wanting task related signaling (TRS) in regions associated with reward, such as the striatum (both ventral and dorsal) was related to body mass $30,48,49,50,51,52,53$. Moreover, in the hypothalamus, wanting TRS was related to body mass, as well as hunger and satiety, and energy intake, indicating that wanting TRS was connected to the non-homeostatic reward circuits as well as homeostatic systems in the hypothalamus ${ }^{7,15,16}$. In contrast, liking TRS was predominantly found in regions associated with taste and hedonic properties of food, consistently in the anterior insulae ${ }^{61,134}$ (chapter 2 and 3). Overlapping areas for liking and wanting TRS were seen in the thalamus, visual cortex, the orbitofrontal cortex (OFC) and the nucleus accumbens (NAc), and these results are in line with expectations: except olfaction, taste and sensory signals from the mouth and from the gastrointestinal tract are projected through the nucleus of the solitary tract (NTS) to the thalamus, from which these signals are projected to the taste cortex and higher centers such as the prefrontal cortex ${ }^{18,143}$. At the same time, the thalamus is involved in the signaling from the ventral tegmental area (VTA) to the amygdala, NAc, hypothalamus, prefrontal cortex (PFC) and the sensory-motor cortex $32,33,34$. Therefore, the overlap observed for liking and wanting in this region could either represent parallel pathways, or it may imply low level modulation between the homeostatic and non-homeostatic motivational pathways. In the studies presented here, liking TRS in the hypothalamic region was observed only related to shifts in macronutrient intakes (Chapter 6), while wanting TRS was observed in this region and the level of this signaling when fasted, predicted energy intake during breakfast (Chapter 2). The involvement of the hypothalamus in signaling of both liking and 
wanting, may be explained by earlier findings: It was previously shown that the hypothalamus links to both homeostatic and motivational systems, underlining its pivotal role in homeostatic, yet also non-homeostatic energy intake $15,18,22,24,25,31,33$.

\section{Cognitive dietary restraint to oppose reward dissociation}

In achieving energy balance, energy intake has to be regulated. This regulation depends partly on the rewarding value of food, such that highly palatable food in some cases may lead to excessive energy intake ${ }^{16}$. To counter this drive towards overeating, cognitive dietary restraint may represent a behavioral tool by which energy intake is controlled $41,69,103,144,145$. While it has been shown how cognitive dietary restraint together with disinhibition and feelings of hunger may affect food intake $^{145}$, it was previously unknown how these processes may be reflected in the brain. In this thesis, it is shown that cognitive dietary restraint may be linked to liking TRS in the nucleus accumbens (Chapter 2). In this region, dietary restraint scores predicted the change of liking TRS from pre-meal to post-meal, such that a high dietary restraint score was related to high pre-meal liking TRS and relatively low post-meal TRS. From the nucleus accumbens, this modulation may affect the homeostatic pathways through projections to the orbitofrontal cortex and the hypothalamus, and the reward system through projections to the striatum. Thereby, dietary restraint seems to decrease the rewarding value of food in the absence of hunger ${ }^{18,31,33}$. Comparing the effects of body weight on brain signaling with the effects of cognitive dietary restraint on brain signaling, they appear to result in opposing effects in overweight and obese subjects: increasing body mass above a BMI of 25 lead to increased signaling in the hypothalamus and striatum, high cognitive dietary restraint led to decreased post-meal signaling in the nucleus accumbens. Thus cognitive dietary restraint may be a tool to reduce reward dissociation. Taken together, cognitive dietary restraint reduces the rewarding value of food in the absence of hunger, thereby assisting in dietary restriction and possibly additionally reducing reward dissociation in overweight subjects.

\section{The effect of the hypothalamus pituitary (HPA) axis on the rewarding value of food}

The conducted studies presented here, link stress related changes that occur in central signaling to behavioral scores and energy intake: During stress, higher levels of cortisol were measured, confirming acute HPA-axis activation; simultaneously, food choice related brain signaling in the putamen was decreased, coinciding with an increased choice for items that were crispy and had a high fullness of taste. This altered food choice then resulted in slightly increased energy intake in the absence of hunger (Chapter 4). These results are in line with previous studies that showed increased eating in the absence of hunger of snack foods ${ }^{44}$. Additionally, the disruptive effects of HPA-axis activation were found on the connection between behavioral liking and wanting with the task related brain signaling: In the fasted state and with low activation of the HPA-axis (low cortisol levels), behavioral liking was related to liking TRS in the anterior insula and behavioral wanting was related to 
wanting TRS in the anterior insula, nucleus accumbens and thalamus. All of these relationships were lost in a relatively high HPA-activated condition, while two new relationships appeared in the satiated state, i.e. behavioral liking was related with liking TRS in the nucleus accumbens and behavioral wanting with wanting TRS in the caudate (Chapter 5). Together, the above findings suggest that stress and increased HPA-axis activation act to bias the brain towards higher energy intake via reward dissociation: Firstly, the overall activation during high HPA-activation was decreased; in the studies presented in this thesis, lower brain activation in specifically in reward related areas consistently predicted eating in the absence of hunger and higher energy intake during the meal following the respective scan (Chapter 2-5). Secondly, the link between behavioral liking and wanting and liking and wanting TRS in the regions that were normally associated was disrupted, dissociating the behavioral process from the appropriate brain signaling. Possibly, liking and wanting increased their effects in other, overall more reward related areas, i.e. the nucleus accumbens and caudate. Involving these motivational and reward areas at an inappropriate time, i.e. in the absence of hunger, may lead to changed signaling and ultimately to increased food intake. The effects of HPA-axis activation may be derived from multiple stages including the hormones corticotropin releasing hormone $(\mathrm{CRH})$, adreno-corticotrope hormone $(\mathrm{ACTH})$, or cortisol, each of which have been shown to act on the amygdala and paraventricular nucleus (PVN) of the hypothalamus exerting negative feedback for HPA-axis activation ${ }^{146}$. CRH signals to the amygdala and $\mathrm{PVN}^{147}$. From this nucleus, projections to the nucleus of the solitary tract (NTS), ventral tegmental area (VTA) and ultimately the striatum and nucleus accumbens ${ }^{18,32,33,34}$, may be the underlying processes that interfere with homeostatic and motivational signaling. Taken together, the presented studies concur with earlier speculations as well as indications that stress (and the associated HPA-axis activation) may play an important role in the etiology of the obesity epidemic ${ }^{103,109,120,148,149}$. Stress was associated with changes in food choice toward more crispiness and fullness of taste, lead to higher energy intake, and it disrupted the link between behavioral liking and wanting and the liking and wanting TRS in the brain. Thus, it is indicated that stress and increased HPA-axis activation may deregulate motivational, reward and homeostatic circuits, and deregulate the connection between wanting TRS and behavioral wanting. Thereby, HPA-axis activation may contribute to and exacerbate reward dissociation seen in overweight and obese subjects, and consequently contribute to changed food choice and higher energy intake.

\section{The effects of shifts in macronutrient composition on the rewarding value of food}

In this thesis, it was shown that a normal protein normal carbohydrate meal was consumed after a high protein breakfast, and this shift in macronutrient composition was related to the change in liking TRS in the putamen. Similarly, after high carbohydrate low protein intake during breakfast the following meal that was consumed had a normal macronutrient composition and this change in carbohydrate content of the meals was inversely related to wanting TRS in the hypothalamic region (Chapter 6). These findings suggest that shifts in macronutrient intakes, also 
those involved in compensation for extreme macronutrient intakes ${ }^{105,107}$, are supported by marked differences in brain signaling. Compensational behavior may be an evolutionary protective mechanism, to avoid specific nutrient deficiencies that can arise from lack of diversity in food intake. The related brain signaling may help to explain the reported poor adherence to extreme macronutrient intakes, which is seen in protein rich diets ${ }^{104,105,106,150:}$ : extreme macronutrient intakes appear to alter homeostatic brain signaling in the hypothalamus in a macronutrient specific direction. Additionally, they affected the putamen, which is involved in reward related processes ${ }^{13,31,32,33,34}$. Surprisingly, the change in liking TRS was differentially related to different macronutrient shifts in those two regions: the shift from high to low protein intake was related to liking TRS in the putamen, while high to low carbohydrate intake was inversely related to liking TRS in the hypothalamic region, suggesting that macronutrient specific effects may be exerted in a similar way to dietary restraint, i.e. affecting liking rather than wanting TRS in motivational circuits (Chapter 2). Together, it is suggested that macronutrient intake may be used as a nutritional tool to modulate central motivational regions and possibly thereby influence energy intake and food choice. Regarding the regions that were indicated, the hypothalamus receives macronutrient specific inputs through direct nutrient sensing receptors for glucose and amino acids ${ }^{15,18,22,24,25}$, as well as from the vagus nerve through the nucleus of the solitary tract (NTS) $20,21,22$. In contrast, macronutrient related signals arrive in the putamen through other regions, such as the hypothalamus and the NTS through the thalamus, as well as more complex pathways including multiple intermediate regions ${ }^{32,33,34}$. The position in the signaling route may explain why the shifts in liking TRS in the hypothalamus related more strongly to the shifts in macronutrient intakes, compared with the putamen. The involvement of the putamen indicates how macronutrient specific processes may affect motivational systems, ultimately resulting in macronutrient compensation as has been reported previously ${ }^{107}$. Taken together, extreme macronutrient intakes and the subsequent shifts to normal macronutrient content meals resulted in differential shifts in brain signaling in motivational and homeostatic regions that was dependent on the macronutrient involved. This differential signaling may bias the brain towards macronutrient compensation, while it may also indicate how extreme macronutrient intakes may assist as a nutritional tool to control energy intake.

\section{Conclusion}

Wanting TRS is consistent with motivation, reward and homeostatic signaling, and this signaling was associated with hunger and energy intake. Liking TRS appeared independently in hedonic regions. In the brain, liking TRS was involved in the modulation of the rewarding value of food due to cognitive dietary restraint and shifts in macronutrient intakes. In multiple studies, indications were found for reward dissociation, which is a disruption of motivational, reward and homeostatic signaling. Reward dissociation is represented by changed wanting TRS related to BMI, disrupted links between behavioral liking and wanting and the associated TRS. This reward dissociation was observed in overweight and obese subjects, as well as during high HPA-axis activation. In conclusion, homeostatic food intake regulation is represented by closely regulated liking and wanting signaling in the brain, which is 
related to behavioral liking and wanting, BMI and hunger. Overweight, obesity and stress resulted in reward dissociation, supporting excessive food intake and altered food choice, yet these effects may be reduced using cognitive dietary restraint as a tool.

\section{Future research perspectives}

In the studies presented in this thesis, liking and wanting related signaling pathways in the brain were assessed. Novel high field fMRI with field strengths of 7 Tesla and above, may offer the opportunity to study the networks that are involved in food intake regulation with a higher resolution and thereby in much more detail ${ }^{151}$. Thus in contrast to the current 3 Tesla system that was unable to discriminate sub-regions of the NAc or the hypothalamus, in future studies it may be feasible to study BOLD responses from these small functional sub-regions. While the use of functional MRI allows for exciting new insights, the underlying mechanisms are suggested to be studied in greater detail using other techniques: The use of specific neurotransmitter receptor agonists or antagonists in humans seems to be a promising approach, although it has been proven difficult in the past. It should be taken into account that undesired side effects of the drug, such as nausea or headaches may influence eating behavior, masking any effect for functional MRI. In addition, the pharmacokinetics of any psychoactive compound, may not be fully known, leading to unexpected results from side actions of the drug ${ }^{152}$. These effects have to be controlled for, by additional behavioral testing. To overcome these limitations, invasive techniques, such as micro-dialysis in rodent brains, may be used to assess neurotransmitter release directly ${ }^{153}$. Using this method, the neurotransmitter involved in reward can be assessed and related to overweight and obesity models. To specify this neurotransmitter involvement in humans, non invasive techniques, such as positron emission tomography, will be useful: New radio-ligands for use in PET imaging are currently being developed, making future receptor specific imaging of liking and wanting related processes feasible for dopamine, gamma amino butyric acid (GABA), endocannabinoid, serotonin, and other neurotransmitter signaling pathways ${ }^{154,155,156,157,158,159,160,161,162}$. This is especially relevant since even in overlapping regions for liking and wanting, separate neurotransmitters may be involved, and the pathways involved may indicate in greater detail of how liking and wanting diverge and converge. However, two factors limit the usefulness of PET as a research tool: Firstly, the use of radio-nucleotides implies that the number of scans that are allowable per subject is relatively small in order to limit radioactive exposure. Secondly, radio-nucleotides used have to be freshly prepared in a cyclotron as the isotopes used have very short half-lives, again to limit radioactive exposure ${ }^{163}$.

Regarding the relation between $\mathrm{BMI}$ and wanting TRS, it was shown that nonlinear modeling may be more suitable to explain the between subjects variability wanting TRS and BMI. Furthermore, it was previously shown that the genetic background may be predictive of reward and motivational signaling in humans ${ }^{66,77}$. Therefore, it is relevant to further study the role of the genetic background in these relationships between signaling, body mass, hunger and energy intake. 
Furthermore, in this thesis, effects of HPA-axis activation and body mass on wanting TRS are described. Previous reports indicated that stress levels are rising in the developed world and effects of stress have been especially observed in viscerally overweight subjects ${ }^{85}$. These subjects exhibit the highest incidence of the metabolic syndrome and it would be highly relevant to assess how stress affects the deregulation of wanting TRS, as observed in overweight subjects $5,82,149$. It was shown that activation of the HPA-axis, assessed by serum cortisol measurements, was associated with deregulation of wanting TRS alone, and in relation to behavioral wanting. So far, the stress response was studied on one level of the HPA axis, i.e. the measurement of cortisol. However, the deregulation that was indicated may occur at several levels op the HPA-axis, including CRH, ACTH and cortisol signaling, and may interact with the dopamine reward system ${ }^{92,93,94,95,96}$. Finally, interactions between the HPA-axis and the hypothalamus pituitary thyroid axis (HPT-axis) have been described ${ }^{164}$. Given that thyroid hormones regulate body temperature and metabolism and possibly food intake ${ }^{165,166}$, it is relevant to study how these thyroid hormones may be affected by HPA-axis activation, and how these hormones themselves may modulate signaling of the rewarding value of food. Overall, the relation between stress, food reward and food intake seems the most relevant issue for future research: Levels of stress are increasing together with indices for overweight and obesity $81,82,85,120$. Thorough understanding of how stress affects brain signaling and interferes with the HPA/HPT-axis and food choice may lead to the identification of foods that may help to manage these interactions. This management may include specifically altering food choice, it may indicate pharmacological targets in extreme cases, or identify characteristics of novel functional foods. 


\section{References}

1. Abler, B., Walter, H., Erk, S., Kammerer, H. \& Spitzer, M. Prediction error as a linear function of reward probability is coded in human nucleus accumbens. Neuroimage 31, 790 - 795 (2006).

2. Berridge, K.C. Food reward: brain substrates of wanting and liking. Neurosci Biobehav Rev 20, 1-25 (1996).

3. Born, J.M., et al. Acute stress and food-related reward activation in the brain during food choice during eating in the absence of hunger. Int J Obes 34, 172-181 (2010).

4. Bratcher, N.A., Farmer-Dougan, V., Dougan, J.D., Heidenreich, B.A. \& Garris, P.A. The role of dopamine in reinforcement: changes in reinforcement sensitivity induced by D1-type, D2-type, and nonselective dopamine receptor agonists. J Exp Anal Behav 84, 371-399 (2005).

5. McCabe, C., Cowen, P. \& Harmer, C. Neural representation of reward in recovered depressed patients. Psychopharmacology 205, 667-677 (2009).

6. Murray, E.A. The amygdala, reward and emotion. Trends in cognitive sciences 11, $489-497$ (2007).

7. Evans, A.H., et al. Compulsive drug use linked to sensitized ventral striatal dopamine transmission. Ann Neurol 59, 852-858 (2006).

8. Kelley, A.E., Baldo, B.A., Pratt, W.E. \& Will, M.J. Corticostriatal-hypothalamic circuitry and food motivation: Integration of energy, action and reward. Physiology \& Behavior 86, 773-795 (2005).

9. Gottfried, J.A., O'Doherty, J. \& Dolan, R.J. Encoding predictive reward value in human amygdala and orbitofrontal cortex. Science 301, 1104-1107 (2003).

10. Small, D.M., Jones-Gotman, M. \& Dagher, A. Feeding-induced dopamine release in dorsal striatum correlates with meal pleasantness ratings in healthy human volunteers. Neuroimage 19, 1709-1715 (2003).

11. Preuschoff, K., Quartz, S. \& Bossaerts, P. Human insula activation reflects risk prediction errors as well as risk. $J$ Neurosci 28, 2745 - 2752 (2008).

12. Rolls, E.T. Sensory processing in the brain related to the control of food intake. Proc Nutr Soc 66, $96-112$ (2007).

13. Volkow, N.D., et al. Low dopamine striatal D2 receptors are associated with prefrontal metabolism in obese subjects: possible contributing factors. Neuroimage 42, 1537-1543 (2008).

14. Wang, G.J., et al. Brain dopamine and obesity. Lancet 357, 354-357 (2001).

15. Allison, D.B., Faith, M.S., Heo, M. \& Kotler, D.P. Hypothesis Concerning the U-shaped Relation between Body Mass Index and Mortality. American Joumal of Epidemiology 146, 339-349 (1997).

16. Orpana, H.M., et al. BMI and Mortality: Results From a National Longitudinal Study of Canadian Adults. Obesity 18, 214-218 (2009).

17. Seidell, J.C., Visscher, T.L. \& Hoogeveen, R.T. Overweight and obesity in the mortality rate data: current evidence and research issues. Med Sci Sports Exerc 31, S597-601 (1999).

18. Despres, J.P. \& Lemieux, I. Abdominal obesity and metabolic syndrome. Nature 444, 881-887 (2006).

19. Eckel, R.H., Grundy, S.M. \& Zimmet, P.Z. The metabolic syndrome. The Lancet 365, 1415-1428 (2005)

20. Stice, E. \& Dagher, A. Genetic variation in dopaminergic reward in humans. Forum Nutr 63, 176-185 (2010).

21. Stice, E., Spoor, S., Bohon, C. \& Small, D.M. Relation Between Obesity and Blunted Striatal Response to Food Is Moderated by TaqIAA1 Allele. Science 322, 449-452 (2008).

22. Douyon, L. \& Schteingart, D.E. Effect of obesity and starvation on thyroid hormone, growth hormone, and cortisol secretion. Endocrinology and metabolism clinics of North America 31, 173-189 (2002).

23. Unger, R.H. Lipid overload and overflow: metabolic trauma and the metabolic syndrome. Trends Endocrinol Metab 14, 398-403 (2003).

24. Blevins, J.E. \& Baskin, D.G. Hypothalamic-brainstem circuits controlling eating. Forum Nutr 63, $133-140$ (2010).

25. Langhans, W. \& Geary, N. Overview of the physiological control of eating. Forum Nutr 63, 9-53 (2010).

26. Moran, T.H. Hypothalamic nutrient sensing and energy balance. Forum Nutr 63, 94-101 (2010).

27. Munzberg, H. Leptin-signaling pathways and leptin resistance. Forum Nutr 63, 123-132 (2010).

28. Berthoud, H.-R. Multiple neural systems controlling food intake and body weight. Neuroscience \& Biobehavioral Reviews 26, 393-428 (2002).

29. Erlanson-Albertsson, C. How palatable food disrupts appetite regulation. Basic Clin Pharmacol Toxicol 97, 61-73 (2005).

30. Berthoud, H.R. Neural control of appetite: cross-talk between homeostatic and non-homeostatic systems. Appetite 43, 315-317 (2004).

31. Chromiak, J.A. \& Antonio, J. Use of amino acids as growth hormone-releasing agents by athletes. Nutrition 18 , 657-661 (2002).

32. Balleine, B.W., Delgado, M.R. \& Hikosaka, O. The Role of the Dorsal Striatum in Reward and Decision-Making. J. Neurosci. 27, 8161-8165 (2007).

33. Sánchez-Lasheras, C., Christine Könner, A. \& Brüning, J.C. Integrative neurobiology of energy homeostasisneurocircuits, signals and mediators. Frontiers in Neuroendocrinology 31, 4-15 (2010).

34. Berthoud, H.R. Homeostatic and non-homeostatic pathways involved in the control of food intake and energy balance. Obesity (Silver Spring) 14 Suppl 5, 197S-200S (2006).

35. Lejeune, M.P., Van Aggel-Leijssen, D.P., Van Baak, M.A. \& Westerterp-Plantenga, M.S. Effects of dietary restraint vs exercise during weight maintenance in obese men. Eur J Clin Nutr 57, 1338-1344 (2003).

36. Lemmens, S.G., et al. Dietary Restraint and Control Over 'Wanting'Following Consumption of 'Forbidden' Food. Obesity 18, 1926-1931 (2010). 
37. Stunkard, A.J. \& Messick, S. The three-factor eating questionnaire to measure dietary restraint, disinhibition and hunger. J Psychosom Res 29, 71-83 (1985).

38. Vogels, N., Diepvens, K. \& Westerterp-Plantenga, M.S. Predictors of long-term weight maintenance. Obes Res 13, 2162-2168 (2005).

39. Wardle, J., Steptoe, A., Oliver, G. \& Lipsey, Z. Stress, dietary restraint and food intake. J Psychosom Res 48, 195-202 (2000).

40. Rutters, F., Nieuwenhuizen, A.G., Lemmens, S.G.T., Born, J.M. \& Westerterp-Plantenga, M.S. Acute Stressrelated Changes in Eating in the Absence of Hunger. Obesity 17, 72-72 (2009).

41. Makino, S., Hashimoto, K. \& Gold, P.W. Multiple feedback mechanisms activating corticotropin-releasing hormone system in the brain during stress. Pharmacol.Biochem.Behav. 73, 147-158 (2002).

42. Dallman, M.F., Pecoraro, N.C. \& la Fleur, S.E. Chronic stress and comfort foods: self-medication and abdominal obesity. Brain Behav Immun 19, 275-280 (2005).

43. Adam, T.C. \& Epel, E.S. Stress, eating and the reward system. Physiology \& Behavior 91, $449-458$ (2007).

44. Zellner, D.A., et al. Food selection changes under stress. Physiol Behav 87, 789-793 (2006).

45. Rutters, F., Nieuwenhuizen, A.G., Lemmens, S.G.T., Born, J.M. \& Westerterp-Plantenga, M.S. HypothalamicPituitary-Adrenal (HPA) axis functioning in relation to body fat distribution. Clinical Endocrinology 72, 738-743 (2010).

46. Pecoraro, N., Reyes, F., Gomez, F., Bhargava, A. \& Dallman, M.F. Chronic stress promotes palatable feeding, which reduces signs of stress: feedforward and feedback effects of chronic stress. Endocrinology 145, 3754-3762 (2004).

47. Soenen, S. \& Westerterp-Plantenga, M.S. Proteins and satiety: implications for weight management. Curr Opin Clin Nutr Metab Care 11, 747-751 (2008).

48. Westerterp-Plantenga, M.S., MJ, I.J. \& Wijckmans-Duijsens, N.E. The role of macronutrient selection in determining patterns of food intake in obese and non-obese women. Eur J Clin Nutr 50, 580-591 (1996).

49. Veldhorst, M., et al. Protein-induced satiety: Effects and mechanisms of different proteins. Physiology \& Behavior 94, 300-307 (2008).

50. Lejeune, M.P., Westerterp, K.R., Adam, T.C., Luscombe-Marsh, N.D. \& Westerterp-Plantenga, M.S. Ghrelin and glucagon-like peptide 1 concentrations, 24-h satiety, and energy and substrate metabolism during a high-protein diet and measured in a respiration chamber. Am J Clin Nutr 83, 89-94 (2006).

51. Veldhorst, M.A., Westerterp, K.R., van Vught, A.J. \& Westerterp-Plantenga, M.S. Presence or absence of carbohydrates and the proportion of fat in a high-protein diet affect appetite suppression but not energy expenditure in normal-weight human subjects fed in energy balance. Br J Nutr 104, 1395-1405 (2010).

52. Berthoud, H.R. Mind versus metabolism in the control of food intake and energy balance. Physiol Behav 81, 781-793 (2004).

53. Schwartz, G.J. Brainstem integrative function in the central nervous system control of food intake. Forum Nutr 63, 141-151 (2010).

54. Zheng, H., Lenard, N.R., Shin, A.C. \& Berthoud, H.R. Appetite control and energy balance regulation in the modern world: reward-driven brain overrides repletion signals. Int J Obes (Lond) 33 Suppl 2, S8-13 (2009).

55. De Martino, F., et al. Whole brain high-resolution functional imaging at ultra high magnetic fields: An application to the analysis of resting state networks. Neuroimage 57, 1031-1044 (2011).

56. Kvernmo, T., Hartter, S. \& Burger, E. A review of the receptor-binding and pharmacokinetic properties of dopamine agonists. Clin Ther 28, 1065-1078 (2006).

57. Hoebel, B.G., Hernandez, L., Schwartz, D.H., Mark, G.P. \& Hunter, G.A. Microdialysis Studies of Brain Norepinephrine, Serotonin, and Dopamine Release During Ingestive Behavior Theoretical and Clinical Implicationsa. Annals of the New York Academy of Sciences 575, 171-193 (1989).

58. Cliffe, I.A. A retrospect on the discovery of WAY-100635 and the prospect for improved 5-HT(1A) receptor PET radioligands. Nucl Med Biol 27, 441-447 (2000).

59. Henriksen, G. \& Willoch, F. Imaging of opioid receptors in the central nervous system. Brain 131, 1171-1196 (2008).

60. Henry, T.R. Functional neuroimaging with positron emission tomography. Epilepsia 37, 1141-1154 (1996).

61. Zhang, M.R. \& Suzuki, K. [18F]Fluoroalkyl agents: synthesis, reactivity and application for development of PET ligands in molecular imaging. Curr Top Med Chem 7, 1817-1828 (2007).

62. van der Goot, H. \& Timmerman, H. Selective ligands as tools to study histamine receptors. Eur J Med Chem $\mathbf{3 5}$, 5-20 (2000).

63. Evens, N. \& Bormans, G.M. Non-invasive imaging of the type 2 cannabinoid receptor, focus on positron emission tomography. Curr Top Med Chem 10, 1527-1543 (2010).

64. Horti, A.G. \& Van Laere, K. Development of radioligands for in vivo imaging of type 1 cannabinoid receptors (CB1) in human brain. Curr Pharm Des 14, 3363-3383 (2008).

65. Katsifis, A. \& Kassiou, M. Development of radioligands for in vivo imaging of GABA(A)-benzodiazepine receptors. Mini Rev Med Chem 4, 909-921 (2004).

66. Maziere, B., Coenen, H.H., Halldin, C., Nagren, K. \& Pike, V.W. PET radioligands for dopamine receptors and reuptake sites: chemistry and biochemistry. Int J Rad Appl Instrum B 19, 497-512 (1992).

67. Devine, C.E. \& Mawlawi, O. Radiation Safety With Positron Emission Tomography and Computed Tomography. Seminars in Ultrasound, CT, and MRI 31, 39-45 (2010). 
68. Stice, E., Yokum, S., Bohon, C., Marti, N. \& Smolen, A. Reward circuitry responsivity to food predicts future increases in body mass: Moderating effects of DRD2 and DRD4. Neuroimage 50, 1618-1625 (2010).

69. Bjorntorp, P. \& Rosmond, R. Obesity and cortisol. Nutrition 16, 924-936 (2000).

70. Hutley, L. \& Prins, J.B. Fat as an endocrine organ: relationship to the metabolic syndrome. Am J Med Sci 330, 280-289 (2005).

71. Kyrou, I., Chrousos, G.P. \& Tsigos, C. Stress, visceral obesity, and metabolic complications. Annals of the New York Academy of Sciences 1083, 77-110 (2006).

72. Fulton, S., Richard, D., Woodside, B. \& Shizgal, P. Interaction of $\mathrm{CRH}$ and energy balance in the modulation of brain stimulation reward. Behav Neurosci 116, 651-659 (2002).

73. Pani, L., Porcella, A. \& Gessa, G.L. The role of stress in the pathophysiology of the dopaminergic system. Mol Psychiatry 5, 14-21 (2000).

74. Pruessner, J.C., Champagne, F., Meaney, M.J. \& Dagher, A. Dopamine release in response to a psychological stress in humans and its relationship to early life matemal care: a positron emission tomography study using [11C] raclopride. J Neurosci 24, 2825-2831 (2004).

75. Salamone, J.D., Cousins, M.S. \& Snyder, B.J. Behavioral functions of nucleus accumbens dopamine: empirical and conceptual problems with the anhedonia hypothesis. Neurosci Biobehav Rev 21, 341-359 (1997).

76. Czyrak, A., Mackowiak, M., Chocyk, A., Fijal, K. \& Wedzony, K. Role of glucocorticoids in the regulation of dopaminergic neurotransmission. Pol J Pharmacol 55, 667-674 (2003).

77. Helmreich, D.L., Parfitt, D.B., Lu, X.Y., Akil, H. \& Watson, S.J. Relation between the hypothalamic-pituitary-thyroid (HPT) axis and the hypothalamic-pituitary-adrenal (HPA) axis during repeated stress. Neuroendocrinology $\mathbf{8 1}$ 183-192 (2005).

78. Ismail-Beigi, F. \& Edelman, I.S. The Mechanism of the Calorigenic Action of Thyroid Hormone: Stimulation of Na+ + K+-activated adenosinetriphosphatase activity. The Journal of General Physiology 57, 710-722 (1971)

79. Silva, J.E. The thermogenic effect of thyroid hormone and its clinical implications. Ann.Intern.Med. 139, 205-213 (2003).

80. Epel, E.S., et al. Stress and body shape: stress-induced cortisol secretion is consistently greater among women with central fat. Psychosomatic medicine 62, 623-632 (2000). 
Summary 
The etiology of overweight and obesity has been attributed to numerous causes, including genetic, physiological, environmental and behavioral factors. Regarding energy balance, the focus has been mostly on satiety, both behaviorally and hormonally; however, this may be insufficient: There are indications that reward balance may be an essential factor contributing to energy intake, without necessarily leading to energy balance.

In this thesis, methods are explored to model brain activity using functional magnetic resonance imaging (fMRI) and the brain representation of the rewarding value of food. This rewarding value is defined as the combination of 'liking' and 'wanting' of food. Liking is the hedonic preference for a food item. Conversely, wanting is the motivation to obtain and consume a food item. While liking and wanting are related, they are not the same process: At times, even highly liked food items are not wanted. In the present studies, it was found that both liking and wanting decreased over the test meals. However, the decrease in liking was of an approximately seven times smaller magnitude (Chapter 6). This indicates that liking was relatively unaffected by hunger and satiety, compared with wanting, which decreased very significantly. In the human brain, regions that were involved during the liking and wanting tasks, were similar to regions identified earlier in rodents: Specifically, liking was signaled in homeostatic, taste related regions: the anterior insula, posterior insula, posterior cingulate. Wanting was signaled in reward and motivation related areas: the caudate, putamen and globus pallidus. In the thalamus and visual areas both liking and wanting were represented (Chapters 2-4). Thereby, liking and wanting were found in mostly divergent pathways, that converged in specific regions, such as the thalamus. Furthermore, it was shown that wanting task related signaling in the hypothalamus was related to energy intake, and in addition, it was related to body mass in the hypothalamus and striatum. In normal weight subjects, this was an inverse relationship, suggesting reward deficiency, which would imply that with lower signaling (associated with higher weight), subjects may pursue reward by increasing food intake or by eating more rewarding foods. However, for a wider range of body weights, taking into account a BMI of 19 to 33, a nonlinear model described the data better, such that with a BMI higher than 25, wanting signaling increased progressively with increasing BMI. In effect, this may imply a drive towards a BMI of 25 in normal weight subjects, and reward dissociation in overweight subjects, thereby biasing the latter subjects to maintenance or exacerbation of their overweight (Chapter 2 and 3). To counter this brain based bias, cognitive dietary restraint may be used as a tool by limiting the rewarding value of food in the absence of hunger: In the brain, cognitive dietary restraint modulated the change in signaling from the hungry to the satiated state in the nucleus accumbens (Chapter 2): the higher cognitive dietary restraint scores were, the higher was liking related signaling in the nucleus accumbens pre-meal, and the lower was liking related signaling post-meal. Dietary restraint may thus effectively reduce the rewarding value of food in the absence of hunger, which may then oppose reward dissociation in overweight and obese subjects.

Additional reward dissociation at the central level was indicated during stress i.e. higher cortisol levels. Behavioral liking and wanting related to liking and wanting related signaling during rest conditions pre-meal in respectively the anterior insula and the anterior insula, nucleus accumbens and thalamus. With higher HPA-axis activation, these links were lost pre-meal and new relationships appeared in 
respectively the nucleus accumbens and caudate post-meal. Additionally, during higher HPA-axis activations, higher energy intakes were observed that coincided with reduced wanting task related signaling in the putamen (Chapter 4 and 5). Finally, shifts in macronutrient intakes were predicted by brain specific signaling dependent on the macronutrient, i.e. a downward protein shift related to decreased liking related signaling in the putamen, while a downward carbohydrate shift related to increased wanting related signaling in the hypothalamus. This may represent a central modulation of motivational pathways that can be used to control food choice and energy intake, or which may lead to compensation for extreme macronutrient intakes (Chapter 6).

In conclusion, homeostatic food intake regulation is represented by closely regulated liking and wanting signaling in the brain, which is related to behavioral liking and wanting, BMI and hunger. Liking and wanting represent a partially overlapping network, in which liking is associated with regions implicated in hedonic processes, while wanting was observed in regions implicated in motivation, reward and homeostatic signaling. Thereby, liking and wanting may contribute to reward homeostasis through the determination of the rewarding value of food. Overweight, obesity and stress resulted in reward dissociation, in which the normal link between behavioral liking/wanting and liking/wanting signaling was disturbed, or by a changed relationship between BMI and wanting TRS. These processes ultimately support excessive food intake and altered food choice, yet these effects may be reduced using cognitive dietary restraint as a tool. 
Samenvatting 
Het ontstaan van overgewicht en zwaarlijvigheid wordt toegeschreven aan genetische aanleg, fysiologische factoren, omgevingsfactoren en gedrag. Deze factoren beïnvloeden de energiebalans, d.w.z. de balans tussen energie-inname en energiegebruik. De bijdrage van de energie-inname aan de energiebalans, is tot voor kort voornamelijk gedragsmatig en endocrienologisch onderzocht. Deze studies zijn echter onvolledig: er zijn aanwijzingen dat de belonende waarde van voedselinname een essentiële bijdrage levert aan de energie-inname, en dat dit wellicht niet altijd tot een neutrale energiebalans leidt.

In dit proefschrift worden nieuwe methoden beschreven waarmee de locatie en intensiteit van de belonende waarde van voedsel in het brein wordt bepaald met behulp van functionele kernspinresonantie beeldvorming (functional magnetic resonance imaging, fMRI). De belonende waarde van voedsel is gedefinieerd als een combinatie van twee factoren: Het 'Iusten' en het 'willen'. Het 'lusten' is de hedonische waarde, ofwel 'houden van', en het 'willen' is de motivatie ofwel 'het nu willen eten'. Hoewel het 'lusten' en 'willen' niet volledig onafhankelijk van elkaar zijn, vertegenwoordigen zij niet hetzelfde: Van een product houden wil nog niet zeggen dat men het ook te allen tijde wil consumeren. De huidige resultaten lieten zien dat zowel het 'lusten' als het 'willen' was verminderd na een maaltijd. De verandering van het 'willen' was daarbij echter zeven keer groter dan de verandering van het 'Iusten' (Hoofdstuk 6). Dit verschil wijst erop dat het 'lusten' nauwelijks wordt beïnvloed door honger en verzadiging, terwijl het 'willen' hier juist sterk door wordt beïnvloed. De actieve hersengebieden die bij mensen werden gevonden gedurende de taken waarbij het 'lusten' en 'willen' werden bepaald, kwamen overeen met de hersengebieden die eerder beschreven zij voor 'Iusten' en 'willen' bij knaagdieren. Het 'lusten' was vertegenwoordigd in homeostatische en aan smaakwaarneming gerelateerde gebieden: de insula anterior en posterior, en de cortex cingularis anterior. Het 'willen' was vertegenwoordigd in beloning- en motivatie-gerelateerde gebieden: de caudatus, het putamen en de globus pallidus. Daarnaast was er activering in de thalamus en visuele cortex zowel bij expressie van het 'lusten' als ook bij expressie van het 'willen' (Hoofdstukken 2-4). Dus signalen gerelateerd aan het 'lusten' en 'willen' verlopen via verregaand gescheiden routes in het menselijk brein, en overlappen in specifieke gebieden, zoals de thalamus. Daarnaast bleek dat het signaal in de hypothalamus tijdens de expressie van 'willen' was gerelateerd aan de energie-inname en dat dit signaal in de hypothalamus en het striatum bovendien gerelateerd was a an het lichaamsgewicht (BMI). Bij proefpersonen met een normaal lichaamsgewicht ging een hogere BMI gepaard met een lager signaal en dit suggereerde een gebrek aan beloning (beloningsdeficiëntie). Daarbij gaat men ervan uit dat er meer voedsel, of voedsel met een hogere belonende waarde moet worden geconsumeerd om alsnog voldoende beloning te ervaren. Wanneer echter een groep met meer uiteenlopende gewichten werd bestudeerd, dus een BMI tussen de 19 en $33 \mathrm{~kg} / \mathrm{m}^{2}$, bleek een niet-lineair model beter overeen te komen met de data. Het aan 'willen' gerelateerde signaal nam weer toe met hogere BMI boven de 25. Deze bevindingen wijzen er op dat proefpersonen met een normaal lichaamsgewicht wellicht juist naar een BMI van 25 neigen, en dat dus een hogere gevoeligheid voor beloning leidt tot het zoeken van meer beloning. $\mathrm{Bij}$ proefpersonen met overgewicht en zwaarlijvigheid (BMl>25) vindt er juist weer een dissociatie van de belonende waarde van gedrag (beloningsdissociatie) plaat, en dit 
zou tot het behoud of verergeren van hun overgewicht kunnen leiden (Hoofdstuk 2 en 3).

De aanleg voor een hoger lichaamsgewicht kan worden tegengegaan door het eetgedrag cognitief af te remmen (cognitief geremd eetgedrag) in afwezigheid van honger. In hoofdstuk 2 is beschreven dat dit geremd eetgedrag de verandering van het signaal van het 'lusten' in de nucleus accumbens tussen de gevaste en verzadigde toestand beïnvloedt: Hoe sterker het eetgedrag geremd was, des te hoger het signaal van 'lusten' voor de maaltijd was en des te lager dit na de maaltijd was. Geremd eetgedrag kan dus de belonende waarde van voeding bij afwezigheid van honger wellicht verlagen en daarmee de dissociatie van beloning bij proefpersonen met overgewicht of zwaarlijvigheid voorkomen. In het brein was er tevens een indicatie voor dissociatie van beloning gedurende stress, gekenmerkt door een sterkere activiteit van de hypothalamus-hypofyse-bijnier-as (HPA-as) en een hogere cortisol-concentratie in het bloed. De scores voor het 'lusten' en 'willen' waren in de rustconditie gerelateerd aan de hersenactiviteit tijdens de taken die het 'Iusten' en 'willen' tonen in respectievelijk de insula anterior, nucleus accumbens en thalamus. Bij een sterkere activatie van de HPA-as waren deze relaties niet meer aanwezig en verschenen nieuwe relaties in respectievelijk de nucleus accumbens en caudatus. Tevens vertoonden de proefpersonen een hogere energie-inname die gepaard ging met een lager signaal tijdens de expressie van 'willen' in het putamen (Hoofdstuk 4 en 5). Tenslotte konden de verschuivingen in de macronutriëntinname worden voorspeld aan de hand van macronutriënt-specifieke signaalverschuivingen in het brein: Een verschuiving van een extreem hoge naar een lagere eiwitinname was gerelateerd aan een verminderd 'lusten' signaal in het putamen, terwijl een verschuiving van een extreem hoge naar een lagere koolhydraatinname gerelateerd was aan een verhoogd 'willen' signaal in de hypothalamus. Deze verschillen vertegenwoordigen samen wellicht aanpassingen van motivatie signalen in het brein, die kunnen worden gebruikt om de voedselkeuze en energie-inname te regelen, of die tot compensatiegedrag bij extreme macronutriënt-innamen kunnen leiden (Hoofdstuk 6).

Concluderend kan worden gesteld dat de homeostatische voedselinname-regulatie in het brein is vertegenwoordigd in de vorm van nauwlettend geregelde signalen voor het 'Iusten' en 'willen' van voedsel. Deze signalen zij gerelateerd aan het gedrag, dus hoe hoog het 'lusten' en 'willen' wordt gescoord, en aan de BMI en honger. Het 'lusten' en 'willen' komt tot uiting in een gedeeltelijk overlappend netwerk, waarin het 'lusten' van voedsel is geassocieerd met algemene hedonische gebieden, en waarin het 'willen' is geassocieerd met gebieden die in het algemeen worden gerelateerd aan motivatie, beloning en homeostase. Overgewicht, zwaarlijvigheid en stress leidden tot dissociatie van beloning. Hierbij is de normale relatie tussen de uiting van 'Iusten'/'willen' en de signalen van 'Iusten'/'willen' in het brein verstoord, of is er sprake van een veranderde relatie tussen lichaamsgewicht en de signalen van het 'willen' in het brein. Deze processen zouden uiteindelijk tot excessieve voedselinname en veranderde voedselkeuze kunnen leiden, echter kan cognitief geremde voedselinname deze schadelijke effecten wellicht verminderen. 
Acknowledgements 
En toen waren wij aangekomen bij het meest belangrijke hoofdstuk van het proefschrift, gelet op het aantal lezers dan. Om op dit punt aan te komen is er heel wat werk nodig geweest en hoewel de meeste letters zelf getypt moesten worden, is het vrijwel onhaalbaar zonder de steun van een indrukwekkend aantal mensen! Om te beginnen, wil ik mijn promotores bedanken. Margriet, bedankt voor alles. Voor de snelheid van het nakijken van manuscripten, het enthousiasme en het creëren van gelegenheden om te netwerken op de vele congresbezoeken. Het was een uiterst leerzame en unieke tijd! Rainer, vielen herzlichen Dank für Ihre Vorlesungen, Gespräche und Manuskript-korrekturen, ohne die ich die komplexe Technik "fMRl" nie so richtig hätte verstehen können und für die Einladungen zu Brainvoyagerevents während den HBM Tagungen.

Arie, bedankt voor je input en begeleiding. Jammer dat je er niet langer gebleven bent.

I would like to thank the thesis commission, Prof. dr. ir. R.P. Mensink, Prof. dr. A.A.M. Masclee, Prof. dr. Timothy Moran, Prof. dr. ir. A. Schols and Dr. A. Takashima for reviewing this thesis and for the kind words of appreciation that I received.

Mijn paranimfen Florence en Wieger, wat leuk dat jullie mij willen bijstaan! Met jullie heb ik vaak genoeg gezellig gezeten, gelachen en natuurlijk op zijn tijd ook wel de nodige frustraties gedeeld. Jullie vertegenwoordigen mijn Nijmeegse en Maastrichtse tijd en ik ben blij dit allemaal te met jullie te kunnen delen. Binnenkort ben je zelf ook aan de beurt met de promoties en daarvoor alvast heel veel geduld, geluk en sterkte!

Toen ik in 2007 aan dit traject begon wist ik maar nauwelijks de basis van MRI, laat staat hoe je een scanner bedient, en hoe dan naar de functie van het brein gekeken kan worden met fMRI. Om dit onder de knie te krijgen verdienen een hele reeks mensen alle eer: Sven, bedankt voor de goede instructies bij de scanner, voor alle input, je hulp en de gezelligheid bij de scanner. Zonder jouw inbreng was er weinig van terecht gekomen! Paul, bedankt voor je hulp bij ons eerste fMRI paradigma en voor de enthousiaste en inspirerende 'technische' gesprekken.

Damit wären wir bei Braininnovation angekommen und will ich mich sehr herzlich bedanken bei Armin, der mir viele Stunden seiner wertvollen Zeit geschenkt hat um mir Brainvoyager zu erklären, technische oder Methodologische Schwierigkeiten zu beseitigen, oder auch einfach nur für eine Tasse Kaffee. Bedankt ook Hester, Joost en Pim voor de prettige samenwerking en jullie hulp. When starting up my fMRI analyses, I was pretty much lost. Atsuko and Elia, thank you for your help with the theory of designing fMRI paradigms and analyzing fMRI data. I was clueless when I started and you were two essential teachers!

En dan eindelijk nu eindelijk mijn humbio-(oud)-collega's. Het verhaal begon met Femke, zonder die ik nooit in Maastricht terecht was gekomen. Bedankt voor de tip, ik heb er een leuke tijd aan te danken. Ik denk graag terug aan je 'tea, anyone?'uitnodigingen. Dan de onuitputtelijke Sofie. Vanaf dag één een gezellige collega, die altijd een helpende hand bood. Ik bewonder je geduld om met al die vragen om te gaan ook al heb je zelf een berg aan werk, en dan nóg krijg je toch al je dingen gedaan! $\mathrm{Na}$ het SSIB in portland was het een gezellige tijd met jullie twee in New York, Mama Mia inclusief. Wanneer de dagen erg lang duurden had ik mijn korte pauze-momenten en goed hydratatie-niveau vaak te danken aan Sanne, 'snelle?'. Bovendien, wat zou mijn onderzoek geworden zijn zonder jouw brein? Op een gegeven moment kende je de scanner onderhand beter dan je gewone werkplek. 
Het B1001-team was natuurlijk pas weer helemaal compleet met Mieke die het team weer gezellig kwam versterken. Ik hoefde weinig te doen om je vrolijk te houden, wat ik zei dat was hilarisch, dat trok op niks en dat verstond geen kat! Als ge fMRI maar verstaat ;-). Onze Wageningse collega's bedankt voor de gezellige meetings, Nicolien, Natasja, Annette en Kees. Pleunie en Sanne, onder andere Pittsburgh was echt veel minder gezellig geweest zonder jullie erbij. Ik heb de handdoek van de Pirates-game nog liggen.

De ruimte begint nu op te raken en er blijven nog een hele reeks collega's te noemen, die allemaal een belangrijk steentje hebben bijgedragen aan het geheel: Alberto, Ananda, Andrea, Anneke, Astrid, Eveline, Freeha, Guy, Hanne, Loek, Manuela, Marcel, Margriet, Rick, Stefan, Stijn, en wie ik misschien toch vergeten ben (sorry), allemaal bedankt voor de leuke feesten tijdens congressen, kamers delen in mooie of rare hotels, babbels op de gang en op de kamers aan de uni, in zijn geheel voor een leuke tijd. Claudia, Desiree, Ilona, Jos, Martine en Wendy, bedankt voor jullie ondersteuning! Mijn stagiares Jeroen, Samefko, Sharona en Sonja, leuk dat jullie hebben meegeholpen en ik hoop dat jullie zeker evenveel ervan hebben kunnen leren als ik zelf!

Martijn, Thomas en Erik, met jullie is ons Nijmeegse 'Biologengroepje' compleet, altijd weer leuk om jullie te zien, samen een biertje te pakken (Café Jos misschien), Maastricht onveilig te maken met de carnaval, feesten (vierdaagse of anders) of op vakantie te gaan!

Rita, Grosi, Sabi, Robi, merci vielmol für euri Unterstützig, Gastfründschaft und euer Verständnis wenni widr grad zviel ztue gha ha. Jetzt woni denn fertig bi, han ich denn hoffentlich widr e biz meh Zyt für Bsuech!

Familie en vrienden, bedankt voor jullie interesse, steun en gezelligheid. Ook dat is af en toe hoognodig. My American friends and family, thank you for your hospitality and fun! Without you, my visits to the US would have been much duller!

Mijn ere-paranimf en broer(tje) Pepijn, wat zijn we voortaan toch maar allebei druk he! Maar dat neemt niet weg dat ik heel veel gehad heb aan je input op allerlei vlakken en zonder ons contact had ik echt heel veel moeten missen!

Mam en $\mathrm{Pa}$, zonder jullie had ik dit allemaal natuurlijk al helemaal niet kunnen bereiken. Jullie hebben de basis gelegd waarop ik dit heb kunnen bouwen en jullie stonden altijd klaar ondanks de geografische afstand.

Mijn lieve Claudia, in jou heb ik een liefdevolle, betrouwbare en onvoorwaardelijke dagelijkse steun gevonden. In het begin was het knokken door de afstand tussen Nijmegen en Maastricht. Dat was niet makkelijk en ik ben blij dat het toch goed is gelukt en lk zie onze toekomst blij tegemoet.

Bedankt allen, heel veel geluk en het ga je goed! 
List of Publications 


\section{First Author:}

Born JM, Martens MJ, Rutters F, Lemmens SG, Goebel R, Westerterp-Plantenga MS. High HPA-axis activation disrupts the link between liking and wanting with liking and wanting related brain signaling. Physiol Behav. 2011 Aug 23;105(2):321-324.

Born JM, Lemmens SG, Martens MJ, Formisano E, Goebel R, WesterterpPlantenga MS. Differences between liking and wanting signals in the human brain and relations with cognitive dietary restraint and body mass index. Am J Clin Nutr. 2011 Aug;94(2):392-403.

Born JM, Lemmens SG, Rutters F, Nieuwenhuizen AG, Formisano E, Goebel R, Westerterp-Plantenga MS. Acute stress and food-related reward activation in the brain during food choice during eating in the absence of hunger. Int $\mathrm{J}$ Obes (Lond). 2010 Jan;34(1):172-81.

Born JM, Martens MJ, Lemmens SG, Goebel R, Westerterp-Plantenga MS. A Nonlinear relationship between food choice related brain signaling in the absence of hunger and BMI. Submitted for publication.

Born JM, Martens MJ, Lemmens SG, Goebel R, Westerterp-Plantenga MS. Shifts in protein versus carbohydrate intake differentially affects liking and wanting related brain signaling. Submitten for publication.

\section{Co-author}

Lemmens SG, Born JM, Martens EA, Martens MJ, Westerterp-Plantenga MS. Influence of consumption of a high-protein vs. high-carbohydrate meal on the physiological cortisol and psychological mood response in men and women. PLoS One. $2011 \mathrm{Feb}$ 3;6(2):e16826.

Lemmens SG, Born JM, Rutters F, Schoffelen PF, Wouters L, Westerterp-Plantenga MS. Dietary restraint and control over "wanting" following consumption of "forbidden" food. Obesity (Silver Spring). 2010 Oct;18(10):1926-31.

Lemmens SG, Rutters F, Born JM, Westerterp-Plantenga MS. Stress augments food 'wanting' and energy intake in visceral overweight subjects in the absence of hunger. Physiol Behav. 2011 May 3;103(2):157-63.

Lemmens SG, Martens EA, Born JM, Martens MJ, Westerterp-Plantenga MS. Staggered meal consumption facilitates appetite control without affecting postprandial energy intake. J Nutr. 2011 Mar;141(3):482-8.

Martens MJ, Lemmens SG, Born JM, Westerterp-Plantenga MS. A solid high-protein meal evokes stronger hunger suppression than a liquefied high-protein meal. Obesity (Silver Spring). 2011 Mar;19(3):522-7.

Rutters F, Lemmens SG, Born JM, Bouwman F, Nieuwenhuizen AG, Mariman E, Westerterp-Plantenga MS. Genetic associations with acute stress-related changes 
in eating in the absence of hunger. Patient Educ Couns. 2010 Jun;79(3):367-71.

Lemmens SG, Schoffelen PF, Wouters L, Born JM, Martens MJ, Rutters F, Westerterp-Plantenga MS. Eating what you like induces a stronger decrease of 'wanting' to eat. Physiol Behav. 2009 Sep 7;98(3):318-25.

Martens MJ, Rutters F, Lemmens SG, Born JM, Westerterp-Plantenga MS. Effects of single macronutrients on serum cortisol concentrations in normal weight men. Physiol Behav. 2010 Dec 2;101(5):563-7

Rutters F, Nieuwenhuizen AG, Lemmens SG, Born JM, Westerterp-Plantenga MS. Hypothalamic-pituitary-adrenal (HPA) axis functioning in relation to body fat distribution. Clin Endocrinol (Oxf). 2010 Jun;72(6):738-43.

Rutters F, Nieuwenhuizen AG, Lemmens SG, Born JM, Westerterp-Plantenga MS. Acute stress-related changes in eating in the absence of hunger. Obesity (Silver Spring). 2009 Jan;17(1):72-7.

Rutters F, Nieuwenhuizen AG, Lemmens SG, Born JM, Westerterp-Plantenga MS. Hyperactivity of the HPA axis is related to dietary restraint in normal weight women. Physiol Behav. 2009 Feb 16;96(2):315-9.

\section{Oral presentations}

Born, J.M. Acute stress decreases food related activation in the brain during food choice. NWO, Deurne, The Netherlands, October 2008

Born, J.M.; Nieuwenhuizen, A.G.; Lemmens, S.G.T.; Formisano, E.; Goebel, R.; Westerterp-Plantenga, M.S. Acute stress decreases food reward related activation in the brain. European Congress on Obesity, Amsterdam, The Netherlands, May 2009.

Born, J.; Lemmens, S; Nieuwenhuizen, A; Formisano. E.; Goebel, R., WesterterpPlantenga, M. Acute stress decreases food reward related brain activity. NWO. Deurne, The Netherlands. October 2009.

Born,. J.M. Lemmens, S.G.T.; Martens, M.J.I.; Formisano, E.; Goebel, R.; Westerterp-Plantenga, M.S. Neuronal representation of liking and wanting as a function of hunger and satiety. Society for the Study of Ingestive Behavior (SSIB), Pittsburgh, PA, USA, July 2010. New Invesitgater Travel Award session.

Born,. J.M. Lemmens, S.G.T.; Martens, M.J.I.; Formisano, E.; Goebel, R.; Westerterp-Plantenga, M.S. Neuronal representation of liking and wanting as a function of hunger and satiety. ENP meeting, Lunteren, the Netherlands, May 2011. 


\section{Poster presentations}

Born, J.M.; , Lemmens, S.G.T.; Rutters, F,; Nieuwenhuizen, A.G.; Formisano, E.; Goebel R.; Westerterp-Plantenga, M.S. Acute stress and food reward related activation in the brain during eating in the absence of hunger. Human Brain Mapping. San Francisco, USA. June 2009.

Born, J.; Lemmens, S.; Nieuwenhuizen, A.; Formisano, E.; Goebel, R.; WesterterpPlantenga, M. Acute stress decreases food reward related brain activity. Society for the Study of Ingestive Behavior (SSIB). Portland Oregon, USA. July 2009

Born,. J.M. Lemmens, S.G.T.; Martens, M.J.I.; Formisano, E.; Goebel, R.; Westerterp-Plantenga, M.S. Relation of liking and wanting as a function of hunger. Human Brain Mapping, Barcelona, Spain, June 2010.

Born,. J.M. Lemmens, S.G.T.; Martens, M.J.I.; Formisano, E.; Goebel, R.; Westerterp-Plantenga, M.S. Neuronal representation of liking and wanting as a function of hunger and satiety. International Congress on Obesity, Stockholm, Sweden, July 2010.

Born,. J.M. Lemmens, S.G.T.; Martens, M.J.I.; Formisano, E.; Goebel, R.; Westerterp-Plantenga, M.S. Neuronal representation of liking and wanting as a function of hunger and satiety. Society for Neuroscience annual meeting, San Diego, USA, November 2010.

\section{Software}

Brainvoyager Analysis Predictor Tool (BVA-Predictor tool), 2008-2011.

http://support.brainvoyager.com/available-tools/53-user-developed-tools/238brainvoyager-analysis-predictor-tool.html. 
About the Author 
Jurriaan Born was born on May 19th 1978 in Herdecke, Germany. He attended the Freie Waldorfschule Frankfurt, Germany, where he graduated in 1998. While working in IT support for different companies, he moved to the Netherlands. To qualify for studying Biology, he decided to obtain a chemistry diploma at the James Boswell Institute, Utrecht, after which he started his study medical biology at the Radboud University Nijmegen, the Netherlands, for which he received his masters degree in 2007. In May of 2007, Jurriaan continued to Maastricht University as a PhD student on the Top Institute Food and Nutrition (TIFN) project "Effects of physical chemical properties of food on sensory satiety, metabolic satiety, reward and food intake regulation", located at the department of Human Biology, Maastricht University. While executing his research, he expanded his hobby of programming, which resulted in publishing a software tool (Brainvoyager Analysis Predictor Tool), which is used to assist in creating mathematical predictor models for neuro-imaging data, which is freely available to fellow researchers. Additionally, he authored a questionnaire tool, which is now used by many of his former colleagues. The research performed within this project, under supervision of Prof. dr. Margriet Westerterp-Plantenga and Prof. dr. Rainer Goebel, resulted in this thesis entitled "Representation of the rewarding value of food in the human brain - Effects of dietary restraint, body weight, stress, and shifts in macronutrient intake" and was approved in September of 2011. During his PhD project, Jurriaan presented several abstracts at international conferences and was awarded a New Investigator Travel Award from the Society for the Study of Ingestive Behavior, Pittsburgh, PA, USA in June of 2010.

Currently, Jurriaan is working as a clinical study team consultant for the 'Clinbrowser' software project, a clinical study review tool, at Janssen Pharmaceuticals in Beerse, Belgium. 
